

\title{
On 2-dimensional 2-adic Galois representations of local and global fields
}

\author{
Vytautas Paškūnas
}

We describe the generic blocks in the category of smooth locally admissible mod-2 representations of $\mathrm{GL}_{2}\left(\mathbb{Q}_{2}\right)$. As an application we obtain new cases of the Breuil-Mézard and Fontaine-Mazur conjectures for 2-dimensional 2-adic Galois representations.

1. Introduction 1301

1A. Outline of the paper 1305

2. Local part 1307

2A. Capture 1307

2B. The image of Colmez's Montreal functor 1310

2C. The Breuil-Mézard conjecture 1323

3. Global part 1330

3A. Quaternionic modular forms 1330

3B. Residual Galois representation 1332

3C. Patching 1334

3D. Small weights 1343

3E. Computing Hilbert-Samuel multiplicity 1346

3F. Modularity lifting 1353

Acknowledgements 1355

References 1355

\section{Introduction}

Let $p$ be a prime and let $L$ be a finite extension of $\mathbb{Q}_{p}$ with the ring of integers $\mathcal{O}$ and uniformizer $\varpi$. We prove the following modularity lifting theorem.

Keywords: $p$-adic Langlands, Fontaine-Mazur, modularity lifting. 
Theorem 1.1. Assume that $p=2$. Let $F$ be a totally real field where 2 is totally split, let $S$ be a finite set of places of $F$ containing all the places above 2 and all the infinite places and let

$$
\rho: G_{F, S} \rightarrow \mathrm{GL}_{2}(\mathcal{O})
$$

be a continuous representation of the Galois group of the maximal extension of $F$ unramified outside S. Suppose:

(i) $\bar{\rho}: G_{F, S} \stackrel{\rho}{\rightarrow} \mathrm{GL}_{2}(\mathcal{O}) \rightarrow \mathrm{GL}_{2}(k)$ is modular with nonsolvable image.

(ii) If $v \mid 2$ then $\left.\rho\right|_{G_{F_{v}}}$ is potentially semistable with distinct Hodge-Tate weights.

(iii) $\operatorname{det} \rho$ is totally odd.

(iv) If $v \mid 2$ then $\left.\bar{\rho}\right|_{G_{F_{v}}} ¥\left(\begin{array}{ll}\chi & * \\ 0 & \chi\end{array}\right)$ for any character $\chi: G_{F_{v}} \rightarrow k^{\times}$.

Then $\rho$ is modular.

Kisin [2009a] and Emerton [2011] have proved an analogous theorem for $p>2$. Our proof follows the strategy of Kisin. We patch automorphic forms on definite quaternion algebras and deduce the theorem from a weak form of the Breuil-Mézard conjecture, which we prove for all $p$ under some technical assumptions on the residual representation of $G_{\mathbb{Q}_{p}}$ (see Theorems 2.34 and 2.37) which force us to assume (iv) in the theorem.

The Breuil-Mézard conjecture is proved by employing a formalism developed in [Paškūnas 2015b], where an analogous result is proved under the assumption that $p \geq 5$ and the residual representation has scalar endomorphisms. We can prove the result for primes 2 and 3 by better understanding the smooth representation theory of $G:=\mathrm{GL}_{2}\left(\mathbb{Q}_{p}\right)$ in characteristic $p$ : in the local part of the paper we extend the results of [Paškūnas 2013] to the generic blocks, when $p$ is 2 and 3, which we will now describe.

Let $\operatorname{Mod}_{G}^{\mathrm{sm}}(\mathcal{O})$ be the category of smooth $G$-representation on $\mathcal{O}$-torsion modules. We fix a continuous character $\psi: \mathbb{Q}_{p}^{\times} \rightarrow \mathcal{O}^{\times}$and let $\operatorname{Mod}_{G, \psi}^{1 . a d m}(\mathcal{O})$ be the full subcategory of $\operatorname{Mod}_{G}^{\mathrm{sm}}(\mathcal{O})$, consisting of representations on which the center of $G$ acts by the character $\psi$ and which are equal to the union of their admissible subrepresentations. The categories $\operatorname{Mod}_{G}^{\mathrm{sm}}(\mathcal{O})$ and $\operatorname{Mod}_{G, \psi}^{\mathrm{ladm}}(\mathcal{O})$ are abelian; see [Emerton 2010a, Proposition 2.2.18]. A finitely generated smooth admissible representation of $G$ with a central character is of finite length by Theorem 2.3.8 of [Emerton 2010a]. This makes $\operatorname{Mod}_{G, \psi}^{1 . \mathrm{adm}}(\mathcal{O})$ into a locally finite category. Gabriel [1962] has proved that a locally finite category decomposes into a direct product of indecomposable subcategories as follows.

Let $\operatorname{Irr}_{G}^{\text {adm }}$ be the set of irreducible representations in $\operatorname{Mod}_{G, \psi}^{1 . \operatorname{adm}}(\mathcal{O})$. We define an equivalence relation $\sim$ on $\operatorname{Irr}_{G}^{\text {adm }}$ by writing $\pi \sim \tau$ if there exists a sequence $\pi=\pi_{1}, \pi_{2}, \ldots, \pi_{n}=\tau$ in $\operatorname{Irr}_{G}^{\text {adm }}$ such that for each $i$ one of the following holds: 
(1) $\pi_{i} \cong \pi_{i+1}$; (2) $\operatorname{Ext}_{G}^{1}\left(\pi_{i}, \pi_{i+1}\right) \neq 0$; (3) $\operatorname{Ext}_{G}^{1}\left(\pi_{i+1}, \pi_{i}\right) \neq 0$. We have a canonical decomposition

$$
\operatorname{Mod}_{G, \psi}^{\operatorname{l.adm}}(\mathcal{O}) \cong \prod_{\mathfrak{B} \in \operatorname{Irr}_{G}^{\text {adm }} / \sim} \operatorname{Mod}_{G, \psi}^{1 . \operatorname{adm}}(\mathcal{O})[\mathfrak{B}]
$$

where $\operatorname{Mod}_{G, \psi}^{1 . a d m}(\mathcal{O})[\mathfrak{B}]$ is the full subcategory of $\operatorname{Mod}_{G, \psi}^{1 . a d m}(\mathcal{O})$ consisting of representations with all irreducible subquotients in $\mathfrak{B}$. A block is an equivalence class of $\sim$.

For a block $\mathfrak{B}$ let $\pi_{\mathfrak{B}}=\bigoplus_{\pi \in \mathfrak{B}} \pi$, let $\pi_{\mathfrak{B}} \hookrightarrow J_{\mathfrak{B}}$ be an injective envelope of $\pi_{\mathfrak{B}}$ and let $E_{\mathfrak{B}}:=\operatorname{End}_{G}\left(J_{\mathfrak{B}}\right)$. Then $J_{\mathfrak{B}}$ is an injective generator for $\operatorname{Mod}_{G, \psi}^{1 . \operatorname{adm}}(\mathcal{O})[\mathfrak{B}], E_{\mathfrak{B}}$ is a pseudocompact ring and the functor $\kappa \mapsto \operatorname{Hom}_{G}\left(\kappa, J_{\mathfrak{B}}\right)$ induces an antiequivalence of categories between $\operatorname{Mod}_{G, \psi}^{1 . \mathrm{adm}}(\mathcal{O})[\mathfrak{B}]$ and the category of right pseudocompact $E_{\mathfrak{B}}$-modules. The inverse functor is given by $\mathrm{m} \mapsto\left(\mathrm{m} \widehat{\otimes}_{E_{\mathfrak{B}}} J_{\mathfrak{B}}^{\vee}\right)^{\vee}$, where $\vee$ denotes the Pontryagin dual; see [Gabriel 1962, Chapitre IV, §4]. The main result of [Paškūnas 2013] computes the rings $E_{\mathfrak{B}}$ for each block $\mathfrak{B}$ and describes the Galois representation of $G_{\mathbb{Q}_{p}}$ obtained by applying the Colmez's functor to $J_{\mathfrak{B}}$ under the assumption $p \geq 5$ or $p \geq 3$, depending on the block $\mathfrak{B}$.

If $\pi \in \operatorname{Irr}_{G}^{\mathrm{adm}}$ then one may show that, after extending scalars, $\pi$ is isomorphic to a finite direct sum of absolutely irreducible representations of $G$. It has been proved in [Paškūnas 2014] that the blocks containing an absolutely irreducible representation are given by

(i) $\mathfrak{B}=\{\pi\}$ with $\pi$ supersingular;

(ii) $\mathfrak{B}=\left\{\left(\operatorname{Ind}_{B}^{G} \chi_{1} \otimes \chi_{2} \omega^{-1}\right)_{\mathrm{sm}},\left(\operatorname{Ind}_{B}^{G} \chi_{2} \otimes \chi_{1} \omega^{-1}\right)_{\mathrm{sm}}\right\}$ with $\chi_{2} \chi_{1}^{-1} \neq \omega^{ \pm 1}, \mathbf{1}$;

(iii) $p>2$ and $\mathfrak{B}=\left\{\left(\operatorname{Ind}_{B}^{G} \chi \otimes \chi \omega^{-1}\right)_{\text {sm }}\right\}$;

(iv) $p=2$ and $\mathfrak{B}=\{\mathbf{1}, \mathrm{Sp}\} \otimes \chi \circ$ det;

(v) $p \geq 5$ and $\mathfrak{B}=\left\{\mathbf{1}, \mathrm{Sp},\left(\operatorname{Ind}_{B}^{G} \omega \otimes \omega^{-1}\right)_{\mathrm{sm}}\right\} \otimes \chi \circ \mathrm{det}$;

(vi) $p=3$ and $\mathfrak{B}=\{\mathbf{1}, \operatorname{Sp}, \omega \circ \operatorname{det}, \operatorname{Sp} \otimes \omega \circ \operatorname{det}\} \otimes \chi \circ \operatorname{det}$;

where $\chi, \chi_{1}, \chi_{2}: \mathbb{Q}_{p}^{\times} \rightarrow k^{\times}$are smooth characters, $\omega: \mathbb{Q}_{p}^{\times} \rightarrow k^{\times}$is the character $\omega(x)=x|x|(\bmod \varpi)$ and we view $\chi_{1} \otimes \chi_{2}$ as a character of the subgroup of upper-triangular matrices $B$ in $G$ which sends $\left(\begin{array}{ll}a & b \\ 0 & d\end{array}\right)$ to $\chi_{1}(a) \chi_{2}(d)$. An absolutely irreducible representation $\pi$ is supersingular if it is not a subquotient of a principal series representation (they have been classified by Breuil [2003a]) and Sp denotes the Steinberg representation.

To each block above one may attach a semisimple 2-dimensional $k$-representation $\bar{\rho}^{\text {ss }}$ of $G_{\mathbb{Q}_{p}}$ : in case (i) $\bar{\rho}^{\text {ss }}$ is absolutely irreducible, and such that Colmez's functor $\boldsymbol{V}$ (see Section 2B1) maps $\pi$ to $\bar{\rho}^{\text {ss }}$; in case (ii) $\bar{\rho}^{\text {ss }}=\chi_{1} \oplus \chi_{2}$; in cases (iii) and (iv) $\bar{\rho}^{\text {ss }}=\chi \oplus \chi$; in cases (v) and (vi) $\bar{\rho}^{\text {ss }}=\chi \oplus \chi \omega$, where we consider characters of $G_{\mathbb{Q}_{p}}$ as characters of $\mathbb{Q}_{p}^{\times}$via local class field theory, normalized so that uniformizers 
correspond to geometric Frobenii. We note that the determinant of $\bar{\rho}^{\text {ss }}$ is equal to $\psi \varepsilon$ modulo $\varpi$, where $\varepsilon$ is the $p$-adic cyclotomic character and $\omega$ is its reduction modulo $\varpi$.

Theorem 1.2. If $\mathfrak{B}=\{\pi\}$ with $\pi$ supersingular (so that $\bar{\rho}^{\mathrm{ss}}$ is irreducible) then $E_{\mathfrak{B}}$ is naturally isomorphic to the quotient of the universal deformation ring of $\bar{\rho}^{\mathrm{ss}}$ parametrizing deformations with determinant $\psi \varepsilon$, and $\boldsymbol{V}\left(J_{\mathfrak{B}}\right)^{\vee}(\psi \varepsilon)$ is a tautological deformation of $\bar{\rho}^{\mathrm{ss}}$ to $E_{\mathfrak{B}}$.

We also obtain an analogous result for blocks in (ii); see Theorem 2.23. Let $R^{\mathrm{ps}}$ be the deformation ring parametrizing all the 2-dimensional determinants, in the sense of [Chenevier 2014], lifting $\left(\operatorname{tr} \bar{\rho}^{-\mathrm{ss}}\right.$, det $\left.\bar{\rho}^{-\mathrm{ss}}\right)$, and let $R^{\mathrm{ps}, \psi}$ be the quotient of $R^{\mathrm{ps}}$ parametrizing those which have determinant $\psi \varepsilon$.

Theorem 1.3. Assume that the block $\mathfrak{B}$ is given by (i) or (ii) above. Then the center of the category $\operatorname{Mod}_{G, \psi}^{1 . \mathrm{adm}}(\mathcal{O})[\mathfrak{B}]$ is naturally isomorphic to $R^{\mathrm{ps}, \psi}$.

We view this theorem as an analogue of the Bernstein center for this category. Theorems 1.2 and 1.3 are new if $p=2$ and if $p=3$ and $\mathfrak{B}=\{\pi\}$ with $\pi$ supersingular. Together with the results of [Paškūnas 2013] this covers all the blocks except for those in (iv) and (vi) above.

One also has a decomposition similar to (1) for the category $\operatorname{Ban}_{G, \psi}^{\mathrm{adm}}(L)$ of admissible unitary $L$-Banach space representations of $G$ on which the center of $G$ acts by $\psi$; see Section 2B4. An admissible unitary $L$-Banach space representation $\Pi$ lies in $\operatorname{Ban}_{G, \psi}^{\mathrm{adm}}(L)[\mathfrak{B}]$ if and only if all the irreducible subquotients of the reduction modulo $\varpi$ of a unit ball in $\Pi$ modulo $\varpi$ lie in $\mathfrak{B}$. An irreducible $\Pi$ is ordinary if it is a subquotient of a unitary parabolic induction of a unitary character. Otherwise it is called nonordinary.

Corollary 1.4. Assume that the block $\mathfrak{B}$ is given by (i) or (ii) above. Colmez's Montreal functor $\Pi \mapsto \check{V}(\Pi)$ induces a bijection between the isomorphism classes of

- absolutely irreducible nonordinary $\Pi \in \operatorname{Ban}_{G, \psi}^{\mathrm{adm}}(L)[\mathfrak{B}]$;

- absolutely irreducible $\tilde{\rho}: G_{\mathbb{Q}_{p}} \rightarrow \mathrm{GL}_{2}(L)$ such that $\operatorname{det} \tilde{\rho}=\psi \varepsilon$ and the semisimplification of the reduction modulo $\varpi$ of a $G_{\mathbb{Q}_{p}}$-invariant $\mathcal{O}$-lattice in $\tilde{\rho}$ is isomorphic to $\bar{\rho}^{\mathrm{ss}}$.

A stronger result, avoiding the assumption on $\mathfrak{B}$, is proved in [Colmez et al. 2014]. However, our proof of Corollary 1.4 avoids the hard $p$-adic functional analysis, which is used to construct representations of $\mathrm{GL}_{2}\left(\mathbb{Q}_{p}\right)$ out of 2-dimensional representations of $G_{\mathbb{Q}_{p}}$ via the theory of $(\varphi, \Gamma)$-modules by Colmez [2010], which plays the key role in [Colmez et al. 2014].

It might be possible, given the global part of this paper, and the results of [Paškūnas 2015a], where various deformation rings are computed, when $p=2$, 
to prove Theorem 1.1 by repeating the arguments of Kisin [2009a]. We have not checked this. However, our original goal was to prove Theorems 1.2 and 1.3; Theorem 1.1 came out as a bonus at the end.

1A. Outline of the paper. The paper has two largely independent parts: a local one and a global one. We will review each of them individually by carefully explaining which arguments are new.

1A1. Local part. For concreteness, assume that $\mathfrak{B}=\{\pi\}$ with $\pi$ supersingular. Let $\bar{\rho}=\boldsymbol{V}(\pi)$, let $R_{\bar{\rho}}$ be the universal deformation ring of $\bar{\rho}$ and let $R_{\bar{\rho}}^{\psi}$ be the quotient of $R_{\bar{\rho}}$ parametrizing deformations with determinant $\psi \varepsilon$. We follow the strategy outlined in [Paškūnas 2013, §5.8]. We show that $J_{\mathfrak{B}}^{\vee}$ is the universal deformation of $\pi^{\vee}$ and $E_{\mathfrak{B}}$ is the universal deformation ring by verifying that hypotheses (H0)-(H5), made in Section 3 of [Paškūnas 2013], hold. In Section 3.3 of the same work we developed a criterion to check that the ring $E_{\mathfrak{B}}$ is commutative. To apply this criterion, one needs the ring $R_{\bar{\rho}}^{\psi}$ to be formally smooth and to control the image of some $\mathrm{Ext}^{1}$-group in some $\mathrm{Ext}^{2}$-group. The first condition does not hold if $p=2$ and if $p=3$ and $\bar{\rho} \cong \bar{\rho} \otimes \omega$. Even if $p=3$ and $\bar{\rho} ¥ \bar{\rho} \otimes \omega$, so that the ring is formally smooth, to check the second condition is a computational nightmare. In [Colmez et al. 2014] we found a different characteristic-0 argument to get around this. The key input is the result of [Berger and Breuil 2010] which says that if a locally algebraic principal series representation admits a $G$-invariant norm, then its completion is irreducible, and $\pi$ occurs in the reduction modulo $\varpi$ with multiplicity one. We deduce from [Colmez et al. 2014, Corollary 2.22] that the ring $E_{\mathfrak{B}}$ is commutative. The argument of Kisin [2010] shows that $\boldsymbol{V}\left(J_{\mathfrak{B}}\right)^{\vee}(\psi \varepsilon)$ is a deformation of $\bar{\rho}$ to $E_{\mathfrak{B}}$ and we have surjections $R_{\bar{\rho}} \rightarrow E_{\mathfrak{B}} \rightarrow R_{\bar{\rho}}^{\psi}$.

To prove Theorem 1.2 we have to show that the surjection $\varphi: E_{\mathfrak{B}} \rightarrow R_{\bar{\rho}}^{\psi}$ is an isomorphism. The proof of this claim is new and is carried out in Section 2B3. Corollary 1.4 is then a formal consequence of this isomorphism. If $p \geq 5$ then $R_{\bar{\rho}}^{\psi}$ is formally smooth and the claim is proved by a calculation on tangent spaces in [Paškūnas 2013]. This does not hold if $p=2$ or $p=3$ and $\bar{\rho} \cong \bar{\rho} \otimes \omega$. We also note that even if we admit the main result of [Colmez et al. 2014] (which we don't), we would only get that $\varphi$ induces a bijection on maximal spectra of the generic fibers of the rings. From this one could deduce that the map induces an isomorphism between the maximal reduced quotient of $E_{\mathfrak{B}}$ and $R_{\bar{\rho}}^{\psi}$, and it is not at all clear that $E_{\mathfrak{B}}$ is reduced. However, by using techniques of [Paškūnas 2015b] we can show that certain quotients $E_{\mathfrak{B}} / \mathfrak{a}$ are reduced and identify them with crystabeline deformation rings of $\bar{\rho}$ via $\varphi$. Again the argument uses the results of [Berger and Breuil 2010] in a crucial way. Further, we show that the intersection of all such ideals in $E_{\mathfrak{B}}$ is zero, which allows us to conclude the proof. A similar argument using density appears in [Colmez et al. 2014, §2.4], however we have to work a bit 
more here, because we fix a central character; see Section 2A. Theorem 1.2 implies immediately that $\operatorname{det} \check{\boldsymbol{V}}(\Pi)=\psi \varepsilon$ for all $\Pi \in \operatorname{Ban}_{G, \psi}^{\text {adm }}(L)[\mathfrak{B}]$. This is proved directly in [Colmez et al. 2014] without any restriction on $\mathfrak{B}$, and is the most technical part of that paper.

Once we have Theorem 1.2, the Breuil-Mézard conjecture is proved the same way as in [Paškūnas 2015b]; see Section $2 \mathrm{C}$. If $\mathfrak{B}$ is the block containing two generic principal series representations, so that $\bar{\rho}^{\mathrm{ss}}=\chi_{1} \oplus \chi_{2}$, with $\chi_{1} \chi_{2}^{-1} \neq \mathbf{1}, \omega^{ \pm 1}$, then we prove the Breuil-Mézard conjecture for both nonsplit extensions $\left(\begin{array}{cc}\chi_{1} & * \\ 0 & \chi_{2}\end{array}\right)$ and $\left(\begin{array}{cc}\chi_{1} & 0 \\ * & \chi_{2}\end{array}\right)$ and deduce the conjecture in the split case in a companion paper [Paškūnas 2015a], following an idea of $\mathrm{Hu}$ and Tan [2015]. We formulate and prove the Breuil-Mézard conjecture in the language of cycles, as introduced by Emerton and Gee [2014]. All our arguments are local, except that if the inertial type extends to an irreducible representation of the Weil group $W_{\mathbb{Q}_{p}}$ of $\mathbb{Q}_{p}$, the description of locally algebraic vectors in the Banach space representations relies on a global input of Emerton [2011, §7.4]. Dospinescu's results [2015] on locally algebraic vectors in extensions of Banach space representations of $G$ are also crucial in this case.

1A2. Global part. As already explained, an analogue of Theorem 1.1 has been proved by Kisin if $p>2$. Moreover, if $p=2$ and $\left.\rho\right|_{G_{F_{v}}}$ is semistable with HodgeTate weights $(0,1)$ for all $v \mid 2$, then the theorem has been proved by Khare and Wintenberger [2009b] and Kisin [2009b] in their work on Serre's conjecture. We use their results as an input in our proof.

The strategy of the proof is the same as in [Kisin 2009a]. By base change arguments, which are the same as in [Khare and Wintenberger 2009b; Kisin 2009b; 2009c] (see Section 3F) we reduce ourselves to a situation where the ramification of $\rho$ and $\bar{\rho}$ outside 2 is minimal and $\bar{\rho}$ comes from an automorphic form on a definite quaternion algebra. We patch automorphic forms on definite quaternion algebras and deduce the theorem from a weak form of the Breuil-Mézard conjecture, which is proved in the local part of the paper. Assumption (iv) in Theorem 1.1 comes from the local part of the paper.

Let us explain some differences with [Kisin 2009a]. If $p>2$ then the patched ring is formally smooth over a completed tensor product of local deformation rings. This implies that the patched ring is reduced, equidimensional and $\mathcal{O}$-flat and that its Hilbert-Samuel multiplicity is equal to the product of Hilbert-Samuel multiplicities of the local deformation rings. For $p=2$ we modify the patching argument used in [Kisin 2009a] following [Khare and Wintenberger 2009b]. This gives us two patched rings, and the passage between them and the completed tensor product of local rings is not as straightforward as before. To overcome this we use an idea which appears in errata to [Kisin 2009a] published in [Gee and Kisin 2014]. If $\rho_{f}$ is a Galois representation associated to a Hilbert modular form lifting $\bar{\rho}$ and $v$ is a place of $F$ above $p$, then one knows from [Blasius 2006; Katz and Messing 
1974; Saito 2009] that the Weil-Deligne representation associated to $\left.\rho\right|_{G_{F_{v}}}$ is pure. Kisin shows that this implies that the point on the generic fiber of the potentially semistable deformation ring, defined by $\left.\rho_{f}\right|_{G_{F_{v}}}$, cannot lie on the intersection of two irreducible components, and hence is regular. Using this we show that the localization of patched rings at the prime ideal defined by $\rho_{f}$ is regular, and we are in a position to use the Auslander-Buchsbaum theorem; see Lemma 3.14 and Proposition 3.17. As explained in [Gee and Kisin 2014], this observation enables us to deal with cases when the patched module is not generically free of rank 1 over the patched ring, which was the case in the original paper [Kisin 2009a]. In particular, we don't add any Hecke operators at places above 2 and we don't use [Darmon et al. 1997, Lemma 4.11].

As a part of his proof, Kisin uses the description by Gee [2011] of Serre weights for $\bar{\rho}$, which is available only for $p>2$. We determine Serre weights for $\bar{\rho}$ when $p=2$ in Section 3D under assumption (iv) of Theorem 1.1. As in [Gee 2011] the main input is a modularity lifting theorem, which in our case is the theorem proved by Khare and Wintenberger [2009b] and Kisin [2009b]. We do this by a characteristic-0 argument, where Gee argues in characteristic $p$; see Section 3D.

The modularity lifting theorems for $p=2$ proved by Kisin [2009b], and more recently by Thorne [2016], do not require 2 to split completely in the totally real field $F$, but they put a more restrictive hypothesis on $\left.\rho\right|_{G_{F}}$ for $v \mid 2$. Kisin assumes that $\left.\rho\right|_{G_{F_{v}}}$ for all $v \mid 2$ is potentially crystalline with Hodge-Tate weights equal to $(0,1)$ for every embedding $F_{v} \hookrightarrow \overline{\mathbb{Q}}_{2}$ and $F_{v}=\mathbb{Q}_{2}$ if $\left.\rho\right|_{G_{F_{v}}}$ is ordinary. Thorne removes this last assumption, but requires instead that $\left.\bar{\rho}\right|_{G_{F_{v}}}$ be nontrivial for at least one $v \mid \infty$. We need 2 to split completely in $F$ in order to apply the results on the $p$-adic Langlands correspondence, which is currently available only for $\mathrm{GL}_{2}\left(\mathbb{Q}_{p}\right)$.

\section{Local part}

2A. Capture. Let $K$ be a profinite group with an open pro- $p$ group. Let $\mathcal{O} \llbracket K \rrbracket$ be the completed group algebra, and let $\operatorname{Mod}_{K}^{\text {pro }}(\mathcal{O})$ be the category of compact lineartopological $\mathcal{O} \llbracket K \rrbracket$-modules. Let $\psi: Z(K) \rightarrow \mathcal{O}^{\times}$be a continuous character. We let $\operatorname{Mod}_{K, \psi}^{\text {pro }}(\mathcal{O})$ be the full subcategory of $\operatorname{Mod}_{K}^{\text {pro }}(\mathcal{O})$ such that $M \in \operatorname{Mod}_{K}^{\text {pro }}(\mathcal{O})$ lies in $\operatorname{Mod}_{K, \psi}^{\text {pro }}(\mathcal{O})$ if and only if $Z(K)$ acts on $M$ via $\psi^{-1}$. Let $\left\{V_{i}\right\}_{i \in I}$ be a family of continuous representations of $K$ on finite-dimensional $L$-vector spaces, and let $M \in \operatorname{Mod}_{K}^{\text {pro }}(\mathcal{O})$.

Definition 2.1. We say that $\left\{V_{i}\right\}_{i \in I}$ captures $M$ if the smallest quotient $M \rightarrow Q$ such that $\operatorname{Hom}_{\mathcal{O} \llbracket K \rrbracket}^{\text {cont }}\left(Q, V_{i}^{*}\right) \cong \operatorname{Hom}_{\mathcal{O} \llbracket K \rrbracket}^{\text {cont }}\left(M, V_{i}^{*}\right)$ for all $i \in I$ is equal to $M$.

We let $c:=\left(\begin{array}{rr}-1 & 0 \\ 0 & -1\end{array}\right)$ and note that the center of $\operatorname{SL}_{2}\left(\mathbb{Z}_{p}\right)$ is equal to $\{1, c\}$.

Lemma 2.2. If $K=\mathrm{SL}_{2}\left(\mathbb{Z}_{p}\right)$ then $\mathcal{O} \llbracket K \rrbracket /(c-1)$ and $\mathcal{O} \llbracket K \rrbracket /(c+1)$ are $\mathcal{O}$-torsionfree. 
Proof. If $K_{n}$ is an open normal subgroup of $K$ such that the image of $c$ in $K / K_{n}$ is nontrivial, then $\mathcal{O}\left[K / K_{n}\right]$ is a free $\mathcal{O}[Z]$-module, where $Z$ is the center of $K$. This implies that $\mathcal{O}\left[K / K_{n}\right] /(c \pm 1)$ is a free $\mathcal{O}$-module and by passing to the limit we obtain the assertion.

Lemma 2.3. Let $K=\mathrm{SL}_{2}\left(\mathbb{Z}_{p}\right)$, let $Z$ be the center of $K$ and let $\left\{V_{i}\right\}_{i \in I}$ be a family which captures $\mathcal{O} \llbracket K \rrbracket$ such that each $V_{i}$ has a central character. Let $I^{+}$and $I^{-}$ be subsets of I consisting of $i$ such that $c$ acts on $V_{i}$ by 1 and by -1 , respectively. Let $\psi: Z \rightarrow L^{\times}$be a character. If $\psi(c)=1$ then $I^{+}$captures every projective object in $\operatorname{Mod}_{K, \psi}^{\text {pro }}(\mathcal{O})$. If $\psi(c)=-1$ then $I^{-}$captures every projective object in $\operatorname{Mod}_{K, \psi}^{\text {pro }}(\mathcal{O})$.

Proof. If $M \in \operatorname{Mod}_{K}^{\text {pro }}(\mathcal{O})$ is $\mathcal{O}$-torsion-free then $I$ captures $M$ if and only if the image of the evaluation map $\bigoplus_{i \in I} V_{i} \otimes \operatorname{Hom}_{K}\left(V_{i}, \Pi\right) \rightarrow \Pi$ is dense, where $\Pi=\operatorname{Hom}_{\mathcal{O}}^{\text {cont }}(M, L)$ is the Banach space representation of $K$ with the topology induced by the supremum norm [Colmez et al. 2014, Lemma 2.10]. Let $\Pi=\operatorname{Hom}_{\mathcal{O}}^{\text {cont }}(\mathcal{O} \llbracket K \rrbracket, L)$ and $\Pi^{ \pm}:=\operatorname{Hom}_{\mathcal{O}}^{\text {cont }}(\mathcal{O} \llbracket K \rrbracket /(c \pm 1), L)$. Since $\Pi=$ $\Pi^{+} \oplus \Pi^{-}$, and $\left\{V_{i}\right\}$ captures $\mathcal{O} \llbracket K \rrbracket$, we deduce that the image of the evaluation map $\bigoplus_{i \in I} V_{i} \otimes \operatorname{Hom}_{K}\left(V_{i}, \Pi^{ \pm}\right) \rightarrow \Pi^{ \pm}$is dense. If $i \in I^{+}$then $c$ acts trivially on $V_{i}$ and so $\operatorname{Hom}_{K}\left(V_{i}, \Pi^{-}\right)=0$. This implies the image of $\bigoplus_{i \in I^{+}} V_{i} \otimes \operatorname{Hom}_{K}\left(V_{i}, \Pi^{+}\right) \rightarrow \Pi^{+}$ is dense. Using Lemma 2.2 we deduce that $I^{+}$captures $\mathcal{O} \llbracket K \rrbracket /(c-1)$. A similar argument shows that $I^{-}$captures $\mathcal{O} \llbracket K \rrbracket /(c+1)$. Every projective object in $\operatorname{Mod}_{K, \psi}^{\text {pro }}(\mathcal{O})$ can be realized as a direct summand of a product of some copies of $\mathcal{O} \llbracket K \rrbracket /(c-\psi(c))$, which implies the assertion; see the proof of [Colmez et al. 2014, Lemma 2.11].

Lemma 2.4. Let $K=\mathrm{SL}_{2}\left(\mathbb{Z}_{p}\right)$, and let $Z$ be the center of $K, \psi: Z \rightarrow L^{\times}$a character and $V$ a continuous representation of $K$ on a finite-dimensional $L$-vector space with a central character $\psi_{V}$. If $\psi(c)=\psi_{V}(c)$ then $\left\{V \otimes \operatorname{Sym}^{2 a} L^{2}\right\}_{a \in \mathbb{N}}$ captures every projective object in $\operatorname{Mod}_{K, \psi}^{\mathrm{pro}}(\mathcal{O})$; if $\psi(c)=-\psi_{V}(c)$ then $\left\{V \otimes \operatorname{Sym}^{2 a+1} L^{2}\right\}_{a \in \mathbb{N}}$ captures every projective object in $\operatorname{Mod}_{K, \psi}^{\mathrm{pro}}(\mathcal{O})$.

Proof. Proposition 2.12 in [Colmez et al. 2014] implies that $\left\{\operatorname{Sym}^{a} L^{2}\right\}_{a \in \mathbb{N}}$ captures $\mathcal{O} \llbracket K \rrbracket$. We leave it as an exercise for the reader to check that this implies that $\left\{V \otimes \operatorname{Sym}^{a} L^{2}\right\}_{a \in \mathbb{N}}$ also captures $\mathcal{O} \llbracket K \rrbracket$. The assertion follows from Lemma 2.3.

Lemma 2.5. Let $M \in \operatorname{Mod}_{\mathrm{GL}_{2}\left(\mathbb{Z}_{p}\right), \psi}^{\mathrm{pro}}(\mathcal{O})$ and let $V$ be a continuous representation of $K$ on a finite-dimensional L-vector space with a central character $\psi$. Then

$$
\bigcap_{\phi} \operatorname{Ker} \phi=\bigcap_{\xi, \eta} \operatorname{Ker} \xi
$$

where the first intersection is taken over all $\phi \in \operatorname{Hom}_{\mathcal{O}\left[\mathrm{SL}_{2}\left(\mathbb{Z}_{p}\right) \|\right.}^{\mathrm{con}}\left(M, V^{*}\right)$ and the second intersection is taken over all characters $\eta: \mathbb{Z}_{p}^{\times} \rightarrow L^{\times}$with $\eta^{2}=1$ and all $\xi \in \operatorname{Hom}_{\mathcal{O} \llbracket \mathrm{GL}_{2}\left(\mathbb{Z}_{p}\right) \rrbracket}^{\mathrm{cont}}\left(M,(V \otimes \eta \circ \mathrm{det})^{*}\right)$. 
Proof. Let $Z$ be the center of $\mathrm{GL}_{2}\left(\mathbb{Z}_{p}\right)$. The determinant induces the isomorphism $\mathrm{GL}_{2}\left(\mathbb{Z}_{p}\right) / Z \mathrm{SL}_{2}\left(\mathbb{Z}_{p}\right) \cong \mathbb{Z}_{p}^{\times} /\left(\mathbb{Z}_{p}^{\times}\right)^{2}$, which is a cyclic group of order 2 if $p \neq 2$, and a product of cyclic groups of order 2 if $p=2$. Hence, $\operatorname{Ind}_{Z \mathrm{ZS}_{2}\left(\mathbb{Z}_{p}\right)}^{\mathrm{GL}_{2}\left(\mathbb{Z}_{p}\right)} \mathbf{1} \cong \eta \circ$ det, where the sum is taken over all characters $\eta$ with $\eta^{2}=1$. The isomorphism

$$
\begin{aligned}
\operatorname{Hom}_{\mathcal{O} \llbracket \mathrm{SL}_{2}\left(\mathbb{Z}_{p}\right) \rrbracket}^{\text {cont }}\left(M, V^{*}\right) & \cong \operatorname{Hom}_{\mathcal{O} \llbracket Z \operatorname{SL}_{2}\left(\mathbb{Z}_{p}\right) \rrbracket}^{\text {cont }}\left(M, V^{*}\right) \\
& \cong \operatorname{Hom}_{\mathcal{O} \llbracket G_{2}\left(\mathbb{Z}_{p}\right) \rrbracket}^{\text {cont }}\left(M, V^{*} \otimes \operatorname{Ind}_{Z \mathrm{SL}_{2}\left(\mathbb{Z}_{p}\right)} \mathbf{1}\right) \\
& \cong \bigoplus_{\eta} \operatorname{Hom}_{\mathcal{O} \llbracket \mathrm{GL}_{2}\left(\mathbb{Z}_{p}\right) \rrbracket}^{\text {cont }}\left(M, V^{*} \otimes \eta \circ \text { det }\right)
\end{aligned}
$$

implies the assertion.

Lemma 2.6. Let $M \in \operatorname{Mod}_{\mathrm{GL}_{2}\left(\mathbb{Z}_{p}\right), \psi}^{\mathrm{pro}}(\mathcal{O})$ and let $\left\{V_{i}\right\}_{i \in I}$ be a family of continuous representations of $K$ on finite-dimensional $L$-vector spaces with a central character $\psi$. If $\left\{\left.V_{i}\right|_{\mathrm{SL}_{2}\left(\mathbb{Z}_{p}\right)}\right\}_{i \in I}$ captures $\left.M\right|_{\mathrm{SL}_{2}\left(\mathbb{Z}_{p}\right)}$ then $\left\{V_{i} \otimes \eta \circ \operatorname{det}\right\}_{i \in I, \eta}$ captures $M$, where $\eta$ runs over all characters $\eta: \mathbb{Z}_{p}^{\times} \rightarrow L^{\times}$with $\eta^{2}=1$.

Proof. The assertion follows from Lemma 2.5 and [Colmez et al. 2014, Lemma 2.7].

Proposition 2.7. Let $K=\mathrm{GL}_{2}\left(\mathbb{Z}_{p}\right)$, and let $Z$ be the center of $K$ and $\psi: Z \rightarrow L^{\times}$ a continuous character. There is a smooth irreducible representation $\tau$ of $K$ which is a type for a Bernstein component containing a principal series representation, but not containing a special series representation, such that

$$
\left\{\tau \otimes \operatorname{Sym}^{a} L^{2} \otimes \eta^{\prime} \circ \operatorname{det}\right\}_{a \in \mathbb{N}, \eta^{\prime}}
$$

captures every projective object in $\operatorname{Mod}_{K, \psi}^{\text {pro }}(\mathcal{O})$. Here, for each $a \in \mathbb{N}, \eta^{\prime}$ runs over all continuous characters $\eta^{\prime}: \mathbb{Z}_{p}^{\times} \rightarrow L^{\times}$such that $\tau \otimes \operatorname{Sym}^{a} L^{2} \otimes \eta^{\prime} \circ \operatorname{det}$ has central character $\psi$.

Proof. If $p \neq 2$ (resp. $p=2$ ) then $1+p \mathbb{Z}_{p}$ (resp. $1+4 \mathbb{Z}_{2}$ ) is a free pro- $p$ group of rank 1. Using this one may show that there is a smooth, nontrivial character $\chi: \mathbb{Z}_{p}^{\times} \rightarrow L^{\times}$and a continuous character $\eta_{0}: \mathbb{Z}_{p}^{\times} \rightarrow L^{\times}$such that $\psi=\chi \eta_{0}^{2}$. Let $e$ be the smallest integer such that $\chi$ is trivial on $1+p^{e} \mathbb{Z}_{p}$. Let

$$
J=\left(\begin{array}{cc}
\mathbb{Z}_{p}^{\times} & \mathbb{Z}_{p} \\
p^{e} \mathbb{Z}_{p} & \mathbb{Z}_{p}^{\times}
\end{array}\right)
$$

and let $\chi \otimes 1: J \rightarrow L^{\times}$be the character which sends $\left(\begin{array}{ll}a & b \\ c & d\end{array}\right) \mapsto \chi(a)$. The representation $\tau:=\operatorname{Ind}_{J}^{K}(\chi \otimes \mathbf{1})$ is irreducible and is a type. More precisely, for an irreducible smooth $\bar{L}$-representation $\pi$ of $G=\mathrm{GL}_{2}\left(\mathbb{Q}_{p}\right)$, we have $\operatorname{Hom}_{K}(\tau, \pi) \neq 0$ if and only if $\pi \cong\left(\operatorname{Ind}_{B}^{G} \psi_{1} \otimes \psi_{2}\right)_{\mathrm{sm}}$, where $B$ is a Borel subgroup and $\left.\psi_{1}\right|_{\mathbb{Z}_{p}^{\times}}=\chi$ and $\left.\psi_{2}\right|_{\mathbb{Z}_{p}^{\times}}=\mathbf{1}$; see [Henniart 2002, §A.2.2]. The central character of $\tau$ is equal to $\chi$. We claim that the family $\left\{\tau \otimes \operatorname{Sym}^{2 a} L^{2} \otimes(\operatorname{det})^{-a} \otimes \eta \eta_{0} \circ \operatorname{det}\right\}_{a \in \mathbb{N}, \eta}$, where $\eta$ runs 
over all the characters with $\eta^{2}=1$, captures every projective object in $\operatorname{Mod}_{K, \psi}^{\text {pro }}(\mathcal{O})$. If $M \in \operatorname{Mod}_{K, \psi}^{\text {pro }}(\mathcal{O})$ is projective then $\left.M\right|_{\mathrm{SL}_{2}\left(\mathbb{Z}_{p}\right)}$ is projective in $\operatorname{Mod}_{\mathrm{SL}_{2}\left(\mathbb{Z}_{p}\right), \psi}^{\text {pro }}(\mathcal{O})$ [Emerton 2010b, Proposition 2.1.11]. Lemma 2.4 implies that the family captures $\left.M\right|_{\mathrm{SL}_{2}\left(\mathbb{Z}_{p}\right)}$. Since each representation in the family has central character equal to $\chi \eta_{0}^{2}=\psi$, the claim follows from Lemma 2.6. Since the family of representations appearing in the claim is a subfamily of the representations appearing in the proposition, the claim implies the proposition.

2B. The image of Colmez's Montreal functor. Let $G=\mathrm{GL}_{2}\left(\mathbb{Q}_{p}\right), K=\mathrm{GL}_{2}\left(\mathbb{Z}_{p}\right)$. Let $B$ be the subgroup of upper-triangular matrices in $G$, let $T$ be the subgroup of diagonal matrices and let $Z$ be the center of $G$. We make no assumption on the prime $p$. We fix a continuous character $\psi: Z \rightarrow \mathcal{O}^{\times}$.

Let $\operatorname{Mod}_{G}^{\text {pro }}(\mathcal{O})$ be the category of profinite augmented representations of $G$ [Emerton 2010a, Definition 2.1.6]. The Pontryagin duality

$$
\pi \mapsto \pi^{\vee}:=\operatorname{Hom}_{\mathcal{O}}^{\text {cont }}(\pi, L / \mathcal{O})
$$

induces an antiequivalence of categories between $\operatorname{Mod}_{G}^{\mathrm{sm}}(\mathcal{O})$ and $\operatorname{Mod}_{G}^{\mathrm{pro}}(\mathcal{O})[$ Emerton 2010a, (2.2.8)]. Let $\operatorname{Mod}_{G}^{1 . \mathrm{adm}}(\mathcal{O})$ be the full subcategory of $\operatorname{Mod}_{G}^{\mathrm{sm}}(\mathcal{O})$ consisting of locally admissible [Emerton 2010a, Definition 2.2.17] representations of $G$ and let $\operatorname{Mod}_{G, \psi}^{1 . a d m}(\mathcal{O})$ be the full subcategory of $\operatorname{Mod}_{G}^{1 . \operatorname{adm}}(\mathcal{O})$ consisting of those representations on which $Z$ acts by the character $\psi$. Let $\mathfrak{C}(\mathcal{O})$ be the full subcategory of $\operatorname{Mod}_{G}^{\text {pro }}(\mathcal{O})$ antiequivalent to $\operatorname{Mod}_{G, \psi}^{1 . \operatorname{adm}}(\mathcal{O})$ via the Pontryagin duality. For $\pi_{1}, \pi_{2} \in \operatorname{Mod}_{G, \psi}^{\mathrm{l} . a d m}(\mathcal{O})$ we let $\operatorname{Ext}_{G, \psi}^{i}\left(\pi_{1}, \pi_{2}\right)$ be the Yoneda Ext group computed in $\operatorname{Mod}_{G, \psi}^{1 . \operatorname{adm}}(\mathcal{O})$.

Let $\pi \in \operatorname{Mod}_{G, \psi}^{1 . a d m}(\mathcal{O})$ be absolutely irreducible and either supersingular [Barthel and Livné 1994; Breuil 2003a] or a principal series representation isomorphic to $\left(\operatorname{Ind}_{B}^{G} \chi_{1} \otimes \chi_{2} \omega^{-1}\right)_{\text {sm }}$, for some smooth characters $\chi_{1}, \chi_{2}: \mathbb{Q}_{p}^{\times} \rightarrow k^{\times}$such that $\chi_{1} \chi_{2}^{-1} \neq \omega^{ \pm 1}, 1$. This hypothesis ensures that $\pi^{\prime}:=\left(\operatorname{Ind}_{B}^{G} \chi_{2} \otimes \chi_{1} \omega^{-1}\right)_{\text {sm }}$ is also absolutely irreducible and $\pi ¥ \pi^{\prime}$. Let $P \rightarrow \pi^{\vee}$ be a projective envelope of $\pi^{\vee}$ in $\mathfrak{C}(\mathcal{O})$ and let $E=\operatorname{End}_{\mathfrak{C}(\mathcal{O})}(P)$. Then $E$ is naturally a topological ring with a unique maximal ideal and residue field $k=\operatorname{End}_{\mathfrak{C}(\mathcal{O})}\left(\pi^{\vee}\right)$; see [Paškūnas 2013, §2].

Proposition 2.8. If $\pi$ is supersingular then $k \widehat{\otimes}_{E} P \cong \pi^{\vee}$. If $\pi$ is a principal series then $k \widehat{\otimes}_{E} P \cong \kappa^{\vee}$, where $\kappa$ is the unique nonsplit extension $0 \rightarrow \pi \rightarrow \kappa \rightarrow \pi^{\prime} \rightarrow 0$. Proof. In both cases, $\left(k \widehat{\otimes}_{E} P\right)^{\vee}$ is the unique representation in $\operatorname{Mod}_{G, \psi}^{1 . a d m}(\mathcal{O})$ which is maximal with respect to the following conditions: (1) $\operatorname{soc}_{G}\left(k \widehat{\otimes}_{E} P\right)^{\vee} \cong \pi$; (2) $\pi$ occurs in $\left(k \widehat{\otimes}_{E} P\right)^{\vee}$ with multiplicity one; see [Paškūnas 2013, Remark 1.13]. For, if $\tau \in \operatorname{Mod}_{G, \psi}^{1 . a d m}(\mathcal{O})$ satisfies both conditions, then (1) and [Paškūnas 2013, Lemma 2.10] imply that the natural map $\operatorname{Hom}_{\mathfrak{C}(\mathcal{O})}\left(P, \tau^{\vee}\right) \widehat{\otimes}_{E} P \rightarrow \tau^{\vee}$ is surjective, and (2) and the exactness of $\operatorname{Hom}_{\mathfrak{C}(\mathcal{O})}(P, *)$ imply that $\operatorname{Hom}_{\mathfrak{C}(\mathcal{O})}\left(P, \tau^{\vee}\right) \cong$ $\operatorname{Hom}_{\mathfrak{C}(\mathcal{O})}\left(P, \pi^{\vee}\right) \cong k$. Hence, dually we obtain an injection $\tau \hookrightarrow\left(k \widehat{\otimes}_{E} P\right)^{\vee}$. 
Let $\pi_{1}$ be an irreducible representation in $\operatorname{Mod}_{G, \psi}^{1 . a d m}(\mathcal{O})$ such that $\operatorname{Ext}_{G, \psi}^{1}\left(\pi_{1}, \pi\right)$ is nonzero. It follows from Corollary 1.2 in [Paškūnas 2014] that if $\pi$ is supersingular then $\pi_{1} \cong \pi$ and hence $\left(k \widehat{\otimes}_{E} P\right)^{\vee} \cong \pi$, and if $\pi$ is a principal series as above then $\pi_{1} \cong \pi$ or $\pi_{1} \cong \pi^{\prime}$. We will now explain how to modify the arguments of [Paškūnas 2013, §8] so that they also work for $p=2$, the main point being that Emerton's functor of ordinary parts works for all $p$. Proposition 4.3.15(2) of [Emerton 2010b] implies that $\operatorname{Ext}_{G, \psi}^{1}\left(\pi^{\prime}, \pi\right)$ is one-dimensional. Let $\kappa$ be the unique nonsplit extension $0 \rightarrow \pi \rightarrow \kappa \rightarrow \pi^{\prime} \rightarrow 0$. We claim that $\operatorname{Ext}_{G, \psi}^{n}\left(\pi^{\prime}, \kappa\right)=0$ for all $n \geq 0$. The claim for $n=1$ implies that $\left(k \widehat{\otimes}_{E} P\right)^{\vee} \cong \kappa$. It is proved in [Emerton and Paškūnas 2010, Corollary 3.12] that the $\delta$-functor $H^{\bullet} \operatorname{Ord}_{B}$, defined in [Emerton 2010b, Definition 3.3.1], is effaceable in $\operatorname{Mod}_{G, \psi}^{\text {l.adm }}(\mathcal{O})$. Hence it coincides with the derived functor $\mathbb{R}^{\bullet} \operatorname{Ord}_{B}$. An open compact subgroup $N_{0}$ of the unipotent radical of $B$ is isomorphic to $\mathbb{Z}_{p}$, and hence $H^{i}\left(N_{0}, *\right)$ vanishes for $i \geq 2$. This implies that $\mathbb{R}^{i} \operatorname{Ord}_{B}=H^{i} \operatorname{Ord}_{B}=0$ for $i \geq 2$. The proof of [Paškūnas 2013, Lemma 8.1] does not use the assumption $p>2$ and gives that

$$
\operatorname{Ord}_{B} \kappa \cong \operatorname{Ord}_{B} \pi \cong \mathbb{R}^{1} \operatorname{Ord}_{B} \pi^{\prime} \cong \mathbb{R}^{1} \operatorname{Ord}_{B} \kappa \cong \chi_{2} \omega^{-1} \otimes \chi_{1} .
$$

Our assumption on $\chi_{1}$ and $\chi_{2}$ implies that $\chi_{1} \omega^{-1} \otimes \chi_{2}$ and $\chi_{2} \omega^{-1} \otimes \chi_{1}$ are distinct characters of $T$. It follows from [Emerton 2010b, Lemma 4.3.10] that all the Ext-groups between them vanish. Since $\pi^{\prime} \cong\left(\operatorname{Ind}_{\bar{B}}^{G} \chi_{1} \omega^{-1} \otimes \chi_{2}\right)_{\text {sm }}$, where $\bar{B}$ is the subgroup of lower-triangular matrices in $G$, all the terms in Emerton's spectral sequence [2010b, (3.7.4)] converging to $\operatorname{Ext}_{G, \psi}^{n}\left(\pi^{\prime}, \kappa\right)$ are zero. Hence, $\operatorname{Ext}_{G, \psi}^{n}\left(\pi_{2}, \kappa\right)=0$ for all $n \geq 0$. Let us also note that the 5 -term exact sequence associated to the spectral sequence implies that $\operatorname{Ext}_{G, \psi}^{1}(\pi, \kappa)$ is finite-dimensional.

Proposition 2.9. If $\pi$ is supersingular then let $S=Q=\pi^{\vee}$. If $\pi$ is a principal series then let $S=\pi^{\vee}$ and $Q=\kappa^{\vee}$. Then $S$ and $Q$ satisfy the hypotheses (H0)-(H5) of [Paškūnas 2013, §3].

Proof. If $\pi$ is supersingular then there are no other irreducible representations in the block of $\pi$ and hence the only hypothesis to check is (H4), which is equivalent to the finite-dimensionality of $\operatorname{Ext}_{G, \psi}^{1}(\pi, \pi)$. This follows from Proposition 9.1 in [Paškūnas 2010b]. If $\pi$ is a principal series then the assertion follows from the Ext-group calculations made in the proof of Proposition 2.8.

The proposition enables us to apply the formalism developed in [Paškūnas 2013, Section 3]. Corollary 3.12 of [Paškūnas 2013] implies:

Proposition 2.10. The functor $\widehat{\otimes}_{E} P$ is an exact functor from the category of pseudocompact right E-modules to $\mathfrak{C}(\mathcal{O})$. 
If $\mathrm{m}$ is a pseudocompact right $E$-module then $\operatorname{Hom}_{\mathfrak{C}(\mathcal{O})}\left(P, \mathrm{~m} \widehat{\otimes}_{E} P\right) \cong \mathrm{m}$ by [Paškūnas 2013, Lemma 2.9]. This implies that the functor is fully faithful, so that

$$
\operatorname{Hom}_{E}^{\text {cont }}\left(\mathrm{m}_{1}, \mathrm{~m}_{2}\right) \cong \operatorname{Hom}_{\mathfrak{C}(\mathcal{O})}\left(\mathrm{m}_{1} \widehat{\otimes}_{E} P, \mathrm{~m}_{2} \widehat{\otimes}_{E} P\right) .
$$

\section{Proposition 2.11. E is commutative.}

Proof. Let $\widetilde{\mathfrak{C}}(\mathcal{O})$ be the full subcategory of $\operatorname{Mod}_{G}^{\text {pro }}(\mathcal{O})$ which is antiequivalent to $\operatorname{Mod}_{G}^{1 . a d m}(\mathcal{O})$ via the Pontryagin duality. Let $\widetilde{P}$ be a projective envelope of $\pi^{\vee}$ in $\widetilde{\mathfrak{C}}(\mathcal{O})$, let $\widetilde{E}:=\operatorname{End}_{\widetilde{\mathfrak{C}}(\mathcal{O})}(\widetilde{P})$ and let $\mathfrak{a}$ be the closed two-sided ideal of $\widetilde{E}$ generated by the elements $z-\psi^{-1}(z)$, for all $z$ in the center of $G$. We may consider $\mathfrak{C}(\mathcal{O})$ as a full subcategory of $\widetilde{\mathfrak{C}}(\mathcal{O})$. Since the center of $G$ acts on $\widetilde{P} / \mathfrak{a} \widetilde{P}$ by $\psi^{-1}$, we have $\widetilde{P} / \mathfrak{a} \widetilde{P} \in \mathfrak{C}(\mathcal{O})$. The functor $\operatorname{Hom}_{\mathfrak{C}(\mathcal{O})}(\widetilde{P} / \mathfrak{a} \widetilde{P}, *)$ is exact, since

$$
\operatorname{Hom}_{\mathfrak{C}(\mathcal{O})}(\widetilde{P} / \mathfrak{a} \widetilde{P}, M)=\operatorname{Hom}_{\widetilde{\mathfrak{C}}(\mathcal{O})}(\widetilde{P}, M)
$$

for all $M \in \mathfrak{C}(\mathcal{O})$, and $\widetilde{P}$ is projective. Hence, $\widetilde{P} / \mathfrak{a} \widetilde{P}$ is projective in $\mathfrak{C}(\mathcal{O})$. Its $G$-cosocle is isomorphic to $\pi^{\vee}$, since the same is true of $\widetilde{P}$. Hence, $\widetilde{P} / \mathfrak{a} \widetilde{P}$ is a projective envelope of $\pi^{\vee}$ in $\mathfrak{C}(\mathcal{O})$. Since projective envelopes are unique up to isomorphism, $\widetilde{P} / \mathfrak{a} \widetilde{P}$ is isomorphic to $P$. Since $\mathfrak{a}$ is generated by central elements, any $\phi \in \widetilde{E}$ maps $\mathfrak{a} \widetilde{P}$ to itself. This yields a ring homomorphism $\widetilde{E} \rightarrow \operatorname{End}_{\mathfrak{C}(\mathcal{O})}(\widetilde{P} / \mathfrak{a} \widetilde{P}) \cong E$. Projectivity of $\widetilde{P}$ and (4) applied with $M=\widetilde{P} / \mathfrak{a} \widetilde{P}$ implies that the homomorphism is surjective and induces an isomorphism $\widetilde{E} / \mathfrak{a} \cong \operatorname{End}_{\mathfrak{C}(\mathcal{O})}(\widetilde{P} / \mathfrak{a} \widetilde{P})$. Since $\widetilde{E}$ is commutative [Colmez et al. 2014, Corollary 2.22] we deduce that $E$ is commutative.

Proposition 2.12. E is a complete local noetherian commutative $\mathcal{O}$-algebra with residue field $k$.

Proof. Proposition 2.11 asserts that $E$ is commutative. Corollary 3.11 of [Paškūnas 2013] implies that the natural topology on $E$ (see [Paškūnas 2013, §2]) coincides with the topology defined by the maximal ideal $\mathfrak{m}$, which implies that $E$ is complete for the $m$-adic topology. It follows from Lemma 3.7, Proposition 3.8(iii) of [Paškūnas 2013] that $\mathfrak{m} /\left(\mathfrak{m}^{2}+(\varpi)\right)$ is a finite-dimensional $k$-vector space. Since $E$ is commutative, we deduce that $E$ is noetherian.

Proposition 2.13. Let $Q=\pi^{\vee}$ if $\pi$ is supersingular and let $Q=\kappa^{\vee}$ if $\pi$ is a principal series. The ring $E$ represents the universal deformation problem of $Q$ in $\mathfrak{C}(\mathcal{O})$, and $P$ is the universal deformation of $Q$.

Proof. Since $E$ is commutative by Proposition 2.11 and since hypotheses (H0)-(H5) of [Paškūnas 2013, §3] are satisfied by Proposition 2.9, the assertion follows from [Paškūnas 2013, Corollary 3.27]. 
2B1. Colmez's Montreal functor. This subsection is essentially the same as Section 5.7 of [Paškūnas 2013]. Let $G_{\mathbb{Q}_{p}}$ be the absolute Galois group of $\mathbb{Q}_{p}$. We will consider $\psi$ as a character of $G_{\mathbb{Q}_{p}}$ via local class field theory, normalized so that the uniformizers correspond to geometric Frobenii. Let $\varepsilon: G_{\mathbb{Q}_{p}} \rightarrow \mathcal{O}^{\times}$be the $p$-adic cyclotomic character. Similarly, we will identify $\varepsilon$ with the character of $\mathbb{Q}_{p}^{\times}$, which maps $x$ to $x|x|$.

Colmez [2010] has defined an exact and covariant functor $\boldsymbol{V}$ from the category of smooth, finite-length representations of $G$ on $\mathcal{O}$-torsion modules with a central character to the category of continuous finite-length representations of $G_{\mathbb{Q}_{p}}$ on $\mathcal{O}$-torsion modules. This functor enables us to make the connection between the $\mathrm{GL}_{2}\left(\mathbb{Q}_{p}\right)$ and $G_{\mathbb{Q}_{p}}$ worlds. We modify Colmez's functor to obtain an exact covariant functor

$$
\check{\boldsymbol{V}}: \mathfrak{C}(\mathcal{O}) \rightarrow \operatorname{Mod}_{G_{\mathbb{Q}_{p}}}^{\text {pro }}(\mathcal{O})
$$

as follows. Let $M$ be in $\mathfrak{C}(\mathcal{O})$. If it is of finite length then $\check{V}(M):=\boldsymbol{V}\left(M^{\vee}\right)^{\vee}(\varepsilon \psi)$, where $\vee$ denotes the Pontryagin dual and $\varepsilon$ is the cyclotomic character. In general, we may write $M \cong \lim M_{i}$, where the limit is taken over all quotients of finite length in $\mathfrak{C}(\mathcal{O})$, and we define $\check{V}(M):=\lim _{\longleftarrow} \check{V}\left(M_{i}\right)$. If $\pi \in \operatorname{Mod}_{G, \psi}^{1 . f i n}(k)$ is absolutely irreducible, then $\pi^{\vee}$ is an object of $\mathfrak{C}(\mathcal{O})$, and if $\pi$ is supersingular in the sense of [Barthel and Livné 1994], then $\check{V}\left(\pi^{\vee}\right) \cong V(\pi)$ is an absolutely irreducible continuous representation of $G_{\mathbb{Q}_{p}}$ associated to $\pi$ by Breuil [2003a]. If $\pi \cong$ $\left(\operatorname{Ind}_{B}^{G} \chi_{1} \otimes \chi_{2} \omega^{-1}\right)_{\text {sm }}$ then $\check{V}\left(\pi^{\vee}\right) \cong \chi_{1}$. If $\pi \cong \chi \circ$ det then $\check{V}\left(\pi^{\vee}\right)=0$ and if $\pi \cong \mathrm{Sp} \otimes \chi \circ$ det, where Sp is the Steinberg representation, then $\check{V}\left(\pi^{\vee}\right) \cong \chi$. Since $\check{V}$ is exact we obtain an exact sequence of $G_{\mathbb{Q}_{p}}$-representations

$$
0 \rightarrow \chi_{2} \rightarrow \check{V}\left(\kappa^{\vee}\right) \rightarrow \chi_{1} \rightarrow 0 .
$$

The sequence is nonsplit by [Colmez 2010, Proposition VII.4.13(iii)]. If $\mathrm{m}$ is a pseudocompact right $E$-module then there exists a natural isomorphism of $G_{\mathbb{Q}_{p}}{ }^{-}$ representations

$$
\check{\boldsymbol{V}}\left(\mathrm{m} \widehat{\otimes}_{E} P\right) \cong \mathrm{m} \widehat{\otimes}_{E} \check{\boldsymbol{V}}(P),
$$

by [Paškūnas 2013, Lemma 5.53]. It follows from (6) and Proposition 2.10 that $\check{\boldsymbol{V}}(P)$ is a deformation of $\rho:=\check{\boldsymbol{V}}\left(k \widehat{\otimes}_{E} P\right)$ to $E$. If $\pi$ is supersingular then $\rho$ is an absolutely irreducible 2-dimensional representation of $G_{\mathbb{Q}_{p}}$, and if $\pi$ is a principal series then $\rho$ is a nonsplit extension of distinct characters; see (5). In both cases, $\operatorname{End}_{G_{\mathbb{Q}_{p}}}(\rho)=k$ and so the universal deformation problem of $\rho$ is represented by a complete local noetherian $\mathcal{O}$-algebra $R$. Let $R^{\psi}$ be the quotient of $R$ parametrizing deformations of $\rho$ with determinant equal to $\psi \varepsilon$.

Proposition 2.14. The functor $\check{V}$ induces surjective homomorphisms $R \rightarrow E$ and $\varphi: E \rightarrow R^{\psi}$. 
Proof. This is proved in the same way as [Paškūnas 2013, Proposition 5.56, §5.8], following [Kisin 2010]. For the first surjection it is enough to prove that $\check{\boldsymbol{V}}$ induces an injection

$$
\operatorname{Ext}_{\mathfrak{C}(\mathcal{O})}^{1}(Q, Q) \hookrightarrow \operatorname{Ext}_{G_{\mathbb{Q}_{p}}}^{1}(\rho, \rho) .
$$

This follows from [Colmez 2010, Théorème VII.5.2]. To prove the second surjection, we observe that $R^{\psi}$ is reduced and $\mathcal{O}$-torsion-free: if $p \geq 5$ then $R^{\psi}$ is formally smooth over $\mathcal{O}$, if $p=3$ then the assertion follows from results of [Böckle 2010], and if $p=2$ then the assertion follows from [Chenevier 2009, Proposition 4.1]. Thus it is enough to show that every closed point of Spec $R^{\psi}[1 / p]$ is contained in Spec $E$. This is equivalent to showing that for every deformation $\tilde{\rho}$ of $\rho$ with determinant $\psi \varepsilon$ there is a Banach space representation $\Pi$ lifting $Q^{\vee}$ with central character $\psi$ such that $\check{V}(\Pi) \cong \tilde{\rho}$. This follows from [Colmez et al. 2015, Theorem 10.1].

2B2. Banach space representations. Let $\operatorname{Ban}_{G, \psi}^{\mathrm{adm}}(L)$ be the category of admissible unitary $L$-Banach space representations [Schneider and Teitelbaum 2002, §3] on which $Z$ acts by the character $\psi$. If $\Pi \in \operatorname{Ban}_{G, \psi}^{\operatorname{adm}}(L)$ then we let

$$
\check{\boldsymbol{V}}(\Pi):=\check{\boldsymbol{V}}\left(\Theta^{d}\right) \otimes_{\mathcal{O}} L,
$$

where $\Theta$ is any open bounded $G$-invariant lattice in $\Pi$. Therefore, $\check{V}$ is exact and contravariant on $\operatorname{Ban}_{G, \psi}^{\mathrm{adm}}(L)$.

Remark 2.15. One of the reasons we use $\check{V}$ instead of $\boldsymbol{V}$ is that this allows us to define $\check{V}(\Pi)$ without making the assumption that the reduction of $\Pi$ modulo $\varpi$ has finite length as a $G$-representation.

If $\mathrm{m}$ is an $E[1 / p]$-module of finite length then we let

$$
\Pi(\mathrm{m}):=\operatorname{Hom}_{\mathcal{O}}^{\text {cont }}\left(\mathrm{m}^{0} \widehat{\otimes}_{E} P, L\right),
$$

where $\mathrm{m}^{0}$ is any $E$-stable $\mathcal{O}$-lattice in $\mathrm{m}$. Then $\Pi(\mathrm{m})$ is an admissible unitary $L$-Banach space representation of $G$, by [Paškūnas 2015b, Lemma 2.21], with the topology given by the supremum norm. Since the functor $\widehat{\otimes}_{E} P$ is exact by Proposition 2.10, the functor $\mathrm{m} \mapsto \Pi(\mathrm{m})$ is exact and contravariant. Moreover, it is fully faithful, as

$$
\begin{aligned}
\operatorname{Hom}_{G}\left(\Pi\left(\mathrm{m}_{1}\right), \Pi\left(\mathrm{m}_{2}\right)\right) & \cong \operatorname{Hom}_{\mathfrak{C}(\mathcal{O})}\left(\mathrm{m}_{2}^{0} \widehat{\otimes}_{E} P, \mathrm{~m}_{1}^{0} \widehat{\otimes}_{E} P\right)_{L} \\
& \cong \operatorname{Hom}_{E[1 / p]}\left(\mathrm{m}_{2}, \mathrm{~m}_{1}\right),
\end{aligned}
$$

where the first isomorphism follows from Theorem 2.3 of [Schneider and Teitelbaum 2002] and the second from (3).

Lemma 2.16. Let $\mathrm{m}$ be an $E[1 / p]$-module of finite length and let $\Pi \in \operatorname{Ban}_{G, \psi}^{\mathrm{adm}}(L)$ be such that $\pi$ does not occur as a subquotient in the reduction of an open bounded 


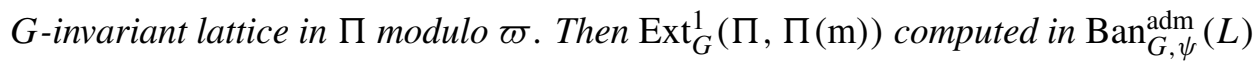
is zero.

Proof. If $\Theta$ is an open bounded $G$-invariant lattice in $\mathrm{B} \in \operatorname{Ban}_{G, \psi}^{\mathrm{adm}}(L)$ then we define $\mathrm{m}(\mathrm{B}):=\operatorname{Hom}_{\mathfrak{C}(\mathcal{O})}\left(P, \Theta^{d}\right)_{L}$. Proposition 4.17 in [Paškūnas 2013] implies that $\mathrm{m}(\mathrm{B})$ is a finitely generated $E[1 / p]$-module. The functor $\mathrm{B} \mapsto \mathrm{m}(\mathrm{B})$ is exact by [Paškūnas 2013, Lemma 4.9]. The evaluation map $\operatorname{Hom}_{\mathfrak{C}(\mathcal{O})}\left(P, \Theta^{d}\right) \widehat{\otimes}_{E} P \rightarrow \Theta^{d}$ induces a continuous $G$-equivariant map $\mathrm{B} \rightarrow \Pi(\mathrm{m}(\mathrm{B}))$. If $\mathrm{m}$ is an $E[1 / p]$-module of finite length and $\mathrm{B} \cong \Pi(\mathrm{m})$ then $\mathrm{m}(\mathrm{B}) \cong \mathrm{m}$ and the map $\mathrm{B} \rightarrow \Pi(\mathrm{m}(\mathrm{B}))$ is an isomorphism by [Paškūnas 2013, Lemma 4.28]. Moreover, $\mathrm{m}(\mathrm{B})=0$ if and only if $\pi$ does not occur as a subquotient of $\Theta /(\varpi)$, by [Colmez et al. 2014, Proposition 2.1(ii)]. Hence, if we have an exact sequence $0 \rightarrow \Pi(\mathrm{m}) \rightarrow \mathrm{B} \rightarrow \Pi \rightarrow 0$ then by applying the functor $\mathrm{m}$ to it, we obtain an isomorphism $\mathrm{m} \cong \mathrm{m}(\Pi(\mathrm{m})) \cong \mathrm{m}(B)$ and hence an isomorphism $\Pi(\mathrm{m}) \cong \Pi(\mathrm{m}(\mathrm{B}))$. The map $\mathrm{B} \rightarrow \Pi(\mathrm{m}(\mathrm{B}))$ splits the exact sequence.

The proof of [Paškūnas 2015b, Lemma 4.3] shows that we have a natural isomorphism of $G_{\mathbb{Q}_{p}}$-representations

$$
\check{\boldsymbol{V}}(\Pi(\mathrm{m})) \cong \mathrm{m} \otimes_{E} \check{\boldsymbol{V}}(P) .
$$

Let us point out a special case of this isomorphism. If $\mathfrak{n}$ is a maximal ideal of $E[1 / p]$ then its residue field $\kappa(\mathfrak{n})$ is a finite extension of $L$. Let $\mathcal{O}_{\kappa(\mathfrak{n})}$ be the ring of integers in $\kappa(\mathfrak{n})$ and let $\varpi_{\kappa(\mathfrak{n})}$ be the uniformizer. Then $\Theta:=\operatorname{Hom}_{\mathcal{O}}^{\text {cont }}\left(\mathcal{O}_{\kappa(\mathfrak{n})} \widehat{\otimes}_{E} P, \mathcal{O}\right)$ is an open bounded $G$-invariant lattice in $\Pi(\kappa(\mathfrak{n}))$. The evaluation map induces an isomorphism $\Theta^{d} \cong \mathcal{O}_{\kappa(\mathfrak{n})} \widehat{\otimes}_{E} P$. Since $E$ is noetherian, $\mathcal{O}_{\kappa(\mathfrak{n})}$ is a finitely presented $E$-module and thus the usual and completed tensor products coincide. We obtain

$$
\check{\boldsymbol{V}}\left(\Theta^{d}\right) \cong \mathcal{O}_{\kappa(\mathfrak{n})} \otimes_{E} \check{\boldsymbol{V}}(P), \quad \check{\boldsymbol{V}}(\Pi(\kappa(\mathfrak{n}))) \cong \kappa(\mathfrak{n}) \otimes_{E} \check{\boldsymbol{V}}(P) .
$$

Since the residue field of $\mathcal{O}_{\kappa(\mathfrak{n})}$ is $k$, we have

$$
\Theta /\left(\varpi_{\kappa(\mathfrak{n})}\right) \cong \operatorname{Hom}_{k}^{\text {cont }}\left(k \widehat{\otimes}_{E} P, k\right) \cong\left(k \widehat{\otimes}_{E} P\right)^{\vee} .
$$

Recall from [Paškūnas 2013, §4] that $\Pi \in \operatorname{Ban}_{G, \psi}^{\mathrm{adm}}(L)$ is irreducible if it does not have a nontrivial closed $G$-invariant subspace. It is absolutely irreducible if $\Pi \otimes_{L} L^{\prime}$ is irreducible in $\operatorname{Ban}_{G, \psi}^{\mathrm{adm}}\left(L^{\prime}\right)$ for every finite field extension $L^{\prime} / L$. An irreducible $\Pi$ is ordinary if it is a subquotient of a unitary parabolic induction of a unitary character. Otherwise it is called nonordinary.

Proposition 2.17. If $\mathfrak{n}$ is a maximal ideal of $E[1 / p]$ then either the $\kappa(\mathfrak{n})$-Banach space representation $\Pi(\kappa(\mathfrak{n}))$ is absolutely irreducible nonordinary or

$$
\pi \cong\left(\operatorname{Ind}_{B}^{G} \chi_{1} \otimes \chi_{2} \omega^{-1}\right)_{\mathrm{sm}}
$$


and (after possibly replacing $\kappa(\mathfrak{n})$ by a finite extension) there exists a nonsplit extension

$$
0 \rightarrow\left(\operatorname{Ind}_{B}^{G} \delta_{1} \otimes \delta_{2} \varepsilon^{-1}\right)_{\text {cont }} \rightarrow \Pi(\kappa(\mathfrak{n})) \rightarrow\left(\operatorname{Ind}_{B}^{G} \delta_{2} \otimes \delta_{1} \varepsilon^{-1}\right)_{\text {cont }} \rightarrow 0,
$$

where $\delta_{1}, \delta_{2}: \mathbb{Q}_{p}^{\times} \rightarrow \kappa(\mathfrak{n})^{\times}$are unitary characters congruent to $\chi_{1}$ and $\chi_{2}$, respectively, such that $\delta_{1} \delta_{2}=\psi \varepsilon$.

Proof. It follows from (11) that $\operatorname{dim}_{\kappa(\mathfrak{n})} \check{V}(\Pi(\kappa(\mathfrak{n})))=2$. Since $\check{\boldsymbol{V}}$ applied to a parabolic induction of a unitary character is a one-dimensional representation of $G_{\mathbb{Q}_{p}}$, we deduce that if $\Pi(\kappa(\mathfrak{n}))$ is absolutely irreducible then it cannot be ordinary.

If $\pi$ is supersingular then (12) implies that $\Theta /\left(\varpi_{\kappa(\mathfrak{n})}\right) \cong \pi$, which is absolutely irreducible. This implies that $\Pi(\kappa(\mathfrak{n}))$ is absolutely irreducible. If $\pi$ is a principal series then $\Theta /\left(\varpi_{\kappa(\mathfrak{n})}\right)$ is of length 2 and both irreducible subquotients are absolutely irreducible. Hence, $\Pi(\kappa(\mathfrak{n}))$ is either irreducible or of length 2 . Let us assume that $\Pi(\kappa(\mathfrak{n}))$ is not absolutely irreducible. Then after possibly replacing $\kappa(\mathfrak{n})$ by a finite extension we have an exact sequence of admissible $\kappa(\mathfrak{n})$-Banach space representations $0 \rightarrow \Pi_{1} \rightarrow \Pi(\kappa(\mathfrak{n})) \rightarrow \Pi_{2} \rightarrow 0$. This sequence is nonsplit, since otherwise $\check{V}(\Pi(\kappa(\mathfrak{n})))$ would be a direct sum of two one-dimensional representations, which would contradict [Paškūnas 2015b, Lemma 4.5(iii)]. Let $\Theta_{1}:=\Theta \cap \Pi_{1}$ and let $\Theta_{2}$ be the image of $\Theta$ in $\Pi_{2}$. Since we are dealing with admissible representations, $\Theta_{2}$ is a bounded $\mathcal{O}$-lattice in $\Pi_{2}$. Lemma 5.5 of [Paškūnas 2010a] says that we have the exact sequences of $\mathcal{O}_{\kappa(\mathfrak{n})}$-modules

$$
\begin{gathered}
0 \rightarrow \Theta_{1} \rightarrow \Theta \rightarrow \Theta_{2} \rightarrow 0, \\
0 \rightarrow \Theta_{1} /\left(\varpi_{\kappa(\mathfrak{n})}\right) \rightarrow \Theta /\left(\varpi_{\kappa(\mathfrak{n})}\right) \rightarrow \Theta_{2} /\left(\varpi_{\kappa(\mathfrak{n})}\right) \rightarrow 0 .
\end{gathered}
$$

It follows from (12) that the exact sequence of $G$-representations in (15) is the unique nonsplit extension $0 \rightarrow \pi \rightarrow \kappa \rightarrow \pi^{\prime} \rightarrow 0$. Proposition 4.2.14 of [Emerton 2010b] applied with $A=\mathcal{O}_{\kappa(\mathfrak{n})} /\left(\varpi_{\kappa(\mathfrak{n})}^{n}\right)$ for all $n \geq 1$ implies that

$$
\Pi_{1} \cong\left(\operatorname{Ind}_{B}^{G} \delta_{1} \otimes \delta_{2} \varepsilon^{-1}\right)_{\text {cont }}, \quad \Pi_{2} \cong\left(\operatorname{Ind}_{B}^{G} \delta_{2}^{\prime} \otimes \delta_{1}^{\prime} \varepsilon^{-1}\right)_{\text {cont }},
$$

where $\delta_{1}, \delta_{2}, \delta_{1}^{\prime}, \delta_{2}^{\prime}: \mathbb{Q}_{p}^{\times} \rightarrow \kappa(\mathfrak{n})^{\times}$are unitary characters with $\delta_{1}, \delta_{1}^{\prime}$ congruent to $\chi_{1}$ and $\delta_{2}, \delta_{2}^{\prime}$ congruent to $\chi_{2}$ modulo $\varpi_{\kappa(\mathfrak{n})}$. We reduce (14) modulo $\varpi_{\kappa(\mathfrak{n})}^{n}$ to obtain an exact sequence to which we apply $\operatorname{Ord}_{B}$. This gives us an injection $\operatorname{Ord}_{B}\left(\Theta_{2} /\left(\varpi_{\kappa(\mathfrak{n})}^{n}\right)\right) \hookrightarrow \mathbb{R}^{1} \operatorname{Ord}_{B}\left(\Theta_{2} /\left(\varpi_{\kappa(\mathfrak{n})}^{n}\right)\right)$. Since both are free $\mathcal{O}_{\kappa(\mathfrak{n})} /\left(\varpi_{\kappa(\mathfrak{n})}^{n}\right)$ modules of rank 1 , the injection is an isomorphism. This implies that $\delta_{1}$ is congruent to $\delta_{1}^{\prime}$ and $\delta_{2}$ is congruent $\delta_{2}^{\prime}$ modulo $\varpi_{\kappa(\mathfrak{n})}^{n}$ for all $n \geq 1$. Hence, $\delta_{1}=\delta_{1}^{\prime}$ and $\delta_{2}=\delta_{2}^{\prime}$.

2B3. Main local result. We will prove that the surjection $\varphi: E \rightarrow R^{\psi}$ in Proposition 2.14 is an isomorphism. The argument combines the first part of the paper with methods of [Paškūnas 2015b]. The argument in [Paškūnas 2013] used to prove this statement when $p \geq 5$ uses the fact that the rings $R^{\psi}$ are formally smooth in that 
case. This does not hold in general; when $p=2$ or 3 and even when the ring is formally smooth and $p=3$, the computations just get too complicated.

Let $V$ be a continuous representation of $K$ with a central character $\psi$ of the form $\tau \otimes \operatorname{Sym}^{a} L^{2} \otimes \eta \circ$ det, where $\eta: \mathbb{Z}_{p}^{\times} \rightarrow L^{\times}$is a continuous character, and $\tau$ is a type for a Bernstein component containing a principal series representation, but not containing a special series representation.

Proposition 2.18. If $\mathfrak{n}$ is a maximal ideal of $E[1 / p]$ then the following hold:

(i) $\operatorname{dim}_{\kappa(\mathfrak{n})} \operatorname{Hom}_{K}(V, \Pi(\kappa(\mathfrak{n}))) \leq 1$.

(ii) $\operatorname{dim}_{\kappa(\mathfrak{n})} \operatorname{Hom}_{K}\left(V, \Pi\left(E_{\mathfrak{n}} / \mathfrak{n}^{2}\right)\right) \leq 2$.

Moreover, if $\operatorname{Hom}_{K}(V, \Pi(\kappa(\mathfrak{n}))) \neq 0$ then $\operatorname{det} \check{V}(\Pi(\kappa(\mathfrak{n})))=\psi \varepsilon$.

Proof. If $\mathrm{m}$ is an $E[1 / p]$-module of finite length and $L^{\prime}$ is a finite extension of $L$, then $\Pi\left(\mathrm{m} \otimes_{L} L^{\prime}\right) \cong \Pi(\mathrm{m}) \otimes_{L} L^{\prime}$ and $\operatorname{Hom}_{K}(V, \Pi(\mathrm{m})) \otimes_{L} L^{\prime} \cong \operatorname{Hom}_{K}\left(V, \Pi(\mathrm{m}) \otimes_{L} L^{\prime}\right)$. This implies that it is enough to prove the assertions after replacing $\kappa(\mathfrak{n})$ by a finite extension. In particular, we may assume that $\Pi(\kappa(\mathfrak{n}))$ is either absolutely irreducible or a nonsplit extension as in Proposition 2.17. Since $\check{V}$ is compatible with twisting by characters, to prove the proposition it is enough to assume that $\eta$ is trivial, so that $V$ is a locally algebraic representation of $K$.

Since $\tau$ is a type and $\Pi(\kappa(\mathfrak{n}))$ is admissible, $\operatorname{Hom}_{K}(V, \Pi(\kappa(\mathfrak{n}))) \neq 0$ if and only if (after possibly replacing $\kappa(\mathfrak{n})$ by a finite extension) $\Pi(\kappa(\mathfrak{n}))$ contains a subrepresentation of the form $\Psi \otimes \operatorname{Sym}^{a} L^{2}$, where $\Psi$ is an absolutely irreducible smooth principal series representation in the Bernstein component described by $\tau$; see the proof of [Paškūnas 2010a, Theorem 7.2]. Let $\Pi$ be the universal unitary completion of $\Psi \otimes \operatorname{Sym}^{a} L^{2}$. Then $\Pi$ is absolutely irreducible, by [Berger and Breuil 2010, Corollaire 5.3.4] and [Breuil and Emerton 2010, Proposition 2.2.1].

If $\Pi(\kappa(\mathfrak{n}))$ is absolutely irreducible, we deduce that $\Pi(\kappa(\mathfrak{n})) \cong \Pi$. Since $\Pi$ in [Berger and Breuil 2010] is constructed out of a $(\varphi, \Gamma)$-module of a 2-dimensional crystabeline representation of $G_{\mathbb{Q}_{p}}$ with determinant $\psi \varepsilon$, applying $\check{V}$ undoes this construction to obtain the Galois representation we started with. In particular, $\operatorname{det} \check{\boldsymbol{V}}(\Pi(\kappa(\mathfrak{n})))=\psi \varepsilon$. Moreover, it follows from [Colmez 2010, Théorème VI.6.50] that the locally algebraic vectors in $\Pi(\kappa(\mathfrak{n}))$ are isomorphic to $\Psi \otimes \operatorname{Sym}^{a} L^{2}$, which implies that

$$
\operatorname{dim}_{\kappa(\mathfrak{n})} \operatorname{Hom}_{K}(V, \Pi(\kappa(\mathfrak{n})))=\operatorname{dim}_{\kappa(\mathfrak{n})} \operatorname{Hom}_{K}\left(V, \Psi \otimes \operatorname{Sym}^{a} L^{2}\right)=1,
$$

giving part (i).

If $\Pi(\kappa(\mathfrak{n}))$ is reducible, then using the fact that (13) is nonsplit we deduce that $\Pi$ is the unique irreducible subrepresentation of $\Pi(\kappa(\mathfrak{n}))$. It follows from [Paškūnas 
2013, Lemma 12.5$]^{1}$ that the locally algebraic vectors in $\Pi$ are isomorphic to $\Psi \otimes \operatorname{Sym}^{a} L^{2}$ and the locally algebraic vectors in $\Pi(\kappa(\mathfrak{n})) / \Pi$ are zero. Thus locally algebraic vectors in $\Pi(\kappa(\mathfrak{n}))$ are isomorphic to $\Psi \otimes \operatorname{Sym}^{a} L^{2}$ and so part (i) holds. Moreover, applying $\check{V}$ to (13) we obtain an exact sequence $0 \rightarrow \delta_{2} \rightarrow$ $\check{V}(\Pi(\kappa(\mathfrak{n}))) \rightarrow \delta_{1} \rightarrow 0$. Hence, $\operatorname{det} \check{\boldsymbol{V}}(\Pi(\kappa(\mathfrak{n})))=\delta_{1} \delta_{2}=\psi \varepsilon$.

The exact sequence $0 \rightarrow \mathfrak{n} / \mathfrak{n}^{2} \rightarrow E_{\mathfrak{n}} / \mathfrak{n}^{2} \rightarrow \kappa(\mathfrak{n}) \rightarrow 0$ of $E[1 / p]$-modules gives rise to an exact sequence of admissible Banach space representations of $G$

$$
0 \rightarrow \Pi(\kappa(\mathfrak{n})) \rightarrow \Pi\left(E_{\mathfrak{n}} / \mathfrak{n}^{2}\right) \rightarrow \Pi(\kappa(\mathfrak{n}))^{\oplus d} \rightarrow 0,
$$

where $d=\operatorname{dim}_{\kappa(\mathfrak{n})} \mathfrak{n} / \mathfrak{n}^{2}$. We claim that $\operatorname{Hom}_{G}\left(\Pi, \Pi\left(E_{\mathfrak{n}} / \mathfrak{n}^{2}\right)\right)$ is one-dimensional as a $\kappa(\mathfrak{n})$-vector space. Given the claim we can deduce part (ii) by the same argument as in [Paškūnas 2015b, Corollary 4.21]. To show the claim let $\Pi^{\prime}:=\Pi(\kappa(\mathfrak{n})) / \Pi$. If $\Pi^{\prime}$ is zero then the assertion follows from (9). If $\Pi^{\prime}$ is nonzero then the reduction of the unit ball modulo $\varpi_{\kappa(\mathfrak{n})}$ is isomorphic to $\pi^{\prime}$. Since (13) is nonsplit we obtain $\operatorname{Hom}_{G}\left(\Pi^{\prime}, \Pi(\kappa(\mathfrak{n}))\right)=0$, and Lemma 2.16 implies that $\operatorname{Ext}_{G}^{1}\left(\Pi^{\prime}, \Pi(\kappa(\mathfrak{n}))\right)=0$. Hence, $\operatorname{Hom}_{G}\left(\Pi(\kappa(\mathfrak{n})), \Pi\left(E_{\mathfrak{n}} / \mathfrak{n}^{2}\right)\right) \cong \operatorname{Hom}_{G}\left(\Pi, \Pi\left(E_{\mathfrak{n}} / \mathfrak{n}^{2}\right)\right)$ and the claim follows from (9).

Let $\Theta$ be a $K$-invariant $\mathcal{O}$-lattice in $V$ and let $M(\Theta):=\operatorname{Hom}_{\mathcal{O} \| K \rrbracket}^{\text {cont }}\left(P, \Theta^{d}\right)^{d}$, where $(*)^{d}:=\operatorname{Hom}_{\mathcal{O}}(*, \mathcal{O})$. It follows from Proposition 2.8 that $\left(k \widehat{\otimes}_{E} P\right)^{\vee}$ is an admissible representation of $G$; dually, this implies that $k \widehat{\otimes}_{E} P$ is a finitely generated $\mathcal{O} \llbracket K \rrbracket-$ module. Hence, [Paškūnas 2015b, Proposition 2.15] implies that $M(\Theta)$ is a finitely generated $E$-module. We will denote by $\mathrm{m}$-Spec the set of maximal ideals of a commutative ring.

Proposition 2.19. Let $\mathfrak{a}$ be the E-annihilator of $M(\Theta)$. Then $E / \mathfrak{a}$ is reduced and $\mathcal{O}$-torsion-free. Moreover, $\mathrm{m}-\operatorname{Spec}(E / \mathfrak{a})[1 / p]$ is contained in the image of $\mathrm{m}$-Spec $R^{\psi}[1 / p]$ under $\varphi^{\sharp}:$ Spec $R^{\psi} \rightarrow \operatorname{Spec} E$.

Proof. Theorem 5.2 in [Paškūnas 2015b] implies that there is a $P$-regular $x \in E$ such that $P / x P$ is a finitely generated $\mathcal{O} \llbracket K \rrbracket$-module which is projective in $\operatorname{Mod}_{K, \psi}^{\text {pro }}(\mathcal{O})$. It follows from [Paškūnas 2015b, Lemma 2.33] that $M(\Theta)$ is Cohen-Macaulay as a module over $E$ and its Krull dimension is equal to 2. If $\mathrm{m}$ is an $E[1 / p]$-module of finite length then

$$
\operatorname{dim}_{L} \operatorname{Hom}_{K}(V, \Pi(\mathrm{m}))=\operatorname{dim}_{L} \mathrm{~m} \otimes_{E} M(\Theta),
$$

by [Paškūnas 2015b, Proposition 2.22]. Proposition 2.18 together with [Paškūnas 2015 b, Proposition 2.32] imply that $E / \mathfrak{a}$ is reduced. It is $\mathcal{O}$-torsion-free, since $M(\Theta)$ is $\mathcal{O}$-torsion-free. Let $\mathfrak{n}$ be a maximal ideal of $E[1 / p]$. Since $E$ is a quotient of $R$, $\mathfrak{n}$ lies in the image of $\mathrm{m}-\operatorname{Spec} R^{\psi}[1 / p]$ if and only if $\operatorname{det} \kappa(\mathfrak{n}) \otimes_{E} \check{\boldsymbol{V}}(P)=\psi \varepsilon$.

\footnotetext{
${ }^{1}$ The assumption $p \geq 5$ in [Paškūnas 2013, §12] is only invoked in the proof of Theorem 12.7 by appealing to Theorem 11.4. All the other arguments in that section work for all primes $p$.
} 
Proposition 2.18, (11) and (17) imply that this holds for all the maximal ideals of $(E / \mathfrak{a})[1 / p]$.

Corollary 2.20. The surjection $\varphi: E \rightarrow R^{\psi}$, given by Proposition 2.14 , induces an isomorphism $E / \mathfrak{a} \cong R^{\psi} / \varphi(\mathfrak{a})$.

Proof. Since $(E / \mathfrak{a})[1 / p]$ and $\left(R^{\psi} / \varphi(\mathfrak{a})\right)[1 / p]$ are Jacobson, Proposition 2.19 implies that $\varphi$ induces an isomorphism between $E / \mathfrak{a}$ and the image of $R^{\psi}$ in the maximal reduced quotient of $\left(R^{\psi} / \varphi(\mathfrak{a})\right)[1 / p]$. This implies that the surjection $E / \mathfrak{a} \rightarrow R^{\psi} / \varphi(\mathfrak{a})$ is injective, and hence an isomorphism.

Lemma 2.21. The E-annihilators of $\operatorname{Hom}_{K}^{\text {cont }}\left(P, V^{*}\right)$ and $M(\Theta)$ are equal.

Proof. One inclusion is trivial; the other follows from [Paškūnas 2015b, (11)], which says that $\operatorname{Hom}_{K}^{\text {cont }}\left(P, V^{*}\right)$ is naturally isomorphic to $\operatorname{Hom}_{\mathcal{O}}^{\text {cont }}(M(\Theta), L)$.

Theorem 2.22. The functor $\check{V}$ induces an isomorphism $\varphi: E \stackrel{\cong}{\longrightarrow} R^{\psi}$. Moreover, $\check{V}(P)$ is the universal deformation of $\rho$ with determinant $\psi \varepsilon$.

Proof. It follows from Corollary 2.20 and Lemma 2.21 that the kernel of $\varphi$ is contained in the $E$-annihilator of $\operatorname{Hom}_{K}^{\text {cont }}\left(P, V^{*}\right)$. It follows from Proposition 2.7 that the intersection of the annihilators as $V$ varies is zero. Hence, $\varphi$ is injective, and hence an isomorphism by Proposition 2.14. The second part is a formal consequence of the first part.

2B4. Blocks. As explained in the introduction the category $\operatorname{Mod}_{G, \psi}^{1 . a d m}(\mathcal{O})$ decomposes into a product of subcategories

$$
\operatorname{Mod}_{G, \psi}^{1 . \operatorname{adm}}(\mathcal{O}) \cong \prod_{\mathfrak{B} \in \operatorname{Irradm}_{G} / \sim} \operatorname{Mod}_{G, \psi}^{1 . \mathrm{adm}}(\mathcal{O})[\mathfrak{B}]
$$

where $\operatorname{Mod}_{G, \psi}^{1 . \operatorname{adm}}(\mathcal{O})[\mathfrak{B}]$ is the full subcategory of $\operatorname{Mod}_{G, \psi}^{1 . a d m}(\mathcal{O})$ consisting of representations with all irreducible subquotients in $\mathfrak{B}$. Dually we obtain a decomposition

$$
\mathfrak{C}(\mathcal{O}) \cong \prod_{\mathfrak{B} \in \operatorname{Irradm}_{G}^{\mathrm{adm}} / \sim} \mathfrak{C}(\mathcal{O})[\mathfrak{B}]
$$

where $M \in \mathfrak{C}(\mathcal{O})$ lies in $\mathfrak{C}(\mathcal{O})[\mathfrak{B}]$ if and only if $M^{\vee}$ lies in $\operatorname{Mod}_{G, \psi}^{\text {l.adm }}(\mathcal{O})[\mathfrak{B}]$.

For a block $\mathfrak{B}$ let $\pi_{\mathfrak{B}}=\bigoplus_{\pi \in \mathfrak{B}} \pi$, and let $\pi_{\mathfrak{B}} \hookrightarrow J_{\mathfrak{B}}$ be an injective envelope of $\pi_{\mathfrak{B}}$. Then $P_{\mathfrak{B}}:=\left(J_{\mathfrak{B}}\right)^{\vee}$ is a projective envelope of $\left(\pi_{\mathfrak{B}}\right)^{\vee}$ in $\mathfrak{C}(\mathcal{O})$. Moreover, $J_{\mathfrak{B}}$ is an injective generator of $\operatorname{Mod}_{G, \psi}^{\mathrm{l} . \mathrm{adm}}(\mathcal{O})[\mathfrak{B}]$ and $P_{\mathfrak{B}}$ is a projective generator of $\mathfrak{C}(\mathcal{O})[\mathfrak{B}]$. The ring $E_{\mathfrak{B}}:=\operatorname{End}_{\mathfrak{C}(\mathcal{O})}\left(P_{\mathfrak{B}}\right)$ carries a natural topology with respect to which it is a pseudocompact ring; see [Gabriel 1962, Chapitre IV, Proposition 13]. In addition, the functor

$$
M \mapsto \operatorname{Hom}_{\mathfrak{C}(\mathcal{O})}\left(P_{\mathfrak{B}}, M\right)
$$


induces an equivalence of categories between $\mathfrak{C}(\mathcal{O})[\mathfrak{B}]$ and the category of right pseudocompact $E_{\mathfrak{B}}$-modules; see Corollaire 1 after [Gabriel 1962, Chapitre IV, Théorème 4]. The inverse functor is given by $\mathrm{m} \mapsto \mathrm{m} \widehat{\otimes}_{E_{\mathfrak{B}}} P_{\mathfrak{B}}$, as follows from Lemmas 2.9 and 2.10 in [Paškūnas 2013]. Moreover, the center of the category of $\mathfrak{C}(\mathcal{O})[\mathfrak{B}]$, which by definition is the ring of the natural transformations of the identity functor, is naturally isomorphic to the center of the ring $E_{\mathfrak{B}}$; see Corollaire 5 after [Gabriel 1962, Chapitre IV, Théorème 4].

Let us prove Theorem 1.2, stated in the introduction. If $\mathfrak{B}$ is a block containing a supersingular representation $\pi$ then $\mathfrak{B}=\{\pi\}$ and so $\pi_{\mathfrak{B}}=\pi, P_{\mathfrak{B}}$ is a projective envelope of $\pi^{\vee}$ and $E_{\mathfrak{B}}$ coincides with the ring denoted by $E$ in the previous section. Theorem 2.22 implies that $E_{\mathfrak{B}}$ is naturally isomorphic to $R_{\rho}^{\psi}$, the quotient of the universal deformation ring of $\rho:=\check{V}\left(\pi^{\vee}\right)$ parametrizing deformations with determinant $\psi \varepsilon$. Since this ring is commutative, we deduce that the center of $\mathfrak{C}(\mathcal{O})[\mathfrak{B}]$ is naturally isomorphic to $R_{\rho}^{\psi}$. Moreover, $\check{V}\left(P_{\mathfrak{B}}\right)$ is the tautological deformation of $\rho$ to $R_{\rho}^{\psi}$; see Theorem 2.22.

If $\mathfrak{B}$ contains a generic principal series representation then $\mathfrak{B}=\left\{\pi_{1}, \pi_{2}\right\}$, where

$$
\pi_{1} \cong\left(\operatorname{Ind}_{B}^{G} \chi_{1} \otimes \chi_{2} \omega^{-1}\right)_{\mathrm{sm}}, \quad \pi_{2} \cong\left(\operatorname{Ind}_{B}^{G} \chi_{2} \otimes \chi_{1} \omega^{-1}\right)_{\mathrm{sm}},
$$

and $\chi_{1}, \chi_{2}: \mathbb{Q}_{p}^{\times} \rightarrow k^{\times}$are continuous characters such that $\chi_{1} \chi_{2}^{-1} \neq \mathbf{1}, \omega^{ \pm 1}$. Then $\pi_{\mathfrak{B}}=\pi_{1} \oplus \pi_{2}$ and so $P_{\mathfrak{B}} \cong P_{1} \oplus P_{2}$, where $P_{1}$ is a projective envelope of $\pi_{1}^{\vee}$ and $P_{2}$ is a projective envelope of $\pi_{2}^{\vee}$ in $\mathfrak{C}(\mathcal{O})$. Thus

$$
E_{\mathfrak{B}} \cong \operatorname{End}_{\mathfrak{C}(\mathcal{O})}\left(P_{1} \oplus P_{2}\right) \cong \operatorname{End}_{G_{\mathbb{Q}_{p}}}^{\text {cont }}\left(\check{V}\left(P_{1}\right) \oplus \check{\boldsymbol{V}}\left(P_{2}\right)\right),
$$

where the last isomorphism follows from [Paškūnas 2013, Lemma 8.10]. The assumption on the characters $\chi_{1}, \chi_{2}$ implies that if we consider them as representations of $G_{\mathbb{Q}_{p}}$ via the local class field theory, Ext $^{1}$-groups between them are 1-dimensional. This means there are unique up to isomorphism nonsplit extensions

$$
\rho_{1}=\left(\begin{array}{cc}
\chi_{1} & * \\
0 & \chi_{2}
\end{array}\right), \quad \rho_{2}=\left(\begin{array}{cc}
\chi_{1} & 0 \\
* & \chi_{2}
\end{array}\right) .
$$

Let $R_{1}$ be the universal deformation ring of $\rho_{1}$, let $R_{1}^{\psi}$ be the quotient of $R_{1}$ parametrizing deformations of $\rho_{1}$ with determinant $\psi \varepsilon$, and let $\rho_{1}^{\text {univ }}$ be the tautological deformation of $\rho_{1}$ to $R_{1}^{\psi}$. We define $R_{2}, R_{2}^{\psi}$ and $\rho_{2}^{\text {univ }}$ in the same way with $\rho_{2}$ instead of $\rho_{1}$. It follows from Theorem 2.22 and (21) that

$$
E_{\mathfrak{B}} \cong \operatorname{End}_{G_{\mathbb{Q}_{p}}}^{\text {cont }}\left(\rho_{1}^{\text {univ }} \oplus \rho_{2}^{\text {univ }}\right) .
$$

We have studied the right-hand side of (22) in [Paškūnas 2013, §B.1] for $p>2$ and in [Paškūnas 2015a] in general. To describe the result we need to recall the theory of determinants due to Chenevier [2014]. 
Let $\rho: G_{\mathbb{Q}_{p}} \rightarrow \mathrm{GL}_{2}(k)$ be a continuous representation. Let $\mathfrak{A}$ be the category of local artinian augmented $\mathcal{O}$-algebras with residue field $k$. Let $D^{\mathrm{ps}}: \mathfrak{A} \rightarrow$ Sets be the functor which maps $\left(A, \mathfrak{m}_{A}\right) \in \mathfrak{A}$ to the set of pairs of functions $(t, d): G_{\mathbb{Q}_{p}} \rightarrow A$ such that:

- $d: G_{\mathbb{Q}_{p}} \rightarrow A^{\times}$is a continuous group homomorphism, congruent to $\operatorname{det} \rho$ modulo $\mathfrak{m}_{A}$.

- $t: G_{\mathbb{Q}_{p}} \rightarrow A$ is a continuous function with $t(1)=2$.

- For all $g, h \in G_{\mathbb{Q}_{p}}$, the following are satisfied:

(i) $t(g) \equiv \operatorname{tr} \rho(g)\left(\bmod \mathfrak{m}_{A}\right)$.

(ii) $t(g h)=t(h g)$.

(iii) $d(g) t\left(g^{-1} h\right)-t(g) t(h)+t(g h)=0$.

The functor $D^{\mathrm{ps}}$ is prorepresented by a complete local noetherian $\mathcal{O}$-algebra $R^{\mathrm{ps}}$. Let $R^{\mathrm{ps}, \psi}$ be the quotient of $R^{\mathrm{ps}}$ parametrizing those pairs $(t, d)$ where $d=\psi \varepsilon$. Combining (22) with [Paškūnas 2015a, Propositions 3.12 and 4.3, Corollary 4.4] we obtain the following:

Theorem 2.23. Let $\mathfrak{B}=\left\{\pi_{1}, \pi_{2}\right\}$ as above and let $\rho=\chi_{1} \oplus \chi_{2}$. The center of $E_{\mathfrak{B}}$, and hence the center of the category $\mathfrak{C}(\mathcal{O})[\mathfrak{B}]$, is naturally isomorphic to $R^{\mathrm{ps}, \psi}$. Moreover, $E_{\mathfrak{B}}$ is a free $R^{\mathrm{ps}, \psi}$-module of rank 4 :

$$
E_{\mathfrak{B}} \cong\left(\begin{array}{cc}
R^{\mathrm{ps}, \psi} e_{\chi_{1}} & R^{\mathrm{ps}, \psi} \tilde{\Phi}_{12} \\
R^{\mathrm{ps}, \psi} \tilde{\Phi}_{21} & R^{\mathrm{ps}, \psi} e_{\chi_{2}}
\end{array}\right)
$$

The generators satisfy the following relations:

$$
\begin{gathered}
e_{\chi_{1}}^{2}=e_{\chi_{1}}, \quad e_{\chi_{2}}^{2}=e_{\chi_{2}}, \quad e_{\chi_{1}} e_{\chi_{2}}=e_{\chi_{2}} e_{\chi_{1}}=0, \\
e_{\chi_{1}} \tilde{\Phi}_{12}=\tilde{\Phi}_{12} e_{\chi_{2}}=\tilde{\Phi}_{12}, \quad e_{\chi_{2}} \tilde{\Phi}_{21}=\tilde{\Phi}_{21} e_{\chi_{1}}=\tilde{\Phi}_{21}, \\
e_{\chi_{2}} \tilde{\Phi}_{12}=\tilde{\Phi}_{12} e_{\chi_{1}}=e_{\chi_{1}} \tilde{\Phi}_{21}=\tilde{\Phi}_{21} e_{\chi_{2}}=\tilde{\Phi}_{12}^{2}=\tilde{\Phi}_{21}^{2}=0, \\
\tilde{\Phi}_{12} \tilde{\Phi}_{21}=c e_{\chi_{1}}, \quad \tilde{\Phi}_{21} \tilde{\Phi}_{12}=c e_{\chi_{2}} .
\end{gathered}
$$

The element $c$ is regular in $R^{\mathrm{ps}, \psi}$ and generates the reducibility ideal.

In order to state the result about the center of $\mathfrak{C}(\mathcal{O})[\mathfrak{B}]$ in a uniform way, as in Theorem 1.3, we note that if $\rho$ is an irreducible representation then mapping a deformation $\rho_{A}$ to $\left(\operatorname{tr} \rho_{A}\right.$, det $\left.\rho_{A}\right)$ induces a homomorphism of $\mathcal{O}$-algebras $R^{\mathrm{ps}} \rightarrow R_{\rho}$, which is an isomorphism by [Chenevier 2014, Theorem 2.22, Example 3.4].

For a block $\mathfrak{B}$, let $\operatorname{Ban}_{G, \psi}^{\text {adm }}(L)[\mathfrak{B}]$ be the full subcategory of $\operatorname{Ban}_{G, \psi}^{\text {adm }}(L)$ consisting of those $\Pi$ for which, for some (equivalently any) open bounded $G$-invariant lattice $\Theta$, all the irreducible subquotients of $\Theta \otimes_{\mathcal{O}} k$ lie in $\mathfrak{B}$. It is shown in 
[Paškūnas 2013, Proposition 5.36] that $\operatorname{Ban}_{G, \psi}^{\mathrm{adm}}(L)$ decomposes into a direct sum of subcategories

$$
\operatorname{Ban}_{G, \psi}^{\mathrm{adm}}(L) \cong \bigoplus_{\mathfrak{B} \in \operatorname{Irradm}_{G}^{\mathrm{adm}} / \sim} \operatorname{Ban}_{G, \psi}^{\mathrm{adm}}(L)[\mathfrak{B}] .
$$

Corollary 2.24. If $\mathfrak{B}=\{\pi\}$ with $\pi$ supersingular then let $\rho=\check{V}\left(\pi^{\vee}\right)$. If $\mathfrak{B}=$ $\left\{\pi_{1}, \pi_{2}\right\}$ with $\pi_{1}, \pi_{2}$ given by (20) then let $\rho=\check{V}\left(\pi_{1}^{\vee}\right) \oplus \check{\boldsymbol{V}}\left(\pi_{2}^{\vee}\right)=\chi_{1} \oplus \chi_{2}$. The map $\Pi \mapsto \check{V}(\Pi)$ induces a bijection between the isomorphism classes of

- absolutely irreducible nonordinary $\Pi \in \operatorname{Ban}_{G, \psi}^{\mathrm{adm}}(L)[\mathfrak{B}]$;

- absolutely irreducible $\tilde{\rho}: G_{\mathbb{Q}_{p}} \rightarrow \mathrm{GL}_{2}(L)$ such that $\operatorname{det} \tilde{\rho}=\psi \varepsilon$ and the semisimplification of the reduction modulo ఐ of a $G_{\mathbb{Q}_{p}}$-invariant $\mathcal{O}$-lattice in $\tilde{\rho}$ is isomorphic to $\rho$.

Proof. Given Theorems 1.2 and 2.23, this is proved in the same way as [Paškūnas 2013, Theorem 11.4].

If $\Pi \in \operatorname{Ban}_{G, \psi}^{\mathrm{adm}}(L)[\mathfrak{B}]$ and $\Theta$ is an open bounded $G$-invariant lattice in $\Pi$, then $\Theta / \varpi^{n}$ is an object of $\operatorname{Mod}_{G, \psi}^{1 . a d m}(\mathcal{O})[\mathfrak{B}]$ for all $n \geq 1$. Theorem 1.3 gives a natural action of $R^{\mathrm{ps}, \psi}$ on $\Theta / \varpi^{n}$ for all $n \geq 1$. Passing to the limit and inverting $p$, we obtain a natural homomorphism $R^{\mathrm{ps}, \psi}[1 / p] \rightarrow \operatorname{End}_{G}^{\text {cont }}(\Pi)$.

Corollary 2.25. Let $\mathfrak{B}$ be as in Corollary 2.24 and let $\Pi \in \operatorname{Ban}_{G, \psi}^{\operatorname{adm}}(L)[\mathfrak{B}]$ be absolutely irreducible. Then $\operatorname{tr} \check{V}(\Pi)$ is equal to the specialization of the universal pseudocharacter $t^{\mathrm{univ}}: G_{\mathbb{Q}_{p}} \rightarrow R^{\mathrm{ps}, \psi}$ at $x: R^{\mathrm{ps}, \psi} \rightarrow \operatorname{End}_{G}^{\text {cont }}(\Pi) \cong L$.

Proof. This is proved in the same way as [Paškūnas 2013, Proposition 11.3]. To carry out that proof we need to verify that $\check{\boldsymbol{V}}\left(P_{\mathfrak{B}}\right)$ is annihilated by $g^{2}-t^{\text {univ }}(g) g+\psi \varepsilon(g)$ for all $g \in G_{\mathbb{Q}_{p}}$. If $\mathfrak{B}$ contains a supersingular representation this follows from Cayley-Hamilton since $\check{V}\left(P_{\mathfrak{B}}\right)$ is the universal deformation of $\rho$ with determinant $\psi \varepsilon$, and $\operatorname{tr} \check{\boldsymbol{V}}\left(P_{\mathfrak{B}}\right)=t^{\text {univ }}$ by [Chenevier 2014, Theorem 2.22, Example 3.4]. If $\mathfrak{B}$ contains a generic principal series then $\check{\boldsymbol{V}}\left(P_{\mathfrak{B}}\right) \cong \rho_{1}^{\text {univ }} \oplus \rho_{2}^{\text {univ }}$ and the assertion follows from [Paškūnas 2015a, Proposition 3.9].

Corollary 2.26. For any $\Pi$ as in Corollary 2.24, we have $\operatorname{dim}_{L} \operatorname{Ext}_{G, \psi}^{1}(\Pi, \Pi)=3$. Proof. Let $\operatorname{Ban}_{G, \psi}^{\mathrm{adm} . \mathrm{fl}}(L)[\mathfrak{B}]$ be the full subcategory of $\operatorname{Ban}_{G, \psi}^{\mathrm{adm}}(L)[\mathfrak{B}]$ consisting of objects of finite length. It follows from [Paškūnas 2013, Theorem 4.36] that this category decomposes into a direct sum of subcategories

$$
\operatorname{Ban}_{G, \psi}^{\operatorname{adm} . f l}(L)[\mathfrak{B}] \cong \underset{n}{ } \cong \bigoplus_{\mathfrak{n}-\operatorname{Spec} R^{\mathrm{ps}, \psi}[1 / p]} \operatorname{Ban}_{G, \psi}^{\mathrm{adm} . f \mathrm{l}}(L)[\mathfrak{B}]_{\mathfrak{n}},
$$

where, for a maximal ideal $\mathfrak{n}$ of $R^{\mathrm{ps}, \psi}[1 / p]$, the direct summand $\operatorname{Ban}_{G, \psi}^{\text {adm.fl }}(L)[\mathfrak{B}]_{\mathfrak{n}}$ consists of those finite-length representations which are killed by a power of $\mathfrak{n}$. Moreover, the last part of [Paškūnas 2013, Theorem 4.36] implies that the functor 
$\Pi \mapsto \operatorname{Hom}_{\mathfrak{C}(\mathcal{O})}\left(P_{\mathfrak{B}}, \Theta^{d}\right)[1 / p]$, where $\Theta$ is any open bounded $G$-invariant lattice in $\Pi$, induces an antiequivalence of categories between $\operatorname{Ban}_{G, \psi}^{\text {adm.fl }}(L)[\mathfrak{B}]_{\mathfrak{n}}$ and the category of modules of finite length over the $\mathfrak{n}$-adic completion of $E_{\mathfrak{B}}[1 / p]$, which we denote by $\widehat{E}_{\mathfrak{B}, \mathfrak{n}}$.

Let $\tilde{\rho}=\breve{\boldsymbol{V}}(\Pi)$. Corollary 2.24 tells us that $\tilde{\rho}$ is an absolutely irreducible representation with determinant $\psi \varepsilon$. Let $\mathfrak{n}$ be the maximal ideal of $R^{\mathrm{ps}, \psi}[1 / p]$ corresponding to the pair $(\operatorname{tr} \tilde{\rho}, \operatorname{det} \tilde{\rho})$. It follows from Corollary 2.25 that $\Pi$ is annihilated by $\mathfrak{n}$ and hence lies in $\operatorname{Ban}_{G, \psi}^{\text {adm.fl }}(L)[\mathfrak{B}]_{\mathfrak{n}}$. Let $A$ be the completion of $R^{\mathrm{ps}, \psi}[1 / p]$ at $\mathfrak{n}$. In the supersingular case, $E_{\mathfrak{B}}=R^{\mathrm{ps}, \psi}=R^{\psi}$, and so $\widehat{E}_{\mathfrak{B}, \mathfrak{n}}=A$. In the generic principal series case, since $\tilde{\rho}$ is absolutely irreducible, the image of the generator of the reducible locus in $R^{\mathrm{ps}, \psi}$ in $\kappa(\mathfrak{n})$ is nonzero. It follows from the description of $E_{\mathfrak{B}}$ in Theorem 2.23 that $\widehat{E}_{\mathfrak{B}, \mathfrak{n}}$ is isomorphic to the algebra of $2 \times 2$ matrices with entries in $A$. Thus in both cases we get that $\operatorname{Ban}_{G, \psi}^{\text {adm.fl }}(L)[\mathfrak{B}]_{\mathfrak{n}}$ is antiequivalent to the category of $A$-modules of finite length, and $\Pi$ is identified with the residue field $\kappa(\mathfrak{n})$ of $A$. Hence,

$$
\operatorname{Ext}_{G, \psi}^{1}(\Pi, \Pi) \cong \operatorname{Ext}_{A}^{1}(\kappa(\mathfrak{n}), \kappa(\mathfrak{n})) .
$$

Arguing as in [Kisin 2009c, Lemma 2.3.3] we may identify $A$ with the universal deformation ring parametrizing pseudocharacters with determinant $\psi \varepsilon$ and values in local artinian $L$-algebras which lift $\operatorname{tr} \tilde{\rho}$. Since $\tilde{\rho}$ is absolutely irreducible we may further identify this ring with the quotient of the universal deformation ring of $\tilde{\rho}$ to local artinian $L$-algebras parametrizing deformations with determinant $\psi \varepsilon$. This ring is formally smooth over $L$ of dimension 3 , as $H^{2}\left(G_{\mathbb{Q}_{p}}, \operatorname{ad}^{0}(\tilde{\rho})\right) \cong$ $H^{0}\left(G_{\mathbb{Q}_{p}}, \operatorname{ad}^{0}(\tilde{\rho})(1)\right)=0$ and so the deformation problem of $\tilde{\rho}$ is unobstructed. In particular, $\operatorname{dim}_{L} \operatorname{Ext}_{A}^{1}(\kappa(\mathfrak{n}), \kappa(\mathfrak{n}))=\operatorname{dim}_{L} \mathfrak{n} A / \mathfrak{n}^{2} A=3$.

2C. The Breuil-Mézard conjecture. In this section we apply the formalism developed in [Paškūnas 2015b] to prove new cases of the Breuil-Mézard conjecture, when $p=2$. We place no restriction on $p$ in this section.

Let $\rho: G_{\mathbb{Q}_{p}} \rightarrow \mathrm{GL}_{2}(k)$ be a continuous representation which is either absolutely irreducible, in which case we let $\pi$ be a supersingular representation of $G$ such that $\boldsymbol{V}(\pi) \cong \rho$, or which is isomorphic to $\left(\begin{array}{cc}\chi_{1} & * \\ 0 & \chi_{2}\end{array}\right)$, a nonsplit extension with $\chi_{1} \chi_{2}^{-1} \neq \mathbf{1}, \omega^{ \pm 1}$, in which case we let $\pi=\left(\operatorname{Ind}_{B}^{G} \chi_{1} \otimes \chi_{2} \omega^{-1}\right)_{\mathrm{sm}}$. As before we let $R^{\psi}$ be the quotient of the universal deformation ring of $\rho$ parametrizing deformations with determinant $\psi \varepsilon$ and let $\rho^{\text {univ }}$ be the tautological deformation of $\rho$ to $R^{\psi}$.

Proposition 2.27. P satisfies the hypotheses (N0)-(N2) of [Paškūnas 2015b, §4].

Proof. (N0) says that $k \widehat{\otimes}_{R^{\psi}} P$ is of finite length and finitely generated over $\mathcal{O} \llbracket K \rrbracket$. This follows from Proposition 2.8. To verify (N1) we need to show that

$$
\operatorname{Hom}_{\mathrm{SL}_{2}\left(\mathbb{Q}_{p}\right)}\left(\mathbf{1}, P^{\vee}\right)=0 \text {. }
$$


The $\mathrm{SL}_{2}\left(\mathbb{Q}_{p}\right)$-invariants in $P^{\vee}$ are stable under the action of $G$. Since $P^{\vee}$ is an injective envelope of $\pi$, if the subspace is nonzero then it must intersect $\pi$ nontrivially. However, $\pi^{\mathrm{SL}_{2}\left(\mathbb{Q}_{p}\right)}=0$, which concludes the proof. (N2) requires $\check{V}(P)$ and $\rho^{\text {univ }}$ to be isomorphic as $R^{\psi} \llbracket G_{\mathbb{Q}_{p}} \rrbracket$-modules and this is proved in Theorem 2.22.

Recall from [Serre 2000, §V.A] that the group of $d$-dimensional cycles $\mathcal{Z}_{d}(A)$ of a noetherian ring $A$ is a free abelian group generated by $\mathfrak{p} \in \operatorname{Spec} A$ with $\operatorname{dim} A / \mathfrak{p}=d$. For $d$-dimensional cycles $\sum_{\mathfrak{p}} n_{\mathfrak{p}} \mathfrak{p}$ and $\sum_{\mathfrak{p}} m_{\mathfrak{p}} \mathfrak{p}$, we write $\sum_{\mathfrak{p}} n_{\mathfrak{p}} \mathfrak{p} \leq \sum_{\mathfrak{p}} m_{\mathfrak{p}} \mathfrak{p}$, if $n_{\mathfrak{p}} \leq m_{\mathfrak{p}}$ for all $\mathfrak{p} \in \operatorname{Spec} A$ with $\operatorname{dim} A / \mathfrak{p}=d$.

If $M$ is a finitely generated $A$-module of dimension at most $d$ then $M_{\mathfrak{p}}$ is an $A_{\mathfrak{p}}$ module of finite length, which we denote by $\ell_{A_{\mathfrak{p}}}\left(M_{\mathfrak{p}}\right)$, for all $\mathfrak{p}$ with $\operatorname{dim} A / \mathfrak{p}=d$. We note that $\ell_{A_{\mathfrak{p}}}\left(M_{\mathfrak{p}}\right)$ is nonzero only for finitely many $\mathfrak{p}$. Thus $z_{d}(M):=\sum_{\mathfrak{p}} \ell_{A_{\mathfrak{p}}}\left(M_{\mathfrak{p}}\right) \mathfrak{p}$, where the sum is taken over all $\mathfrak{p} \in \operatorname{Spec} A$ such that $\operatorname{dim} A / \mathfrak{p}=d$, is a well defined element of $\mathcal{Z}_{d}(A)$.

If $(A, \mathfrak{m})$ is a local ring then we define a Hilbert-Samuel multiplicity $e(z)$ of a cycle $z=\sum_{\mathfrak{p}} n_{\mathfrak{p}} \mathfrak{p} \in \mathcal{Z}_{d}(A)$ to equal $\sum_{\mathfrak{p}} n_{\mathfrak{p}} e(A / \mathfrak{p})$, where $e(A / \mathfrak{p})$ is the HilbertSamuel multiplicity of the ring $A / \mathfrak{p}$. If $M$ is a finitely generated $A$-module of dimension $d$ then the Hilbert-Samuel multiplicity of $M$ is equal to the HilbertSamuel multiplicity of its cycle $z_{d}(M)$; see [Serre 2000, §V.2].

If $\Theta$ is a continuous representation of $K$ on a free $\mathcal{O}$-module of finite rank, we let

$$
M(\Theta):=\left(\operatorname{Hom}_{\mathcal{O} \llbracket K \rrbracket}^{\mathrm{cont}}\left(P, \Theta^{d}\right)\right)^{d},
$$

where $(*)^{d}:=\operatorname{Hom}_{\mathcal{O}}(*, \mathcal{O})$. If $\lambda$ is a smooth representation of $K$ on an $\mathcal{O}$-torsion module of finite length then we let

$$
M(\lambda):=\left(\operatorname{Hom}_{\mathcal{O} \llbracket K \rrbracket}^{\text {cont }}\left(P, \lambda^{\vee}\right)\right)^{\vee},
$$

where the superscript $\vee$ denotes the Pontryagin dual.

Proposition 2.28. Let $\Theta$ be a continuous representation of $K$ on a free $\mathcal{O}$-module of finite rank with central character $\psi$. Then $M(\Theta)$ is a finitely generated $R^{\psi}$-module. If $M(\Theta)$ is nonzero then it is Cohen-Macaulay and has Krull dimension equal to 2. We have an equality of 1-dimensional cycles

$$
z_{1}(M(\Theta) / \varpi)=\sum_{\sigma} m_{\sigma} z_{1}(M(\sigma))
$$

where the sum is taken over all the irreducible smooth $k$-representations of $K$, and $m_{\sigma}$ denotes the multiplicity with which $\sigma$ appears as a subquotient of $\Theta \otimes_{\mathcal{O}} k$.

Moreover, $M(\sigma) \neq 0$ if and only if $\operatorname{Hom}_{K}(\sigma, \pi) \neq 0$, in which case the HilbertSamuel multiplicity of $z_{1}(M(\sigma))$ is equal to 1 .

Proof. We showed in Proposition 2.27 that $k \widehat{\otimes}_{R^{\psi}} P$ is a finitely generated $\mathcal{O} \llbracket K \rrbracket-$ module. It follows from Corollary 2.5 in [Paškūnas 2015b] that $M(\Theta)$ is a finitely 
generated $R^{\psi}$-module. The restriction of $P$ to $K$ is projective in $\operatorname{Mod}_{K, \psi}^{\text {pro }}(\mathcal{O})$ by [Paškūnas 2015b, Corollary 5.3]. Proposition 2.24 in [Paškūnas 2015b] implies that (27) holds as an equality of $(d-1)$-dimensional cycles, where $d$ is the Krull dimension of $M(\Theta)$. Theorem 5.2 in [Paškūnas 2015b] shows that there is an $x$ in the maximal ideal of $R^{\psi}$ such that we have an exact sequence $0 \rightarrow P \stackrel{x}{\rightarrow} P \rightarrow$ $P / x P \rightarrow 0$, where the restriction of $P / x P$ to $K$ is a projective envelope of $\left(\operatorname{soc}_{K} \pi\right)^{\vee}$ in $\operatorname{Mod}_{K, \psi}^{\text {pro }}(\mathcal{O})$. Lemma 2.33 in [Paškūnas 2015b] implies that $M(\Theta)$ is a CohenMacaulay module of dimension 2 and that $\varpi, x$ is a regular sequence of parameters. If $\sigma$ is an irreducible smooth $k$-representation of $K$ with central character $\psi$ then the proof of [Paškūnas 2015b, Lemma 2.33] yields an exact sequence

$$
0 \longrightarrow M(\sigma) \stackrel{x}{\longrightarrow} M(\sigma) \longrightarrow\left(\operatorname{Hom}_{\mathcal{O} \llbracket K \rrbracket}^{\text {cont }}\left(P / x P, \sigma^{\vee}\right)\right)^{\vee} \longrightarrow 0 .
$$

Since $P / x P$ is a projective envelope of $\left(\operatorname{soc}_{K} \pi\right)^{\vee}$ in $\operatorname{Mod}_{K, \psi}^{\text {pro }}(\mathcal{O})$, we deduce that $\operatorname{dim}_{k} M(\sigma) / x M(\sigma)$ is equal to $\operatorname{dim}_{k} \operatorname{Hom}_{K}(\sigma, \pi)$. If $\operatorname{Hom}_{K}(\sigma, \pi)$ is zero then Nakayama's lemma implies that $M(\sigma)=0$. If $\operatorname{Hom}_{K}(\sigma, \pi)$ is nonzero then it is a one-dimensional $k$-vector space, since the $K$-socle of $\pi$ is multiplicity free. The exact sequence $0 \rightarrow M(\sigma) \stackrel{x}{\rightarrow} M(\sigma) \rightarrow k \rightarrow 0$ implies that $M(\sigma)$ is a cyclic module, and if $\mathfrak{a}$ denotes its annihilator then $R^{\psi} / \mathfrak{a} \cong k \llbracket x \rrbracket$.

Remark 2.29. If $\rho$ is absolutely irreducible and $\left.\rho\right|_{\mathscr{Q}_{\mathbb{Q}_{p}}} \cong\left(\omega_{2}^{r+1} \oplus \omega_{2}^{p(r+1)}\right) \otimes \omega^{m}$ then

$$
\operatorname{soc}_{K} \pi \cong\left(\operatorname{Sym}^{r} k^{2} \oplus \operatorname{Sym}^{p-1-r} k^{2} \otimes \operatorname{det}^{r}\right) \otimes \operatorname{det}^{m},
$$

where $0 \leq r \leq p-1,0 \leq m \leq p-2$ and $\omega_{2}$ is the fundamental character of Serre of niveau 2; see [Breuil 2003a; 2003b]. If $\rho \cong\left(\begin{array}{cc}\chi_{1} & * \\ 0 & \chi_{2} \omega^{r+1}\end{array}\right) \otimes \omega^{m}$, where $\chi_{1}, \chi_{2}$ are unramified and $\chi_{1} \neq \chi_{2} \omega^{r+1}$ then

$$
\pi \cong\left(\operatorname{Ind}_{B}^{G} \chi_{1} \otimes \chi_{2} \omega^{r}\right)_{\mathrm{sm}} \otimes \omega^{m} \circ \text { det } .
$$

Hence, $\operatorname{soc}_{K} \pi \cong \operatorname{Sym}^{r} k^{2} \otimes \operatorname{det}^{m}$ if $0<r<p-1$ and $\operatorname{det}^{m} \oplus \operatorname{Sym}^{p-1} k^{2} \otimes \operatorname{det}^{m}$ otherwise. In particular, $\operatorname{soc}_{K} \pi$ is multiplicity free.

If $\mathfrak{n} \in \mathrm{m}$-Spec $R^{\psi}[1 / p]$ then the residue field $\kappa(\mathfrak{n})$ is a finite extension of $L$. Let $\mathcal{O}_{\kappa(\mathfrak{n})}$ be the ring of integers in $\kappa(\mathfrak{n})$. By specializing the universal deformation at $\mathfrak{n}$, we obtain a continuous representation $\rho_{\mathfrak{n}}^{\text {univ }}: G_{\mathbb{Q}_{p}} \rightarrow \mathrm{GL}_{2}\left(\mathcal{O}_{\kappa(\mathfrak{n})}\right)$, which reduces to $\rho$ modulo the maximal ideal of $\mathcal{O}_{\kappa(\mathfrak{n})}$. A $p$-adic Hodge type $(\boldsymbol{w}, \tau, \psi)$ consists of the following data: $\boldsymbol{w}=(a, b)$ is a pair of integers with $b>a, \tau: I_{\mathbb{Q}_{p}} \rightarrow \mathrm{GL}_{2}(L)$ is a representation of the inertia subgroup with an open kernel and $\psi: G_{\mathbb{Q}_{p}} \rightarrow \mathcal{O}^{\times}$is a continuous character such that $\psi \varepsilon \equiv \operatorname{det} \rho(\bmod \varpi),\left.\psi\right|_{I_{\Phi_{p}}}=\varepsilon^{a+b-1} \operatorname{det} \tau$, where $\varepsilon$ is the $p$-adic cyclotomic character. If $\rho_{\mathfrak{n}}^{\text {univ }}$ is potentially semistable then we say that it is of type $(\boldsymbol{w}, \tau, \psi)$ if its Hodge-Tate weights are equal to $\boldsymbol{w}$, the determinant 
is equal to $\psi$ and the restriction of the Weil-Deligne representation, associated to

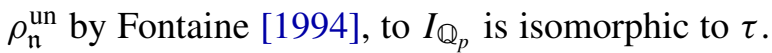

Henniart [2002] has shown the existence of a smooth irreducible representation $\sigma(\tau)$ (resp. $\left.\sigma^{\mathrm{cr}}(\tau)\right)$ of $K$ on an $L$-vector space such that if $\pi$ is a smooth absolutely irreducible infinite-dimensional representation of $G$ and $\operatorname{LL}(\pi)$ is the Weil-Deligne representation attached to $\pi$ by the classical local Langlands correspondence then $\operatorname{Hom}_{K}(\sigma(\tau), \pi) \neq 0\left(\operatorname{resp} . \operatorname{Hom}_{K}\left(\sigma^{\mathrm{cr}}(\tau), \pi\right) \neq 0\right)$ if and only if $\left.\operatorname{LL}(\pi)\right|_{I_{\mathbb{Q}_{p}}} \cong \tau$ (resp. $\left.\operatorname{LL}(\pi)\right|_{I_{\mathbb{Q}_{p}}} \cong \tau$ and the monodromy operator $N$ is 0 ). The representations $\sigma(\tau)$ and $\sigma^{\mathrm{cr}}(\tau)$ are uniquely determined if $p>2$. If $p=2$ there might be different choices; we choose one.

We let $\sigma(\boldsymbol{w}, \tau):=\sigma(\tau) \otimes \operatorname{Sym}^{b-a-1} L^{2} \otimes \operatorname{det}^{a}$. Then $\sigma(\boldsymbol{w}, \tau)$ is a finite-dimensional $L$-vector space. Since $K$ is compact and the action of $K$ on $\sigma(\boldsymbol{w}, \tau)$ is continuous, there is a $K$-invariant $\mathcal{O}$-lattice $\Theta$ in $\sigma(\boldsymbol{w}, \tau)$. Then $\Theta /(\varpi)$ is a smooth finite-length $k$-representation of $K$, and we let $\overline{\sigma(\boldsymbol{w}, \tau)}$ be its semisimplification. One may show that $\overline{\sigma(\boldsymbol{w}, \tau)}$ does not depend on the choice of a lattice. For each smooth irreducible $k$-representation $\sigma$ of $K$ we let $m_{\sigma}(\boldsymbol{w}, \tau)$ be the multiplicity with which $\sigma$ occurs in $\overline{\sigma(\boldsymbol{w}, \tau)}$. We let $\sigma^{\mathrm{cr}}(\boldsymbol{w}, \tau):=\sigma^{\mathrm{cr}}(\tau) \otimes \operatorname{Sym}^{b-a-1} L^{2} \otimes \operatorname{det}^{a}$ and let $m_{\sigma}^{\text {cr }}(\boldsymbol{w}, \tau)$ be the multiplicity of $\sigma$ in $\overline{\sigma^{\mathrm{cr}}(\boldsymbol{w}, \tau)}$. If $p=2$ then one may show that $\overline{\sigma(\boldsymbol{w}, \tau)}$ and $\overline{\sigma^{\mathrm{cr}}(\boldsymbol{w}, \tau)}$ do not depend on the choice of $\sigma(\tau)$ and $\sigma^{\mathrm{cr}}(\tau)$.

Proposition 2.30. Let $V=\sigma(\boldsymbol{w}, \tau)\left(\right.$ resp. $\left.V=\sigma^{\mathrm{cr}}(\boldsymbol{w}, \tau)\right)$ and let $\Theta$ be a $K$-invariant lattice in $V$. Then $\mathfrak{n} \in \mathrm{m}$-Spec $R^{\psi}[1 / p]$ lies in the support of $M(\Theta)$ if and only if $\rho_{\mathfrak{n}}^{\text {univ }}$ is potentially semistable (resp. potentially crystalline) of type $(\boldsymbol{w}, \tau, \psi)$. Moreover, for such $\mathfrak{n}$, we have $\operatorname{dim}_{\kappa(\mathfrak{n})} M(\Theta) \otimes_{R^{\psi}} \kappa(\mathfrak{n})=1$.

Proof. Proposition 2.22 of [Paškūnas 2015b] implies that

$$
\operatorname{dim}_{\kappa(\mathfrak{n})} M(\Theta) \otimes_{R^{\psi}} \kappa(\mathfrak{n})=\operatorname{dim}_{\kappa(\mathfrak{n})} \operatorname{Hom}_{K}(V, \Pi(\kappa(\mathfrak{n}))) .
$$

Since $V$ is a locally algebraic representation,

$$
\operatorname{Hom}_{K}(V, \Pi(\kappa(\mathfrak{n}))) \cong \operatorname{Hom}_{K}\left(V, \Pi(\kappa(\mathfrak{n}))^{\mathrm{alg}}\right),
$$

where the superscript alg denotes the subspace of locally algebraic vectors. This last subspace is nonzero if and only if $\rho_{\mathfrak{n}}^{\text {univ }}$ is potentially semistable (resp. potentially crystalline) of type $(\boldsymbol{w}, \tau, \psi)$, in which case it is one-dimensional. The argument is identical to the proof of [Paškūnas 2015b, Proposition 4.14], except that, because we assume that $\rho$ is generic, we don't have to consider the nasty cases here.

Corollary 2.31. There exists a reduced, $\mathcal{O}$-torsion-free quotient $R^{\psi}(\boldsymbol{w}, \tau)$ of $R^{\psi}$ such that a map of $\mathcal{O}$-algebras $x: R^{\psi} \rightarrow L^{\prime}$ into a finite field extension of $L$ factors through $R^{\psi}(\boldsymbol{w}, \tau)$ if and only if $\rho_{x}^{\text {univ }}$ is potentially semistable of type $(\boldsymbol{w}, \tau, \psi)$.

Moreover, if $\Theta$ is a $K$-invariant $\mathcal{O}$-lattice in $\sigma(\boldsymbol{w}, \tau)$ and $\mathfrak{a}$ is the $R^{\psi}$-annihilator of $M(\Theta)$ then $R^{\psi}(\boldsymbol{w}, \tau)=R^{\psi} / \sqrt{\mathfrak{a}}$. 
The same result holds if we consider potentially crystalline instead of potentially semistable representations with $\sigma^{\mathrm{cr}}(\boldsymbol{w}, \tau)$ instead of $\sigma(\boldsymbol{w}, \tau)$.

Proof. Since the support of $M(\Theta)$ is closed in Spec $R^{\psi}$, the assertion follows from Proposition 2.30.

Corollary 2.32. Let $\Theta$ be a $K$-invariant lattice in either $\sigma(\boldsymbol{w}, \tau)$ or $\sigma^{\mathrm{cr}}(\boldsymbol{w}, \tau)$ and let $\mathfrak{a}$ be the $R^{\psi}$-annihilator of $M(\Theta)$. Then we have equalities of cycles

$$
z_{2}\left(R^{\psi} / \mathfrak{a}\right)=z_{2}(M(\Theta)), \quad z_{1}\left(R^{\psi} /(\mathfrak{a}, \varpi)\right)=z_{1}(M(\Theta) / \varpi) .
$$

Proof. The last part of Proposition 2.30 implies that $M(\Theta)$ is generically free of rank 1. This implies the first assertion; see [Paškūnas 2015b, Lemma 2.27]. The second follows from the first combined with the fact that $\varpi$ is both $R^{\psi} / \mathfrak{a}$ - and $M(\Theta)$-regular; see Proposition 2.2.13 in [Emerton and Gee 2014].

Proposition 2.33. Let $\mathfrak{a}$ be the $R^{\psi}$-annihilator of $M(\Theta)$, where $\Theta$ is a K-invariant $\mathcal{O}$-lattice in $\sigma(\boldsymbol{w}, \tau)\left(\right.$ resp. $\left.\sigma^{\mathrm{cr}}(\boldsymbol{w}, \tau)\right)$. Then $R^{\psi} / \mathfrak{a}$ is reduced. In particular, it is equal to $R^{\psi}(\boldsymbol{w}, \tau)\left(\right.$ resp. $\left.R^{\psi, \text { cr }}(\boldsymbol{w}, \tau)\right)$.

Proof. Proposition 2.30 of [Paškūnas 2015b] together with the last part of Proposition 2.30 of the current paper says that it is enough to show that, for almost all $\mathfrak{n}$ in $\mathrm{m}-\operatorname{Spec} R^{\psi}[1 / p]$ lying in the support of $M(\Theta)$,

$$
\operatorname{dim}_{\kappa(\mathfrak{n})} \operatorname{Hom}_{K}\left(V, \Pi\left(R_{\mathfrak{n}}^{\psi} / \mathfrak{n}^{2} R_{\mathfrak{n}}^{\psi}\right)\right) \leq 2 .
$$

This amounts to checking that the subspace $\mathcal{E}$ of $\operatorname{Ext}_{G}^{1}(\Pi(\kappa(\mathfrak{n})), \Pi(\kappa(\mathfrak{n})))$ generated by the extensions of admissible unitary $\kappa(\mathfrak{n})$-Banach spaces $0 \rightarrow \Pi(\kappa(\mathfrak{n})) \rightarrow \mathrm{B} \rightarrow$ $\Pi(\kappa(\mathfrak{n})) \rightarrow 0$ such that the induced map between the subspaces of locally algebraic vectors $\mathrm{B}^{\mathrm{alg}} \rightarrow \Pi(\kappa(\mathfrak{n}))^{\text {alg }}$ is surjective, is at most one-dimensional; see the proof of [Paškūnas 2015b, Corollary 4.21].

If $\tau$ does not extend to an irreducible representation of $W_{\mathbb{Q}_{p}}$ then the proof of [Paškūnas 2015b, Theorem 4.19] carries over: the key input into that proof is that the closure of $\Pi(\kappa(\mathfrak{n}))^{\text {alg }}$ in $\Pi(\kappa(\mathfrak{n}))$ is equal to the universal unitary completion of $\Pi(\kappa(\mathfrak{n}))^{\mathrm{alg}}$ and the only case of this fact not covered by the references given in the proof of [Paškūnas 2015b, Theorem 4.19] is when $p=2$ and $\Pi(\kappa(\mathfrak{n}))^{\mathrm{alg}} \cong\left(\operatorname{Ind}_{B}^{G} \chi \otimes \chi|\cdot|^{-1}\right)_{\text {sm }} \otimes W$, where $W$ is an algebraic representation of $G$ and $\chi: \mathbb{Q}_{p}^{\times} \rightarrow \kappa(\mathfrak{n})^{\times}$is a smooth character. However, in that case it is explained in the second paragraph of the proof of [Paškūnas 2014, Proposition 6.13] how to deduce from [Paškūnas 2009, Proposition 4.2] that any $G$-invariant $\mathcal{O}$-lattice in $\Pi(\kappa(\mathfrak{n}))^{\text {alg }}$ is a finitely generated $\mathcal{O}[G]$-module, which provides the key input also in this case. We note that the assumption $p>2$ in [Paškūnas 2009, §4] is only used to apply the results of Berger, Li and Zhu; in particular, the proof of [Paškūnas 2009, Proposition 4.2] works for all $p$. 
If $\tau$ extends to an irreducible representation of $W_{\mathbb{Q}_{p}}$ then the assertion is proved by Dospinescu [2015]. Although ${ }^{2}$ the main theorem of [Dospinescu 2015] is stated under the assumption $p \geq 5$, the argument only uses that assumption if we let $\Pi=\Pi(\kappa(\mathfrak{n}))$, in which case $\operatorname{det} \check{\boldsymbol{V}}(\Pi)=\psi \varepsilon$ and $\operatorname{dim}_{L} \operatorname{Ext}_{G, \psi}^{1}(\Pi, \Pi)=3$. This is given by Corollaries 2.24 and 2.26.

Theorem 2.34. There is a finite set $\left\{\mathcal{C}_{\sigma}\right\}_{\sigma} \subset \mathcal{Z}_{1}\left(R^{\psi} / \varpi\right)$, indexed by the irreducible smooth $k$-representations $\sigma$ of $K$, such that for all p-adic Hodge types $(\boldsymbol{w}, \tau)$ we have equalities

$$
\begin{aligned}
z_{1}\left(R^{\psi}(\boldsymbol{w}, \tau) / \varpi\right) & =\sum_{\sigma} m_{\sigma}(\boldsymbol{w}, \tau) \mathcal{C}_{\sigma}, \\
z_{1}\left(R^{\psi, \mathrm{cr}}(\boldsymbol{w}, \tau) / \varpi\right) & =\sum_{\sigma} m_{\sigma}^{\mathrm{cr}}(\boldsymbol{w}, \tau) \mathcal{C}_{\sigma} .
\end{aligned}
$$

The cycle $\mathcal{C}_{\sigma}$ is nonzero if and only if $\operatorname{Hom}_{K}(\sigma, \pi) \neq 0$, in which case its HilbertSamuel multiplicity is equal to 1.

Proof. Let $\mathfrak{a}$ be the $R^{\psi}$-annihilator of $M(\Theta)$, where $\Theta$ is a $K$-invariant $\mathcal{O}$-lattice in $\sigma(\boldsymbol{w}, \tau)$. Corollary 2.31 and Proposition 2.33 imply that

$$
z_{1}\left(R^{\psi}(\boldsymbol{w}, \tau) / \varpi\right)=z_{1}\left(R^{\psi} /(\sqrt{\mathfrak{a}}, \varpi)\right)=z_{1}\left(R^{\psi} /(\mathfrak{a}, \varpi)\right) .
$$

Corollary 2.32 and Proposition 2.28 imply that

$$
z_{1}\left(R^{\psi} /(\mathfrak{a}, \varpi)\right)=\sum_{\sigma} m_{\sigma}(\boldsymbol{w}, \tau) z_{1}(M(\sigma)) .
$$

We let $\mathcal{C}_{\sigma}=z_{1}(M(\sigma))$. The proof in the potentially crystalline case is the same.

Remark 2.35. One may use a global argument to prove Proposition 2.33, without using the results of [Dospinescu 2015]. However, one needs to assume that the local residual representation can be realized as a restriction to $G_{\mathbb{Q}_{p}}$ of a global modular representation.

Let $\mathfrak{b}$ be the kernel $R^{\psi} / \mathfrak{a} \rightarrow R^{\psi} / \sqrt{\mathfrak{a}}$. Since $M(\Theta)$ is Cohen-Macaulay, $R^{\psi} / \mathfrak{a}$ is equidimensional. Thus if $\mathfrak{b}$ is nonzero then it is a 2 -dimensional $R^{\psi}$-module, and the cycle $z_{1}(\mathfrak{b} / \varpi)$ is nonzero. Since

$$
z_{1}\left(R^{\psi} /(\mathfrak{a}, \varpi)\right)=z_{1}\left(R^{\psi} /(\sqrt{\mathfrak{a}}, \varpi)\right)+z_{1}(\mathfrak{b} / \varpi),
$$

if $R^{\psi} / \mathfrak{a}$ is not reduced then we would conclude that $e\left(R^{\psi} /(\mathfrak{a}, \varpi)\right)>e\left(R^{\psi}(\boldsymbol{w}, \tau) / \varpi\right)$. Since $e\left(R^{\psi} /(\mathfrak{a}, \varpi)\right)=e(M(\Theta) / \varpi)=\sum_{\sigma} m_{\sigma}(\boldsymbol{w}, \tau) e\left(\mathcal{C}_{\sigma}\right)$, in this case we would obtain a contradiction to the Breuil-Mézard conjecture.

If the residual representation can be suitably globalized (when $p=2$ this means that it is of the form $\left.\bar{\rho}\right|_{G_{\mathbb{Q}_{p}}}$, where $\bar{\rho}$ satisfies the assumptions made in Section 3B) then a global argument gives an inequality in the opposite direction, thus allowing

\footnotetext{
${ }^{2}$ I thank G. Dospinescu for pointing this out to me.
} 
us to conclude that $R^{\psi} / \mathfrak{a}$ is reduced. If $p>2$ then such an argument is made in [Kisin 2009a, §2.3]. If $p=2$ then the same argument can be made using inequality (41) in the proof of Proposition 3.17 and the proof of Corollary 3.27.

Remark 2.36. If $R^{\square}$ is the framed deformation ring of $\rho$ and $R$ is the universal deformation ring of $\rho$ then $R^{\square} \cong R \llbracket x_{1}, x_{2}, x_{3} \rrbracket$. Thus we have a map of cycle groups

$$
f: \mathcal{Z}_{i}(R) \rightarrow \mathcal{Z}_{i+3}\left(R^{\square}\right), \quad \mathfrak{p} \mapsto \mathfrak{p} \llbracket x_{1}, x_{2}, x_{3} \rrbracket,
$$

which preserves Hilbert-Samuel multiplicities. The extra variables only keep track of a choice of basis. This implies that if $R^{\psi, \square}(\boldsymbol{w}, \tau)$ is the quotient of $R^{\square}$ parametrizing potentially semistable framed deformations of type $(\boldsymbol{w}, \tau, \psi)$ then $R^{\psi, \square}(\boldsymbol{w}, \tau) \cong R^{\psi}(\boldsymbol{w}, \tau) \llbracket x_{1}, x_{2}, x_{3} \rrbracket$, so that the cycle of $R^{\psi, \square}(\boldsymbol{w}, \tau) / \varpi$ is the image of the cycle of $R^{\psi}(\boldsymbol{w}, \tau) / \varpi$ under $f$. Using this, one may deduce a version of Theorem 2.34 for framed deformation rings.

Let $\rho=\left(\begin{array}{cc}\chi_{1} & 0 \\ 0 & \chi_{2}\end{array}\right)$, and let $R^{\square}$ be the universal framed deformation ring of $\rho$. Let $R^{\psi, \square}(\boldsymbol{w}, \tau)$ (resp. $\left.R^{\psi, \square, \text { cr }}(\boldsymbol{w}, \tau)\right)$ be the reduced, $\mathcal{O}$-torsion-free quotient of $R^{\square}$ parametrizing potentially semistable (resp. potentially crystalline) lifts of $p$-adic Hodge type $(\boldsymbol{w}, \tau, \psi)$.

Theorem 2.37. There is a subset $\left\{\mathcal{C}_{1, \sigma}, \mathcal{C}_{2, \sigma}\right\}_{\sigma}$ of $\mathcal{Z}_{4}\left(R^{\psi, \square} / \varpi\right)$ indexed by the irreducible smooth $k$-representations $\sigma$ of $K$ such that for all p-adic Hodge types $(\boldsymbol{w}, \tau)$ we have equalities

$$
\begin{aligned}
z_{4}\left(R^{\psi, \square}(\boldsymbol{w}, \tau) / \varpi\right) & =\sum_{\sigma} m_{\sigma}(\boldsymbol{w}, \tau)\left(\mathcal{C}_{1, \sigma}+\mathcal{C}_{2, \sigma}\right), \\
z_{4}\left(R^{\psi, \square, \mathrm{cr}}(\boldsymbol{w}, \tau) / \varpi\right) & =\sum_{\sigma} m_{\sigma}^{\mathrm{cr}}(\boldsymbol{w}, \tau)\left(\mathcal{C}_{1, \sigma}+\mathcal{C}_{2, \sigma}\right) .
\end{aligned}
$$

The cycle $\mathcal{C}_{1, \sigma}$ is nonzero if and only if $\operatorname{Hom}_{K}\left(\sigma,\left(\operatorname{Ind}_{B}^{G} \chi_{1} \otimes \chi_{2} \omega^{-1}\right)_{\mathrm{sm}}\right) \neq 0$, and $\mathcal{C}_{2, \sigma}$ is nonzero if and only if $\operatorname{Hom}_{K}\left(\sigma,\left(\operatorname{Ind}_{B}^{G} \chi_{2} \otimes \chi_{1} \omega^{-1}\right)_{\mathrm{sm}}\right) \neq 0$, in which case the Hilbert-Samuel multiplicity is equal to 1 .

Proof. Given Theorem 2.34, the assertion follows from Theorem 7.3 and Remark 7.4 of [Paškūnas 2015a].

The following corollary will be used in the global part of the paper.

Corollary 2.38. Assume that $p=2, \psi$ is unramified and either $\rho$ is absolutely irreducible or $\rho^{\mathrm{ss}}=\chi_{1} \oplus \chi_{2}$, with $\chi_{1} \neq \chi_{2}$. If $\boldsymbol{w}=(0,1)$ and $\tau=\mathbf{1} \oplus \mathbf{1}$ then

$$
R^{\psi, \square, \mathrm{cr}}(\boldsymbol{w}, \tau)=R^{\psi, \square}(\boldsymbol{w}, \tau) .
$$

In other words, every semistable lift of $\rho$ with Hodge-Tate weights $(0,1)$ is crystalline. 
Proof. It is enough to prove the statement when $\rho$ is nonsplit. Since if the assertion was false in the split case then by choosing a different lattice in the semistable, noncrystalline lift we would also obtain a contradiction in the nonsplit case. Since framed deformation rings are formally smooth over the nonframed ones, it is enough to prove that $R^{\psi}(\boldsymbol{w}, \tau)=R^{\psi, \text { cr }}(\boldsymbol{w}, \tau)$. By the same argument as in Remark 2.35 we see that it is enough to show that $R^{\psi}(\boldsymbol{w}, \tau) / \varpi$ and $R^{\psi, c r}(\boldsymbol{w}, \tau) / \varpi$ have the same cycles (and even the equality of Hilbert-Samuel multiplicities will suffice). Since $p=2$ there are only 2 irreducible smooth $k$-representations of $K: \mathbf{1}$ and st. The $K$-socle of $\pi$ in all the cases is isomorphic to $1 \oplus$ st, $\sigma(\boldsymbol{w}, \tau) / \varpi \cong$ st and $\sigma^{\mathrm{cr}}(\boldsymbol{w}, \tau) / \varpi \cong \mathbf{1}$. The assertion follows from Theorem 2.34.

Remark 2.39. Assume that $p=2$, let $\xi: G_{\mathbb{Q}_{p}} \rightarrow \mathcal{O}^{\times}$be unramified and congruent to $\psi$ modulo $\varpi$, and let $(\boldsymbol{w}, \tau)$ be arbitrary. It follows from Theorem 2.34, Remark 2.36, Theorem 2.37 and the proof of Corollary 2.38 that

$$
z_{4}\left(R^{\psi, \square}(\boldsymbol{w}, \tau) / \varpi\right)=\left(m_{\mathbf{1}}(\boldsymbol{w}, \tau)+m_{\mathrm{st}}(\boldsymbol{w}, \tau)\right) z_{4}\left(R^{\xi, \square}((0,1), \mathbf{1} \oplus \mathbf{1}) / \varpi\right),
$$

where the cycles live in $\mathcal{Z}_{4}\left(R^{\square}\right)$. This equality implies the equality of the respective Hilbert-Samuel multiplicities.

\section{Global part}

In the global part of the paper we let $p=2$, so that $L$ is a finite extension of $\mathbb{Q}_{2}$ with the ring integers $\mathcal{O}$ and residue field $k$.

3A. Quaternionic modular forms. We follow very closely [Kisin 2009b, §3.1]. Let $F$ be a totally real field in which 2 splits completely. Let $D$ be a quaternion algebra with center $F$, ramified at all the infinite places of $F$ and a set of finite places $\Sigma$ which does not contain any primes dividing 2 . We fix a maximal order $\mathcal{O}_{D}$ of $D$, and for each finite place $v \notin \Sigma$ we have an isomorphism $\left(\mathcal{O}_{D}\right)_{v} \cong M_{2}\left(\mathcal{O}_{F_{v}}\right)$. For each finite place $v$ of $F$ we will denote by $N(v)$ the order of the residue field at $v$, and by $\varpi_{v} \in F_{v}$ a uniformizer.

Denote by $\mathbb{A}_{F}^{f} \subset \mathbb{A}_{F}$ the finite adeles, and let $U=\prod_{v} U_{v}$ be a compact open subgroup contained in $\prod_{v}\left(\mathcal{O}_{D}\right)_{v}^{\times}$. We assume that if $v \in \Sigma$ then $U_{v}=\left(\mathcal{O}_{D}\right)_{v}^{\times}$ and if $v \mid 2$ then $U_{v}=\mathrm{GL}_{2}\left(\mathcal{O}_{F_{v}}\right)=\mathrm{GL}_{2}\left(\mathbb{Z}_{2}\right)$. Let $A$ be a topological $\mathbb{Z}_{2}$-algebra. For each $v \mid 2$, we fix a continuous representation $\sigma_{v}: U_{v} \rightarrow \operatorname{Aut}\left(W_{\sigma_{v}}\right)$ on a finite free $A$-module. Write $W_{\sigma}=\bigotimes_{v \mid 2, A} W_{\sigma_{v}}$ and denote by $\sigma: \prod_{v \mid 2} U_{v} \rightarrow \operatorname{Aut}\left(W_{\sigma}\right)$ the corresponding representation. We regard $\sigma$ as being a representation of $U$ by letting $U_{v}$ act trivially if $v \nmid 2$. Finally, assume there exists a continuous character $\psi:\left(\mathbb{A}_{F}^{f}\right)^{\times} / F^{\times} \rightarrow A^{\times}$such that, for any place $v$ of $F$, the action of $U_{v} \cap \mathcal{O}_{F_{v}}^{\times}$on $\sigma$ is given by multiplication by $\psi$. We extend the action of $U$ on $W_{\sigma}$ to $U\left(\mathbb{A}_{F}^{f}\right)^{\times}$ by letting $\left(\mathbb{A}_{F}^{f}\right)^{\times}$act via $\psi$. 
Let $S_{\sigma, \psi}(U, A)$ denote the set of continuous functions

$$
f: D^{\times} \backslash\left(D \otimes_{F} \mathbb{A}_{F}^{f}\right)^{\times} \rightarrow W_{\sigma}
$$

such that for $g \in\left(D \otimes_{F} \mathbb{A}_{F}^{f}\right)^{\times}$we have $f(g u)=\sigma(u)^{-1} f(g), u \in U$, and $f(g z)=$ $\psi^{-1}(z) f(g), z \in\left(\mathbb{A}_{F}^{f}\right)^{\times}$. If we write $\left(D \otimes_{F} \mathbb{A}_{F}^{f}\right)^{\times}=\bigsqcup_{i \in I} D^{\times} t_{i} U\left(\mathbb{A}_{F}^{f}\right)^{\times}$for some $t_{i} \in\left(D \otimes_{F} \mathbb{A}_{F}^{f}\right)^{\times}$and some finite index set $I$, then we have an isomorphism of $A$-modules

$$
S_{\sigma, \psi}(U, A) \stackrel{\cong}{\longrightarrow} \bigoplus_{i \in I} W_{\sigma}^{\left(U\left(\mathbb{A}_{F}^{f}\right)^{\times} \cap t_{i}^{-1} D^{\times} t_{i}\right) / F^{\times}}, \quad f \mapsto\left(f\left(t_{i}\right)\right)_{i \in I}
$$

Lemma 3.1. Let $U_{\max }=\prod_{v} \mathcal{O}_{D_{v}}^{\times}$, where the product is taken over all finite places of $F$. Let $t \in\left(D \otimes_{F} \mathbb{A}_{F}^{f}\right)^{\times}$. Then the group $\left(U_{\max }\left(\mathbb{A}_{F}^{f}\right)^{\times} \cap t D^{\times} t^{-1}\right) / F^{\times}$is finite and there is an integer $N$, independent of $t$, such that its order divides $N$.

Proof. This is explained in Section 7.2 of [Khare and Wintenberger 2009b]; see also [Taylor 2006, Lemma 1.1].

I thank Mark Kisin for explaining the proof of the following lemma to me.

Lemma 3.2. Let $v_{1}$ be a finite place of $F$ such that $D$ splits at $v_{1}$ and $v_{1}$ does not divide $2 N$, where $N$ is the integer defined in Lemma 3.1. Let $U=\prod_{v} U_{v}$ be a subgroup of $\left(D \otimes_{F} A_{F}^{f}\right)^{\times}$such that $U_{v}=\mathcal{O}_{D_{v}}^{\times}$if $v \neq v_{1}$ and $U_{v_{1}}$ is the subgroup of upper triangular, unipotent matrices modulo $\varpi_{v_{1}}$. Then

$$
\left(U\left(\mathbb{A}_{F}^{f}\right)^{\times} \cap t D^{\times} t^{-1}\right) / F^{\times}=1 \text { for all } t \in\left(D \otimes_{F} \mathbb{A}_{F}^{f}\right)^{\times} .
$$

Proof. Let $u \in\left(U\left(\mathbb{A}_{F}^{f}\right)^{\times} \cap t D^{\times} t^{-1}\right)$ such that $u \notin F^{\times}$. Then the $F$-subalgebra $F[u]$ of $t D t^{-1}$ is a quadratic field extension of $F$. Let $u^{\prime}$ be the conjugate of $u$ over $F$. Then $u^{\prime}=\mathrm{Nm}(u) / u$, where $\mathrm{Nm}$ is the reduced norm. Consider $w=u / u^{\prime}=$ $u^{2} / \mathrm{Nm}(u)$. Write $u=h g$ with $h \in U$ and $g \in\left(\mathbb{A}_{F}^{f}\right)^{\times}$. Then $\operatorname{Nm}(g)=g^{2}$ and so $w=u / u^{\prime}=h^{2} / \mathrm{Nm}(h)$. Thus $w$ is in $U$ and also in $t D^{\times} t^{-1}$.

Since $\left(U\left(\mathbb{A}_{F}^{f}\right)^{\times} \cap t D^{\times} t^{-1}\right) / F^{\times}$is a subgroup of $\left(U_{\max }\left(\mathbb{A}_{F}^{f}\right)^{\times} \cap t D^{\times} t^{-1}\right) / F^{\times}$, $u^{N}$ is in $F^{\times}$and hence $w^{N}=u^{N} /\left(u^{\prime}\right)^{N}=1$. Let $l$ be the prime dividing $N\left(v_{1}\right)$. Since $U_{v_{1}}$ is a pro- $l$ group and $l$ does not divide $N$, the image of $w$ under the projection $U \rightarrow U_{v_{1}}$ is equal to 1 . Since for every $v$ the map $D \rightarrow D_{v}$ is injective, we conclude that $w=1$, which implies that $u \in F$.

If (29) holds then it follows from (28) that $\sigma \mapsto S_{\sigma, \psi}(U, A)$ defines an exact functor from the category of continuous representations of $U$ on finitely generated $A$-modules, on which $U_{v}$ for $v \nmid 2$ acts trivially and $U \cap\left(\mathbb{A}_{F}^{f}\right)^{\times}$acts by $\psi$, to the category of finitely generated $A$-modules.

Let $S$ be a finite set of places of $F$ containing $\Sigma$, all the places above 2, all the infinite places and all the places $v$ for which $U_{v}$ is not maximal. Let $\mathbb{T}_{S, A}^{\text {univ }}=A\left[T_{v}, S_{v}\right]_{v \notin S}$ be a commutative polynomial ring in the indicated formal 
variables. We let $\left(D \otimes_{F} \mathbb{A}_{F}^{f}\right)^{\times}$act on the space of continuous $W_{\sigma}$-valued functions on $\left(D \otimes_{F} \mathbb{A}_{F}^{f}\right)^{\times}$by right translations, $(h f)(g):=f(g h)$. Then $S_{\sigma, \psi}(U, A)$ becomes a $\mathbb{T}_{S, A}^{\text {univ }}$-module with $S_{v}$ acting via the double coset $U_{v}\left(\begin{array}{cc}\varpi_{v} & 0 \\ 0 & \varpi_{v}\end{array}\right) U_{v}$ and $T_{v}$ acting via the double coset $U_{v}\left(\begin{array}{cc}\Phi_{v} & 0 \\ 0 & 1\end{array}\right) U_{v}$. We write $\mathbb{T}_{\sigma, \psi}(U, A)$ or $\mathbb{T}_{\sigma, \psi}(U)$ for the image of $\mathbb{T}_{S, A}^{\text {univ }}$ in the endomorphisms of $S_{\sigma, \psi}(U, A)$.

3B. Residual Galois representation. Keeping the notation of the previous section we fix an algebraic closure $\bar{F}$ of $F$ and let $G_{F, S}$ be the Galois group of the maximal extension of $F$ in $\bar{F}$ which is unramified outside $S$. We view $\psi$ as a character of $G_{F, S}$ via global class field theory, normalized so that uniformizers are mapped to geometric Frobenii. Let $\chi_{\text {cyc }}: G_{F, S} \rightarrow \mathcal{O}^{\times}$be the global 2-adic cyclotomic character. We note that $\chi_{\text {cyc }}$ is trivial modulo $\varpi$. For each place $v$ of $F$, including the infinite places, we fix an embedding $\bar{F} \hookrightarrow \bar{F}_{v}$. This induces a continuous homomorphism of Galois groups $G_{F_{v}}:=\operatorname{Gal}\left(\bar{F}_{v} / F_{v}\right) \rightarrow G_{F, S}$. We fix a continuous representation

$$
\bar{\rho}: G_{F, S} \rightarrow \mathrm{GL}_{2}(k)
$$

and assume that the following conditions hold:

- The image of $\bar{\rho}$ is nonsolvable.

- $\bar{\rho}$ is unramified at all finite places $v \nmid 2$.

- If $v \in S$ is a finite place, $v \notin \Sigma$, and $v \nmid 2$, then the eigenvalues of $\bar{\rho}\left(\right.$ Frob $\left._{v}\right)$ are distinct.

- If $v \in \Sigma$ then the eigenvalues of $\bar{\rho}\left(\right.$ Frob $\left._{v}\right)$ are equal.

- $\operatorname{det} \bar{\rho} \equiv \psi \chi_{\mathrm{cyc}}(\bmod \varpi)$.

- If $v \in S$ is a finite place, $v \notin \Sigma$, and $v \nmid 2$, then

$$
U_{v}=\left\{g \in \mathrm{GL}_{2}\left(\mathcal{O}_{F_{v}}\right): g \equiv\left(\begin{array}{cc}
1 & * \\
0 & 1
\end{array}\right)\left(\bmod \varpi_{v}\right)\right\}
$$

and at least one such $v$ does not divide $2 N$, so that the condition of Lemma 3.2 is satisfied.

3B1. Local deformation rings. We fix a basis of the underlying vector space $V_{k}$ of $\bar{\rho}$. For each $v \in S$ let $R_{v}^{\square}$ be the framed deformation ring of $\left.\bar{\rho}\right|_{G_{F_{v}}}$ and let $R_{v}^{\psi, \square}$ be the quotient of $R_{v}^{\square}$ parametrizing lifts with determinant $\psi \chi_{\text {cyc. }}$. We will now introduce some quotients of $R_{v}^{\psi, \square}$.

For $v \mid 2$ let $\tau_{v}$ be a 2-dimensional representation of the inertia group $I_{v}$ with an open kernel, and let $\boldsymbol{w}_{v}=\left(a_{v}, b_{v}\right)$ be a pair of integers with $b_{v}>a_{v}$. Let $\sigma\left(\tau_{v}\right)$ be any absolutely irreducible representation of $U_{v}=\mathrm{GL}_{2}\left(\mathbb{Z}_{2}\right)$ with the property that, for all irreducible infinite-dimensional smooth representations $\pi$ of $\mathrm{GL}_{2}\left(\mathbb{Q}_{2}\right)$, $\operatorname{Hom}_{U_{v}}\left(\sigma\left(\tau_{v}\right), \pi\right) \neq 0$ if and only if the restriction to $I_{v}$ of the Weil-Deligne 
representation $\operatorname{LL}(\pi)$ associated to $\pi$ via the local Langlands correspondence is isomorphic to $\tau$. The existence of such $\sigma\left(\tau_{v}\right)$ is shown in [Henniart 2002], where it is also shown that if $\operatorname{Hom}_{U_{v}}\left(\sigma\left(\tau_{v}\right), \pi\right) \neq 0$ then it is one-dimensional. We choose a $U_{v}$-invariant $\mathcal{O}$-lattice $\sigma\left(\tau_{v}\right)^{0}$ in $\sigma\left(\tau_{v}\right)$ and let

$$
\sigma_{v}:=\sigma\left(\tau_{v}\right)^{0} \otimes_{\mathcal{O}} \operatorname{Sym}^{b_{v}-a_{v}-1} \mathcal{O}^{2} \otimes_{\mathcal{O}} \operatorname{det}^{a_{v}} .
$$

We let $R_{v}^{\psi, \square}\left(\sigma_{v}\right)$ be the reduced, $\mathcal{O}$-flat quotient of $R_{v}^{\psi, \square}$ parametrizing potentially semistable lifts with Hodge-Tate weights $\boldsymbol{w}_{v}$ and inertial type $\tau_{v}$. This ring is denoted by $R^{\psi, \square}(\boldsymbol{w}, \tau)$ in the local part of the paper.

We similarly define $\sigma^{\mathrm{cr}}\left(\tau_{v}\right)$ by additionally requiring that $\operatorname{Hom}_{U_{v}}\left(\sigma^{\mathrm{cr}}\left(\tau_{v}\right), \pi\right) \neq 0$ if and only if the monodromy operator $N$ in $\operatorname{LL}(\pi)$ is zero and $\left.\operatorname{LL}(\pi)\right|_{I_{v}} \cong \tau_{v}$. In this case we let

$$
\sigma_{v}:=\sigma^{\mathrm{cr}}\left(\tau_{v}\right)^{0} \otimes_{\mathcal{O}} \operatorname{Sym}^{b_{v}-a_{v}-1} \mathcal{O}^{2} \otimes_{\mathcal{O}} \operatorname{det}^{a_{v}}
$$

We let $R_{v}^{\psi, \square}\left(\sigma_{v}\right)$ be the quotient of $R_{v}^{\psi, \square}$ parametrizing potentially crystalline lifts with Hodge-Tate weights $\boldsymbol{w}_{v}$ and inertial type $\tau_{v}$. This ring is denoted by $R^{\psi, \square, \mathrm{cr}}(\boldsymbol{w}, \tau)$ in the local part of the paper.

It follows either from the local part of the paper or from [Kisin 2008], where a more general result is proved, that if $R_{v}^{\psi, \square}\left(\sigma_{v}\right)$ is nonzero then it is equidimensional of Krull dimension 5. Since the residue field of $\mathbb{Z}_{2}$ has 2 elements, $\sigma\left(\tau_{v}\right)$ need not be unique (see [Henniart 2002, §§A.2.6, A.2.7]); however, the semisimplification of $\sigma\left(\tau_{v}\right)^{0} \otimes_{\mathcal{O}} k$ is the same in all cases.

If $v$ is infinite then $R_{v}^{\psi, \square}$ is a domain of Krull dimension 3 and $R_{v}^{\psi, \square}\left[\frac{1}{2}\right]$ is regular [Kisin 2009b, Proposition 2.5.6; Khare and Wintenberger 2009b, Proposition 3.1].

If $v$ is finite, $\bar{\rho}$ is unramified at $v$ and $\bar{\rho}\left(\right.$ Frob $_{v}$ ) has distinct Frobenius eigenvalues, then $R_{v}^{\psi, \square}$ has Krull dimension 4 and $R_{v}^{\psi, \square}\left[\frac{1}{2}\right]$ is regular. This follows from [Kisin 2009b, Proposition 2.5.4], where it is shown that the dimension is 4 and the irreducible components are regular. Since we assume that the eigenvalues of $\bar{\rho}\left(\right.$ Frob $\left._{v}\right)$ are distinct, $\bar{\rho}$ cannot have a lift of the form $\gamma \oplus \gamma \chi_{\text {cyc }}$. It follows from the proof of [Kisin 2009b, Proposition 2.5.4] that different irreducible components of $R_{v}^{\psi, \square}\left[\frac{1}{2}\right]$ do not intersect.

If $v$ is finite, $\psi$ and $\bar{\rho}$ are unramified at $v$ and $\bar{\rho}\left(\operatorname{Frob}_{v}\right)$ has equal eigenvalues, then for an unramified character $\gamma: G_{F_{v}} \rightarrow \mathcal{O}^{\times}$such that $\gamma^{2}=\left.\psi\right|_{G_{F_{v}}}$ we let $R_{v}^{\psi, \square}(\gamma)$ be a reduced $\mathcal{O}$-torsion-free quotient of $R_{v}^{\psi, \square}$ with the property that if $L^{\prime} / L$ is a finite extension then a map $x: R_{v}^{\psi, \square} \rightarrow L^{\prime}$ factors through $R_{v}^{\psi, \square}(\gamma)$ if and only if $V_{x}$ is isomorphic to $\left(\begin{array}{c}\gamma \chi_{\mathrm{cyc}} * \\ 0\end{array}\right)$. It follows from [Kisin 2009b, Proposition 2.5.2] via [Kisin 2009c, Proposition 2.6.6] and [Khare and Wintenberger 2009b, Theorem 3.1] that $R_{v}^{\psi, \square}(\gamma)$ is a domain of Krull dimension 4 and $R_{v}^{\psi, \square}(\gamma)\left[\frac{1}{2}\right]$ is regular. If $L$ is large enough then there are precisely two such characters, which we denote by $\gamma_{1}$ 
and $\gamma_{2}$. We let $\bar{R}_{v}^{\psi, \square}$ be the image of

$$
R_{v}^{\psi, \square} \rightarrow R_{v}^{\psi, \square}\left(\gamma_{1}\right)\left[\frac{1}{2}\right] \times R_{v}^{\psi, \square}\left(\gamma_{2}\right)\left[\frac{1}{2}\right] .
$$

Then $\bar{R}_{v}^{\psi, \square}$ is a reduced, $\mathcal{O}$-flat quotient of $R_{v}^{\psi, \square}$ such that if $L^{\prime} / L$ is a finite extension then a map $x: R_{v}^{\psi, \square} \rightarrow L^{\prime}$ factors through $\bar{R}_{v}^{\psi, \square}$ if and only if $V_{x}$ is isomorphic to $\left(\begin{array}{cc}\gamma \chi_{\mathrm{cyc}} & * \\ 0 & \gamma\end{array}\right)$ for an unramified character $\gamma$. Moreover,

$$
\bar{R}_{v}^{\psi, \square}\left[\frac{1}{2}\right] \cong R_{v}^{\psi, \square}\left(\gamma_{1}\right)\left[\frac{1}{2}\right] \times R_{v}^{\psi, \square}\left(\gamma_{2}\right)\left[\frac{1}{2}\right] .
$$

Thus $\bar{R}_{v}^{\psi, \square}\left[\frac{1}{2}\right]$ is regular and equidimensional and the Krull dimension of $\bar{R}_{v}^{\psi, \square}$ is 4 .

We let

$$
R_{S}^{\square}=\widehat{\bigotimes_{v \in S}} R_{v}^{\square}, \quad R_{S}^{\psi, \square}=\widehat{\bigotimes_{v \in S}} R_{v}^{\psi, \square}, \quad \sigma:=\widehat{\bigotimes_{v \mid 2}} \sigma_{v},
$$

and

$$
R_{S}^{\psi, \square}(\sigma):=\widehat{\bigotimes}_{v \mid 2} R_{v}^{\psi, \square}\left(\sigma_{v}\right) \widehat{\bigotimes}_{v \in \Sigma} \bar{R}_{v}^{\psi, \square} \widehat{\bigotimes}_{\substack{v \in S \backslash \Sigma \\ v \nmid 2 \infty}} R_{v}^{\psi, \square} \widehat{\bigotimes_{v \mid \infty}} R_{v}^{\psi, \square}
$$

It follows from above that $R_{S}^{\psi, \square}(\sigma)$ is equidimensional of Krull dimension equal to

$$
1+4 \sum_{v \mid 2} 1+3|\Sigma|+3\left(|S|-|\Sigma|-\sum_{v \mid 2} 1-\sum_{v \mid \infty} 1\right)+2 \sum_{v \mid \infty} 1=1+3|S| .
$$

3B2. Global deformation rings. Since $\bar{\rho}$ is assumed to have nonsolvable image, $\bar{\rho}$ is absolutely irreducible. We define $R_{F, S}^{\psi}$ to be the quotient of the universal deformation ring of $\bar{\rho}$ parametrizing deformations with determinant $\psi \chi_{\text {cyc }}$. If $Q$ is a finite set of places of $F$ disjoint from $S$ then we let $S_{Q}=S \cup Q$ and define $R_{F, S_{Q}}^{\psi}$ in the same way by viewing $\bar{\rho}$ as a representation of $G_{F, S_{Q}}$.

Denote by $R_{F, S_{Q}}^{\psi, \square}$ the complete local $\mathcal{O}$-algebra representing the functor which assigns to an artinian, augmented $\mathcal{O}$-algebra $A$ the set of isomorphism classes of tuples $\left\{V_{A}, \beta_{w}\right\}_{w \in S}$, where $V_{A}$ is a deformation of $\bar{\rho}$ to $A$ with determinant $\psi \chi_{\text {cyc }}$ and $\beta_{w}$ is a lift of a chosen basis of $V_{k}$ to a basis of $V_{A}$. The map $\left\{V_{A}, \beta_{w}\right\}_{w \in S} \mapsto\left\{V_{A}, \beta_{v}\right\}$ induces a homomorphism of $\mathcal{O}$-algebras $R_{v}^{\psi, \square} \rightarrow R_{F, S_{Q}}^{\psi, \square}$ for every $v \in S$ and hence a homomorphism of $\mathcal{O}$-algebras $R_{S}^{\psi, \square} \rightarrow R_{F, S_{Q}}^{\psi, \square}$.

3C. Patching. For each $n \geq 1$ let $Q_{n}$ be the set of places of $F$ disjoint from $S$, as in [Kisin 2009b, Lemma 3.2.2] via [Khare and Wintenberger 2009b, Proposition 5.10]. We let $Q_{0}=\varnothing$, so that $S_{Q_{n}}=S$ for $n=0$. Let $U_{Q_{n}}=\prod_{v}\left(U_{Q_{n}}\right)_{v}$ be a compact open subgroup of $\left(D \otimes_{F} \mathbb{A}_{F}^{f}\right)^{\times}$such that $\left(U_{Q_{n}}\right)_{v}=U_{v}$ for $v \notin Q_{n}$ and $\left(U_{Q_{n}}\right)_{v}$ is defined as in [Kisin 2009b, §3.1.6] for $v \in Q_{n}$.

Let $\mathfrak{m}$ be a maximal ideal of $\mathbb{T}_{S, \mathcal{O}}^{\text {univ }}$ such that the residue field is $k, T_{v}$ is mapped to $\operatorname{tr} \bar{\rho}\left(\operatorname{Frob}_{v}\right)$ and $S_{v}$ is mapped to the image of $\psi\left(\operatorname{Frob}_{v}\right)$ in $k$ for all $v \notin S$. We define 
$\mathfrak{m}_{Q_{n}}$ in $\mathbb{T}_{S_{Q_{n}}, \mathcal{O}}^{\text {univ }}$ in the same manner. Let $\sigma=\bigotimes_{v \mid 2} \sigma_{v}$, where each $\sigma_{v}$ is given by either (30) or (31). We assume that $S_{\sigma, \psi}(U, \mathcal{O})_{\mathfrak{m}} \neq 0$. Then for all $n \geq 0$ there is a surjective homomorphism of $\mathcal{O}$-algebras $R_{F, S_{Q_{n}}} \rightarrow \mathbb{T}_{\sigma, \psi}\left(U_{Q_{n}}\right)_{\mathfrak{m}_{Q_{n}}}$ such that for all $v \notin S_{Q_{n}}$ the trace of Frob $_{v}$ of the tautological $R_{F, S_{Q_{n}}}^{\psi}$-representation of $G_{F, S_{Q_{n}}}$ is mapped to $T_{v}$. Set

$$
M_{n}(\sigma)=R_{F, S_{Q_{n}}}^{\psi, \square} \otimes_{R_{F, S_{Q_{n}}}^{\psi}} S_{\sigma, \psi}\left(U_{Q_{n}}, \mathcal{O}\right)_{\mathfrak{m}_{Q_{n}}},
$$

with the convention that if $n=0$ then $Q_{n}=\varnothing, S_{Q_{n}}=S, \mathfrak{m}_{Q_{n}}=\mathfrak{m}$, so that

$$
M_{0}(\sigma)=R_{F, S}^{\psi, \square} \otimes_{R_{F, S}^{\psi}} S_{\sigma, \psi}(U, \mathcal{O})_{\mathfrak{m}} .
$$

It follows from the local-global compatibility of Jacquet-Langlands and Langlands correspondences that the action of $R_{F, S_{Q_{n}}}^{\psi, \square}$ on $M_{n}(\sigma)$ factors through the quotient

$$
R_{F, S_{Q_{n}}}^{\psi, \square}(\sigma):=R_{S}^{\psi, \square}(\sigma) \otimes_{R_{S}^{\psi, \square}} R_{F, S_{Q_{n}}}^{\psi, \square} .
$$

Let $h=\operatorname{dim}_{k} H^{1}\left(G_{F, S}, \operatorname{ad} \bar{\rho}\right)-2=\left|Q_{n}\right|$. Let $\mathfrak{a}_{\infty}$ denote the ideal of $\mathcal{O} \llbracket y_{1}, \ldots, y_{h} \rrbracket$ generated by $\left(y_{1}, \ldots, y_{h}\right)$. Since $R_{F, S_{Q_{n}}}^{\psi, \square}$ is formally smooth over $R_{F, S_{Q_{n}}}^{\psi}$ of relative dimension $j=4|S|-1$ we may choose an identification

$$
R_{F, S_{Q_{n}}}^{\psi, \square}=R_{F, S_{Q_{n}}}^{\psi} \llbracket y_{h+1}, \ldots, y_{h+j} \rrbracket
$$

and regard $M_{n}(\sigma)$ as an $\mathcal{O} \llbracket y_{1}, \ldots, y_{h+j} \rrbracket$-module. This allows us to consider $R_{F, S_{Q_{n}}}^{\psi}$ as an $R_{S}^{\psi, \square}$-algebra via the map $R_{S}^{\psi, \square} \rightarrow R_{F, S_{Q_{n}}}^{\psi, \square} /\left(y_{h+1}, \ldots, y_{h+j}\right)=R_{F, S_{Q_{n}}}^{\psi}$. We let

$$
R_{F, S_{Q_{n}}}^{\psi}(\sigma):=R_{S}^{\psi, \square}(\sigma) \otimes_{R_{S}^{\psi, \square}} R_{F, S_{Q_{n}}}^{\psi} .
$$

Let $g=2\left|Q_{n}\right|+1$ and $t=2-|S|+\left|Q_{n}\right|$ and let $\widehat{\mathbb{G}}_{m}$ be the completion of the $\mathcal{O}$-group $\mathbb{G}_{m}$ along the identity section. The patching argument as in [Khare and Wintenberger 2009b, Proposition 9.3] shows that there exist $\mathcal{O} \llbracket y_{1}, \ldots, y_{h+j} \rrbracket$-algebras $R_{\infty}^{\prime}(\sigma)$ and $R_{\infty}(\sigma)$ and an $R_{\infty}(\sigma)$-module $M_{\infty}(\sigma)$ with the following properties:

(P1) There are surjections of $\mathcal{O}$-algebras

$$
R_{S}^{\psi, \square}(\sigma) \llbracket x_{1}, \ldots, x_{g} \rrbracket \rightarrow R_{\infty}^{\prime}(\sigma) \rightarrow R_{\infty}(\sigma) .
$$

(P2) There is an isomorphism of $R_{S}^{\psi, \square}(\sigma)$-algebras

$$
R_{\infty}(\sigma) / \mathfrak{a}_{\infty} R_{\infty}(\sigma) \stackrel{\cong}{\longrightarrow} R_{F, S}^{\psi, \square}(\sigma)
$$

and an isomorphism of $R_{F, S}^{\psi, \square}(\sigma)$-modules

$$
M_{\infty}(\sigma) / \mathfrak{a}_{\infty} M_{\infty}(\sigma) \stackrel{\cong}{\longrightarrow} M_{0}(\sigma) .
$$

(P3) $M_{\infty}(\sigma)$ is finite flat over $\mathcal{O} \llbracket y_{1}, \ldots, y_{h+j} \rrbracket$. 
(P4) Spf $R_{\infty}^{\prime}(\sigma)$ is equipped with a free action of $\left(\widehat{\mathbb{G}}_{m}\right)^{t}$, and a $\left(\widehat{\mathbb{G}}_{m}\right)^{t}$-equivariant morphism $\delta: \operatorname{Spf} R_{\infty}^{\prime}(\sigma) \rightarrow\left(\widehat{\mathbb{G}}_{m}\right)^{t}$, where $\left(\widehat{\mathbb{G}}_{m}\right)^{t}$ acts on itself by the square of the identity map.

(P5) We have $\delta^{-1}(1)=\operatorname{Spf} R_{\infty}(\sigma) \subset \operatorname{Spf} R_{\infty}^{\prime}(\sigma)$, and the induced action of $\left(\widehat{\mathbb{G}}_{m}[2]\right)^{t}$ on $\operatorname{Spf} R_{\infty}(\sigma)$ lifts to $M_{\infty}(\sigma)$.

If $A$ is a local noetherian ring of dimension $d$ and $M$ is a finitely generated $A$-module, we denote by $e(M, A)$ the coefficient of $x^{d}$ in the Hilbert-Samuel polynomial of $M$ with respect to the maximal ideal of $A$, multiplied by $d$ !. In particular, $e(M, A)=0$ if $\operatorname{dim} M<\operatorname{dim} A$. If $M=A$ we abbreviate $e(M, A)$ to $e(A)$.

It follows from [Khare and Wintenberger 2009b, Proposition 2.5] that there is a complete local noetherian $\mathcal{O}$-algebra $\left(R_{\infty}^{\text {inv }}(\sigma), \mathfrak{m}_{\sigma}^{\text {inv }}\right)$ with residue field $k$ such that $\operatorname{Spf} R_{\infty}^{\text {inv }}(\sigma)=\operatorname{Spf} R_{\infty}^{\prime}(\sigma) /\left(\widehat{\mathbb{G}}_{m}\right)^{t}$. Moreover,

$$
R_{\infty}^{\prime}(\sigma)=R_{\infty}^{\mathrm{inv}}(\sigma) \widehat{\otimes}_{\mathcal{O}} \mathcal{O} \llbracket \mathbb{Z}_{2}^{t} \rrbracket \cong R_{\infty}^{\mathrm{inv}}(\sigma) \llbracket z_{1}, \ldots, z_{t} \rrbracket .
$$

This implies that

$$
\operatorname{dim} R_{\infty}^{\prime}(\sigma)=\operatorname{dim} R_{\infty}^{\mathrm{inv}}(\sigma)+t, \quad e\left(R_{\infty}^{\prime}(\sigma) / \varpi\right)=e\left(R_{\infty}^{\mathrm{inv}}(\sigma) / \varpi\right) .
$$

Lemma 3.3. There are $a_{1}, \ldots, a_{t} \in \mathfrak{m}_{\sigma}^{\text {inv }}$ such that

$$
R_{\infty}(\sigma) \cong \frac{R_{\infty}^{\mathrm{inv}}(\sigma) \llbracket z_{1} \rrbracket}{\left(\left(1+z_{1}\right)^{2}-\left(1+a_{1}\right)\right)} \otimes_{R_{\infty}^{\mathrm{inv}}(\sigma)} \cdots \otimes_{R_{\infty}^{\mathrm{inv}}(\sigma)} \frac{R_{\infty}^{\mathrm{inv}}(\sigma) \llbracket z_{t} \rrbracket}{\left(\left(1+z_{t}\right)^{2}-\left(1+a_{t}\right)\right)} .
$$

In particular, $R_{\infty}(\sigma)$ is a free $R_{\infty}^{\mathrm{inv}}(\sigma)$-module of rank $2^{t}$.

Proof. It follows from [Khare and Wintenberger 2009b, Lemma 9.4] that $\operatorname{Spf} R_{\infty}(\sigma)$ is a $\left(\widehat{\mathbb{G}}_{m}[2]\right)^{t}$-torsor over $\operatorname{Spf} R_{\infty}^{\text {inv }}(\sigma)$. The assertion follows from [SGA $3_{\text {II }} 1970$, Exposé VIII, Proposition 4.1].

Lemma 3.4. Let $\mathfrak{p} \in \operatorname{Spec} R_{\infty}^{\text {inv }}(\sigma)$. The group $\left(\widehat{\mathbb{G}}_{m}[2]\right)^{t}(\mathcal{O})$ acts transitively on the set of prime ideals of $R_{\infty}(\sigma)$ lying above $\mathfrak{p}$.

Proof. Let us write $X$ for $\operatorname{Spf} R_{\infty}(\sigma)$ and $G$ for $\left(\widehat{\mathbb{G}}_{m}[2]\right)^{t}$. The action of $G$ on $X$ induces an action of $( \pm 1)^{t}=G(\mathcal{O}) \hookrightarrow G\left(R_{\infty}(\sigma)\right)$ on $X\left(R_{\infty}(\sigma)\right)$. If $g \in G(\mathcal{O})$ we let $\phi_{g} \in X\left(R_{\infty}(\sigma)\right)$ be the image of $\left(g, \operatorname{id}_{R_{\infty}(\sigma)}\right)$. The map $g \mapsto \phi_{g}$ induces a homomorphism of groups $G(\mathcal{O}) \rightarrow \operatorname{Aut}\left(R_{\infty}(\sigma)\right)$. Explicitly, if $g=\left(\epsilon_{1}, \ldots, \epsilon_{t}\right)$, where $\epsilon_{i}$ is either 1 or -1 , then $\phi_{g}$ is $R_{\infty}^{\text {inv }}(\sigma)$-linear and maps $1+z_{i}$ to $\epsilon_{i}\left(1+z_{i}\right)$ for $1 \leq i \leq t$. It follows from (35) that $G(\mathcal{O})$ acts transitively on the set of maximal ideals of $\kappa(\mathfrak{p}) \otimes_{R_{\infty}^{\text {inv }}(\sigma)} R_{\infty}(\sigma)$.

Lemma 3.5. The support of $M_{\infty}(\sigma)$ in $\operatorname{Spec} R_{\infty}(\sigma)$ is a union of irreducible components. The Krull dimension of $\operatorname{Spec} R_{\infty}(\sigma)$ is equal to $h+j+1$. 
Proof. It follows from part (P3) above that the support of $M_{\infty}(\sigma)$ is equidimensional of dimension $h+j+1$. To prove the assertion it is enough to show that the dimension of $R_{\infty}(\sigma)$ is less than or equal to $h+j+1$. Using Lemma 3.3, (34), (P1) and (32) we deduce that $\operatorname{dim} R_{\infty}(\sigma) \leq \operatorname{dim} R_{S}^{\psi, \square}(\sigma)+g-t=3|S|+1+g-t=h+j+1$.

Lemma 3.6. $e\left(R_{\infty}^{\prime}(\sigma) / \varpi\right) \leq e\left(R_{S}^{\psi, \square}(\sigma) / \varpi\right)$.

Proof. It follows from (33) and Lemmas 3.3 and 3.5 that

$$
\operatorname{dim} R_{\infty}^{\prime}(\sigma)=\operatorname{dim} R_{\infty}(\sigma)+t=t+h+j+1=3|S|+1+g,
$$

which is also the dimension of $R_{S}^{\psi, \square}(\sigma) \llbracket x_{1}, \ldots, x_{g} \rrbracket$ by (32). The surjection in (P1) above implies that

$$
e\left(R_{\infty}^{\prime}(\sigma) / \varpi\right) \leq e\left(R_{S}^{\psi, \square}(\sigma) \llbracket x_{1}, \ldots, x_{g} \rrbracket / \varpi\right)=e\left(R_{S}^{\psi, \square}(\sigma) / \varpi\right) .
$$

Lemma 3.7. If $S_{\sigma, \psi}(U, \mathcal{O})_{\mathfrak{m}}$ is supported on a closed point $\mathfrak{n} \in \operatorname{Spec} R_{S}^{\psi, \square}(\sigma)\left[\frac{1}{2}\right]$ then the localization $R_{S}^{\psi, \square}(\sigma)_{\mathfrak{n}}$ is a regular ring.

Proof. Since the rings $R_{v}^{\square}\left[\frac{1}{2}\right]$ are regular for all $v \nmid 2$ it is enough to show that $\mathfrak{n}$ defines a regular point in Spec $R_{v}^{\psi, \square}(\sigma)$ for all $v \mid 2$. This follows from the proof of Lemma B.5.1 in [Gee and Kisin 2014]. The argument is as follows: if the point is not regular, then it must lie on the intersection of two irreducible components of Spec $R_{v}^{\psi, \square}(\sigma)$, but this would violate the weight-monodromy conjecture for $\operatorname{WD}\left(\left.\rho_{\mathfrak{n}}\right|_{G_{F_{v}}}\right)$; see [Gee and Kisin 2014] for details.

Lemma 3.8. If $S_{\sigma, \psi}(U, \mathcal{O})_{\mathfrak{m}}$ is supported on a closed point $\mathfrak{n} \in \operatorname{Spec} R_{\infty}(\sigma)\left[\frac{1}{2}\right]$ then the localization $R_{\infty}(\sigma)_{\mathfrak{n}}$ is a regular ring.

Proof. Let $\mathfrak{n}_{S}$ be the image of $\mathfrak{n}$ in Spec $R_{S}^{\psi, \square} \llbracket x_{1}, \ldots, x_{g} \rrbracket$, let $\mathfrak{n}^{\prime}$ be the image of $\mathfrak{n}$ in Spec $R_{\infty}^{\prime}(\sigma)$ via the maps in (P1), and let $\mathfrak{n}^{\text {inv }}$ be the image of $\mathfrak{n}$ in Spec $R_{\infty}^{\text {inv }}(\sigma)$ via (35). It follows from Lemma 3.7 that $R_{S}^{\psi, \square}(\sigma) \llbracket x_{1}, \ldots, x_{g} \rrbracket_{\mathfrak{n}_{S}}$ is a regular ring. If the map

$$
R_{S}^{\psi, \square}(\sigma) \llbracket x_{1}, \ldots, x_{g} \rrbracket_{\mathfrak{n}_{S}} \rightarrow R_{\infty}^{\prime}(\sigma)_{\mathfrak{n}^{\prime}}
$$

is an isomorphism, then $R_{\infty}^{\prime}(\sigma)_{\mathfrak{n}^{\prime}}$ is a regular ring. We may assume that $L$ is sufficiently large, so that using (33) we may write $\mathfrak{n}^{\prime}=\left(\mathfrak{n}^{\text {inv }}, z_{1}-a_{1}, \ldots, z_{t}-a_{t}\right)$ with $a_{i} \in \varpi \mathcal{O}$ for $1 \leq i \leq t$. The images of $z_{1}-a_{1}, \ldots, z_{t}-a_{t}$ in $\mathfrak{n}^{\prime} /\left(\mathfrak{n}^{\prime}\right)^{2}$ are linearly independent. Since

$$
R_{\infty}^{\text {inv }}(\sigma)_{\mathfrak{n}^{\text {inv }}} \cong R_{\infty}^{\prime}(\sigma)_{\mathfrak{n}^{\prime}} /\left(z_{1}-a_{1}, \ldots, z_{t}-a_{t}\right) R_{\infty}^{\prime}(\sigma)_{\mathfrak{n}^{\prime}},
$$

we deduce that $R_{\infty}^{\text {inv }}(\sigma)_{\mathfrak{n}^{\text {inv }}}$ is regular. It follows from (35) that the map

$$
R_{\infty}^{\mathrm{inv}}(\sigma)\left[\frac{1}{2}\right] \rightarrow R_{\infty}(\sigma)\left[\frac{1}{2}\right]
$$

is étale. Hence $R_{\infty}(\sigma)_{\mathfrak{n}}$ is a regular ring. 
If (36) is not an isomorphism then the dimension of the quotient must decrease. This leads to the inequality $\operatorname{dim} R_{\infty}(\sigma)_{\mathfrak{n}}<\operatorname{dim} R_{\infty}(\sigma)-1$. Since $M_{\infty}(\sigma)$ is a CohenMacaulay module, as follows from (P3), its support cannot contain embedded components, hence $\operatorname{dim} M_{\infty}(\sigma)_{\mathfrak{n}}=\operatorname{dim} M_{\infty}(\sigma)-1$. This leads to a contradiction, as $M_{\infty}(\sigma)_{\mathfrak{n}}$ is a finitely generated $R_{\infty}(\sigma)_{\mathfrak{n}}$-module.

Lemma 3.9. Let $A$ be a local noetherian ring and let $\left(x_{1}, \ldots, x_{d}\right)$ be a system of parameters of $A$. If $A$ is equidimensional then every irreducible component of $A$ contains a closed point of $\left(A /\left(x_{2}, \ldots, x_{d}\right)\right)\left[1 / x_{1}\right]$.

Proof. Let $\mathfrak{p}$ be an irreducible component of $A$. If $A /\left(\mathfrak{p}, x_{2}, \ldots, x_{d}\right)\left[1 / x_{1}\right]$ is zero then $x_{1}$ is nilpotent in $A /\left(\mathfrak{p}, x_{2}, \ldots, x_{d}\right)$. Since $\left(x_{1}, \ldots, x_{d}\right)$ is a system of parameters of $A$, we conclude that $A /\left(\mathfrak{p}, x_{2}, \ldots, x_{d}\right)$ is zero dimensional, which implies that $\operatorname{dim} A / \mathfrak{p} \leq d-1$, contradicting equidimensionality of $A$.

Lemma 3.10. There is an integer $r$, independent of $\sigma$ and the choices made in the patching process, such that for all $\mathfrak{p} \in \operatorname{Spec} R_{\infty}(\sigma)$ in the support of $M_{\infty}(\sigma)$ we have

$$
\operatorname{dim}_{\kappa(\mathfrak{p})} M_{\infty}(\sigma) \otimes_{R_{\infty}(\sigma)} \kappa(\mathfrak{p}) \geq r,
$$

with equality if $\mathfrak{p}$ is a minimal prime of $R_{\infty}(\sigma)$ in the support of $M_{\infty}(\sigma)$.

Proof. Let $\mathfrak{q}$ be a minimal prime of $R_{\infty}(\sigma)$ in the support of $M_{\infty}(\sigma)$. It is enough to show that $\operatorname{dim}_{\kappa(\mathfrak{q})} M_{\infty}(\sigma) \otimes_{R_{\infty}(\sigma)} \kappa(\mathfrak{q})$ is independent of $\mathfrak{q}$ and $\sigma$. Since

$$
M_{\infty}(\sigma) /\left(y_{1}, \ldots, y_{h+j}\right) M_{\infty}(\sigma) \cong S_{\sigma, \psi}(U, \mathcal{O})_{\mathfrak{m}}
$$

and $S_{\sigma, \psi}(U, \mathcal{O})_{\mathfrak{m}}$ is a finitely generated $\mathcal{O}$-module, $y_{1}, \ldots, y_{h+j}, \varpi$ is a system of parameters for $R_{\infty}(\sigma) / \mathfrak{q}$ and it follows from Lemma 3.9 that there is a maximal ideal $\mathfrak{n}$ of $R_{\infty}(\sigma)\left[\frac{1}{2}\right]$, contained in $V(\mathfrak{q})$, such that $S_{\sigma, \psi}(U, \mathcal{O})_{\mathfrak{n}} \neq 0$. It follows from (P3) that $M_{\infty}(\sigma)$ is a Cohen-Macaulay module. The same holds for the localization at $\mathfrak{n}$. Since $R_{\infty}(\sigma)_{\mathfrak{n}}$ is a regular ring by Lemma 3.8, a standard argument with the Auslander-Buchsbaum theorem shows that $M_{\infty}(\sigma)_{\mathfrak{n}}$ is a free $R_{\infty}(\sigma)_{\mathfrak{n}}$-module. By localizing further at $\mathfrak{q}$ we deduce that

$$
\begin{aligned}
\operatorname{dim}_{\kappa(\mathfrak{q})} M_{\infty}(\sigma) \otimes_{R_{\infty}(\sigma)} \kappa(\mathfrak{q}) & =\operatorname{dim}_{\kappa(\mathfrak{n})} M_{\infty}(\sigma) \otimes_{R_{\infty}(\sigma)} \kappa(\mathfrak{n}) \\
& =\operatorname{dim}_{\kappa(\mathfrak{n})} S_{\sigma, \psi}(U, \mathcal{O})_{\mathfrak{m}} \otimes_{R_{\infty}(\sigma)} \kappa(\mathfrak{n}) .
\end{aligned}
$$

So it is enough show that $\operatorname{dim}_{\kappa(\mathfrak{n})} S_{\sigma, \psi}(U, \mathcal{O})_{\mathfrak{m}} \otimes_{R_{\infty}(\sigma)} \kappa(\mathfrak{n})$ is independent of $\mathfrak{n}$ and $\sigma$. The action of $R_{\infty}(\sigma)$ on $S_{\sigma, \psi}(U, \mathcal{O})_{\mathfrak{m}}$ factors through the action of the Hecke algebra $\mathbb{T}_{\sigma, \psi}(U)$, which is reduced. Thus $\mathbb{T}_{\sigma, \psi}(U)\left[\frac{1}{2}\right]$ is a product of finite field extensions of $L$ and we have

$$
S_{\sigma, \psi}(U, \mathcal{O})_{\mathfrak{m}} \otimes_{R_{\infty}(\sigma)} \kappa(\mathfrak{n})=S_{\sigma, \psi}(U, \mathcal{O})_{\mathfrak{n}}=\left(S_{\sigma, \psi}(U, \mathcal{O})_{\mathfrak{m}} \otimes_{\mathcal{O}} L\right)[\mathfrak{n}]
$$


Let $\pi=\otimes_{v}^{\prime} \pi_{v}$ be the automorphic representation of $\left(D \otimes_{F} \mathbb{A}_{F}^{f}\right)^{\times}$corresponding to $f^{D} \in\left(S_{\sigma, \psi}(U, \mathcal{O})_{\mathfrak{m}} \otimes_{\mathcal{O}} L\right)[\mathfrak{n}]$. We assume that $L$ is sufficiently large. It follows from the discussion in [Kisin 2009c, §3.1.14], relating $S_{\sigma, \psi}(U, L)$ to the space of classical automorphic forms on $\left(D \otimes_{F} \mathbb{A}_{F}^{f}\right)^{\times}$, that

$$
\operatorname{dim}_{L}\left(S_{\sigma, \psi}(U, \mathcal{O})_{\mathfrak{m}} \otimes_{\mathcal{O}} L\right)[\mathfrak{n}]=\prod_{\substack{v \in S \\ v \nmid 2 \infty}} \operatorname{dim}_{L} \pi_{v}^{U_{v}} \prod_{v \mid 2} \operatorname{dim}_{L} \operatorname{Hom}_{U_{v}}\left(\sigma\left(\tau_{v}\right), \pi_{v}\right) .
$$

We claim that the right-hand side of the above equation is equal to $2^{|S \backslash(\Sigma \cup\{v \mid 2 \infty\})|}$. The claim will follow from the local-global compatibility of Langlands and JacquetLanglands correspondences. Let $\rho_{\mathfrak{n}}$ be the representation of $G_{F, S}$ corresponding to $\mathfrak{n}$, considered as a maximal ideal of $R_{F, S}^{\psi}(\sigma)\left[\frac{1}{2}\right]$. If $v \mid 2$ then the results of [Henniart 2002] imply that $\operatorname{dim}_{L} \operatorname{Hom}_{U_{v}}\left(\sigma\left(\tau_{v}\right), \pi_{v}\right)=1$. If $v \in \Sigma$ then $\pi_{v}$ is an unramified character of $D_{v}^{\times}$, and hence $\operatorname{dim}_{L} \pi_{v}^{U_{v}}=1$. If $v \in S, v \nmid 2 \infty$ and $v \notin \Sigma$ then $D$ is split at $v,\left.\bar{\rho}\right|_{G_{F_{v}}}$ is unramified and $\bar{\rho}\left(\right.$ Frob $\left._{v}\right)$ has distinct eigenvalues. This implies that $\left.\rho_{\mathfrak{n}}\right|_{G_{F_{v}}}$ is an extension of distinct tamely ramified characters $\psi_{1}, \psi_{2}$ such that $\psi_{1} \psi_{2}^{-1} \neq \chi_{\text {cyc }}^{ \pm 1}$. We deduce that $\pi_{v}$ is a tamely ramified principal series. Since $U_{v}$ is equal to the subgroup of unipotent upper-triangular matrices modulo $\varpi_{v}$ in this case, we deduce that $\operatorname{dim}_{L} \pi_{v}^{U_{v}}=2$.

Lemma 3.11. There is an integer $r$, independent of $\sigma$ and the choices made in the patching process, such that for all minimal primes $\mathfrak{p}$ of $R_{\infty}^{\mathrm{inv}}(\sigma)$ in the support of $M_{\infty}(\sigma)$ we have

$$
\operatorname{dim}_{\kappa(\mathfrak{p})} M_{\infty}(\sigma) \otimes_{R_{\infty}^{\text {inv }}(\sigma)} \kappa(\mathfrak{p})=2^{t} r .
$$

Proof. To ease the notation, let us drop $\sigma$ from it in this proof. Since $\mathfrak{p}$ is minimal, it is an associated prime and so $M_{\infty}$ will contain $R_{\infty}^{\text {inv }} / \mathfrak{p}$ as a submodule. Since $M_{\infty}$ is $\mathcal{O}$-torsion-free, this implies that the quotient field $\kappa(\mathfrak{p})$ has characteristic 0 . It follows from (35) that $R_{\infty} \otimes_{R_{\infty}^{\text {inv }}} \kappa(\mathfrak{p})$ is étale over $\kappa(\mathfrak{p})$, and so

$$
R_{\infty} \otimes_{R_{\infty}^{\text {inv }}} \kappa(\mathfrak{p}) \cong \prod_{\mathfrak{q}} \kappa(\mathfrak{q})
$$

where the product is taken over all prime ideals $\mathfrak{q}$ of $R_{\infty}$ such that $\mathfrak{q} \cap R_{\infty}^{\text {inv }}=\mathfrak{p}$. From this we get

$$
\operatorname{dim}_{\kappa(\mathfrak{p})} M_{\infty} \otimes_{R_{\infty}^{\text {inv }}} \kappa(\mathfrak{p})=\sum_{\mathfrak{q}}[\kappa(\mathfrak{q}): \kappa(\mathfrak{p})] \operatorname{dim}_{\kappa(\mathfrak{q})} M_{\infty} \otimes_{R_{\infty}} \kappa(\mathfrak{q})
$$

It follows from Lemma 3.4 and (P5) that all $\mathfrak{q}$ appearing in the sum lie in the support of $M_{\infty}$. Lemma 3.10 implies that $\operatorname{dim}_{\kappa(\mathfrak{q})} M_{\infty} \otimes_{R_{\infty}} \kappa(\mathfrak{q})=r$. Thus

$$
\operatorname{dim}_{\kappa(\mathfrak{p})} M_{\infty} \otimes_{R_{\infty}^{\text {inv }}} \kappa(\mathfrak{p})=r \operatorname{dim}_{\kappa(\mathfrak{p})} R_{\infty} \otimes_{R_{\infty}^{\text {inv }}} \kappa(\mathfrak{p})=r 2^{t}
$$

where the last equality follows from Lemma 3.3. 
Lemma 3.12. Let $A$ be a local noetherian ring, let $M, N$ be finitely generated $A$-modules of dimension $d$, and let $x \in A$ be $M$-regular and $N$-regular. If $\ell_{A_{\mathfrak{q}}}\left(M_{\mathfrak{q}}\right) \leq$ $\ell_{A_{\mathfrak{q}}}\left(N_{\mathfrak{q}}\right)$ for all $\mathfrak{q} \in \operatorname{Spec} A$ with $\operatorname{dim} A / \mathfrak{q}=d$ then

$$
e(M / x M, A / x A) \leq e(N / x N, A / x A) .
$$

If $\ell_{A_{\mathfrak{q}}}\left(M_{\mathfrak{q}}\right)=\ell_{A_{\mathfrak{q}}}\left(N_{\mathfrak{q}}\right)$ for all $\mathfrak{q} \in \operatorname{Spec} A$ with $\operatorname{dim} A / \mathfrak{q}=d$ then

$$
e(M / x M, A / x A)=e(N / x N, A / x A) .
$$

Proof. It follows from Proposition 2.2.13 in [Emerton and Gee 2014] that

$$
e(M / x M, A / x A)=\sum_{\mathfrak{q}} \ell_{A_{\mathfrak{q}}}\left(M_{\mathfrak{q}}\right) e(A /(\mathfrak{q}, x)),
$$

where the sum is taken over all primes $\mathfrak{q}$ in the support of $M$ such that $\operatorname{dim} A / \mathfrak{q}=d$. The above formula implies both assertions.

Lemma 3.13. $e\left(M_{\infty}(\sigma) / \varpi, R_{\infty}^{\text {inv }}(\sigma) / \varpi\right) \leq 2^{t} r e\left(R_{\infty}^{\text {inv }}(\sigma) / \varpi\right)$.

Proof. Let $\mathbb{T}_{\infty}^{\text {inv }}(\sigma)$ be the image of $R_{\infty}^{\text {inv }}(\sigma)$ in $\operatorname{End}_{\mathcal{O}}\left(M_{\infty}(\sigma)\right)$. Then

$$
e\left(\mathbb{T}_{\infty}^{\mathrm{inv}}(\sigma) / \varpi, R_{\infty}^{\mathrm{inv}}(\sigma) / \varpi\right) \leq e\left(R_{\infty}^{\mathrm{inv}}(\sigma) / \varpi\right) .
$$

If $\mathfrak{q}$ is a minimal prime of $R_{\infty}^{\text {inv }}(\sigma)$ in the support of $M_{\infty}(\sigma)$ then it follows from Lemma 3.11 that there are surjections $\mathbb{T}_{\infty}^{\text {inv }}(\sigma)_{\mathfrak{q}}^{\oplus 2^{t} r} \rightarrow M_{\infty}(\sigma)_{\mathfrak{q}}$. Thus $\ell\left(M_{\infty}(\sigma)_{\mathfrak{q}}\right) \leq$ $2^{t} r \ell\left(\mathbb{T}_{\infty}^{\text {inv }}(\sigma)_{\mathfrak{q}}\right)$. The assertion follows from Lemma 3.12 applied with $x=\varpi$, $M=M_{\infty}(\sigma)$ and $N=\mathbb{T}_{\infty}^{\text {inv }}(\sigma)^{\oplus 2^{t} r}$.

Lemma 3.14. If the support of $S_{\sigma, \psi}(U, \mathcal{O})_{\mathfrak{m}}$ meets every irreducible component of $R_{S}^{\psi, \square}(\sigma)$ then the following hold:

(i) $R_{S}^{\psi, \square}(\sigma) \llbracket x_{1}, \ldots, x_{g} \rrbracket \rightarrow R_{\infty}^{\prime}(\sigma)$ is an isomorphism.

(ii) $R_{\infty}^{\text {inv }}(\sigma)$ is reduced, equidimensional and $\mathcal{O}$-flat.

(iii) $R_{\infty}(\sigma)$ is reduced, equidimensional and $\mathcal{O}$-flat.

(iv) The support of $M_{\infty}(\sigma)$ meets every irreducible component of $R_{\infty}(\sigma)$.

(v) $2^{t} r e\left(R_{S}^{\psi, \square}(\sigma) / \varpi\right)=e\left(M_{\infty}(\sigma) / \varpi, R_{\infty}^{\mathrm{inv}}(\sigma) / \varpi\right)$.

Proof. Since $R_{S}^{\psi, \square}(\sigma) \llbracket x_{1}, \ldots, x_{g} \rrbracket$ is reduced and equidimensional and has the same dimension as $R_{\infty}^{\prime}(\sigma)$, to prove (i) it is enough to show that $R_{\infty}^{\prime}(\sigma)_{\mathfrak{q}} \neq 0$ for every irreducible component $V(\mathfrak{q})$ of $\operatorname{Spec} R_{S}^{\psi, \square}(\sigma) \llbracket x_{1}, \ldots, x_{g} \rrbracket$. Since the diagram

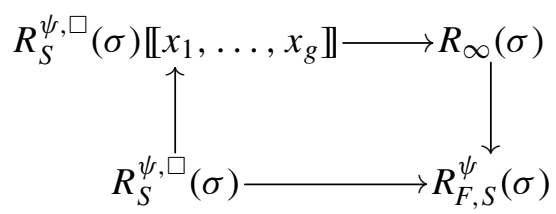


commutes and the support of $S_{\sigma, \psi}(U, \mathcal{O})_{\mathfrak{m}}$ meets every irreducible component of Spec $R_{S}^{\psi, \square}, V(\mathfrak{q})$ will contain a maximal ideal $\mathfrak{n}_{S}$ of $R_{S}^{\psi, \square}(\sigma) \llbracket x_{1}, \ldots, x_{g} \rrbracket\left[\frac{1}{2}\right]$, which lies in the support of $S_{\sigma, \psi}(U, \mathcal{O})_{\mathfrak{m}}$. It follows from the proof of Lemma 3.8 that (36) is an isomorphism in this case. Thus $R_{\infty}^{\prime}(\sigma)_{\mathfrak{q}} \neq 0$.

From part (i) we deduce that $R_{\infty}^{\prime}(\sigma)$ is reduced, equidimensional and $\mathcal{O}$-flat. It follows from (33) that the same holds for $R_{\infty}^{\text {inv }}(\sigma)$. Since $R_{\infty}(\sigma)$ is a free $R_{\infty}^{\text {inv }}(\sigma)$ module by Lemma 3.3, it is $\mathcal{O}$-flat. Hence, it is enough to show that $R_{\infty}(\sigma)\left[\frac{1}{2}\right]$ is reduced and equidimensional. It follows from Lemma 3.3 that $R_{\infty}(\sigma)\left[\frac{1}{2}\right]$ is étale over $R_{\infty}^{\text {inv }}(\sigma)\left[\frac{1}{2}\right]$, which implies the assertion. We also note that it follows from (i) that the inequality in Lemma 3.6 is an equality, and (33) implies that

$$
e\left(R_{\infty}^{\mathrm{inv}}(\sigma) / \varpi\right)=e\left(R_{S}^{\psi, \square} / \varpi\right) .
$$

It follows from our assumption that the support of $M_{\infty}(\sigma)$ meets every irreducible component of $R_{S}^{\psi, \square}(\sigma) \llbracket x_{1}, \ldots, x_{g} \rrbracket$. Part (i) and (33) imply that the support of $M_{\infty}(\sigma)$ meets every irreducible component of $R_{\infty}^{\text {inv }}(\sigma)$. It follows from Lemma 3.4 that the group $\left(\widehat{\mathbb{G}}_{m}[2]\right)^{t}(\mathcal{O})$ acts transitively on the set of irreducible components of $R_{\infty}(\sigma)$ lying above a given irreducible component of $R_{\infty}^{\text {inv }}(\sigma)$. Thus for part (iii) it is enough to show that the support of $M_{\infty}(\sigma)$ in $\operatorname{Spec} R_{\infty}(\sigma)$ is stable under the action of $\left(\widehat{\mathbb{G}}_{m}[2]\right)^{t}(\mathcal{O})$. This is given by $(\mathrm{P} 5)$ and can be proved in the same way as [Khare and Wintenberger 2009b, Lemma 9.6].

Let $V(\mathfrak{q})$ be an irreducible component of $\operatorname{Spec} R_{\infty}(\sigma)$. It follows from (iii) that the localization $R_{\infty}(\sigma)_{\mathfrak{q}}$ is a reduced artinian ring, and hence is equal to the quotient field $\kappa(\mathfrak{q})$. Thus $M_{\infty}(\sigma)_{\mathfrak{q}} \cong M_{\infty}(\sigma) \otimes_{R_{\infty}(\sigma)} \kappa(\mathfrak{q})$. It follows from Lemma 3.10 that $M_{\infty}(\sigma)_{\mathfrak{q}}$ has length $r$ as an $R_{\infty}(\sigma)_{\mathfrak{q}}$-module. By part (iv) $M_{\infty}(\sigma)$ is supported on every irreducible component of $R_{\infty}(\sigma)$, and thus the cycle of $M_{\infty}(\sigma)$ is equal to $r$ times the cycle of $R_{\infty}(\sigma)$. Since both are $\mathcal{O}$-torsion-free, we deduce that the cycle of $M_{\infty}(\sigma) / \varpi$ is equal to $r$ times the cycle of $R_{\infty}(\sigma) / \varpi$, which implies that

$$
e\left(M_{\infty}(\sigma) / \varpi, R_{\infty}^{\mathrm{inv}}(\sigma) / \varpi\right)=r e\left(R_{\infty}(\sigma) / \varpi, R_{\infty}^{\mathrm{inv}}(\sigma) / \varpi\right)=2^{t} r e\left(R_{\infty}^{\mathrm{inv}}(\sigma) / \varpi\right) .
$$

Part (v) follows from (39) and (40).

Proposition 3.15. For some $s \geq 0$ there is an isomorphism of $R_{S}^{\psi, \square}$-algebras

$$
R_{F, S}^{\psi, \square} \cong R_{S}^{\psi, \square} \llbracket x_{1}, \ldots, x_{s+|S|-1} \rrbracket /\left(f_{1}, \ldots, f_{s}\right) .
$$

Proof. The assertion follows from the proof of [Khare and Wintenberger 2009b, Proposition 4.5], where $s=\operatorname{dim}_{k} H_{\left\{L_{v}^{\perp}\right\}}^{1}\left(S,\left(\operatorname{Ad}^{0}\right)^{*}(1)\right)$ in the notation of that paper; see their Lemma 4.6 and the displayed equation above it.

Corollary 3.16. For some $s \geq 0$ there is an isomorphism of $R_{S}^{\psi, \square}(\sigma)$-algebras

$$
R_{F, S}^{\psi, \square}(\sigma) \cong R_{S}^{\psi, \square}(\sigma) \llbracket x_{1}, \ldots, x_{s+|S|-1} \rrbracket /\left(f_{1}, \ldots, f_{s}\right) .
$$


In particular, $\operatorname{dim} R_{F, S}^{\psi, \square}(\sigma) \geq 4|S|$ and $\operatorname{dim} R_{F, S}^{\psi}(\sigma) \geq 1$.

Proof. Since

$$
R_{F, S}^{\psi, \square}(\sigma) \cong R_{F, S}^{\psi, \square} \otimes_{R_{S}^{\psi, \square}} R_{S}^{\psi, \square}(\sigma)
$$

the assertion follows from Proposition 3.15. Since $\operatorname{dim} R_{S}^{\psi, \square}(\sigma)=3|S|+1$ by (32), the isomorphism implies that

$$
\operatorname{dim} R_{F, S}^{\psi, \square}(\sigma) \geq 3|S|+1+s+|S|-1-s=4|S| .
$$

Since $R_{F, S}^{\psi, \square}(\sigma)$ is formally smooth over $R_{F, S}^{\psi}(\sigma)$ of relative dimension $4|S|-1$, we conclude that $\operatorname{dim} R_{F, S}^{\psi}(\sigma) \geq 1$.

Proposition 3.17. If $S_{\sigma, \psi}(U, \mathcal{O})_{\mathfrak{m}} \neq 0$ then the following are equivalent:

(a) $2^{t} r e\left(R_{S}^{\psi, \square}(\sigma) / \varpi\right)=e\left(M_{\infty}(\sigma) / \varpi, R_{\infty}^{\text {inv }}(\sigma) / \varpi\right)$.

(b) $2^{t} r e\left(R_{S}^{\psi, \square}(\sigma) / \varpi\right) \leq e\left(M_{\infty}(\sigma) / \varpi, R_{\infty}^{\text {inv }}(\sigma) / \varpi\right)$.

(c) the support of $M_{\infty}(\sigma)$ meets every irreducible component of $R_{\infty}(\sigma)$.

(d) $R_{F, S}^{\psi}(\sigma)$ is a finitely generated $\mathcal{O}$-module of rank at least 1 and

$$
S_{\sigma, \psi}(U, \mathcal{O})_{\mathfrak{n}} \neq 0 \quad \text { for all } \mathfrak{n} \in \mathrm{m}-\operatorname{Spec} R_{F, S}^{\psi}(\sigma)\left[\frac{1}{2}\right] .
$$

In this case any representation $\rho: G_{F, S} \rightarrow \mathrm{GL}_{2}(\mathcal{O})$ corresponding to a maximal ideal of $R_{F, S}^{\psi}(\sigma)\left[\frac{1}{2}\right]$ is modular.

Proof. Lemmas 3.6 and 3.13 and (33) imply that

$$
e\left(M_{\infty}(\sigma) / \varpi, R_{\infty}^{\mathrm{inv}}(\sigma) / \varpi\right) \leq 2^{t} r e\left(R_{S}^{\psi, \square}(\sigma) / \varpi\right) .
$$

Thus (a) is equivalent to (b). Moreover, if (a) holds then the inequalities in the lemmas cited above have to be equalities. Since $R_{S}^{\psi, \square}(\sigma)$ is reduced and $\mathcal{O}$-torsionfree, we deduce that $R_{\infty}^{\prime}(\sigma) \cong R_{S}^{\psi, \square}(\sigma) \llbracket x_{1}, \ldots, x_{g} \rrbracket$. Hence, $R_{\infty}^{\prime}(\sigma)$ is reduced, equidimensional and $\mathcal{O}$-torsion-free. The isomorphism (33) implies that the same holds for $R_{\infty}^{\text {inv }}(\sigma)$, which implies that $R_{\infty}(\sigma)$ is reduced, equidimensional, and $\mathcal{O}$-torsion-free; see the proof of Lemma 3.14. Since we have assumed (a), we have

$$
2^{t} r e\left(R_{\infty}^{\mathrm{inv}}(\sigma) / \varpi\right)=e\left(M_{\infty}(\sigma) / \varpi, R_{\infty}^{\mathrm{inv}}(\sigma) / \varpi\right) .
$$

Let $V\left(\mathfrak{q}_{1}\right), \ldots, V\left(\mathfrak{q}_{m}\right)$ be the irreducible components of the support of $M_{\infty}(\sigma)$ in Spec $R_{\infty}(\sigma)$. Since $R_{\infty}(\sigma)$ is reduced, if $V(\mathfrak{q})$ is an irreducible component of Spec $R_{\infty}(\sigma)$ then $\ell\left(R_{\infty}(\sigma)_{\mathfrak{q}}\right)=1$. It follows from Lemma 3.10 that if $V(\mathfrak{q})$ is an irreducible component of Spec $R_{\infty}(\sigma)$ in the support of $M_{\infty}(\sigma)$ then $\ell\left(M_{\infty}(\sigma)_{\mathfrak{q}}\right)=r$. 
It follows from (38) that

$$
\begin{aligned}
& e\left(M_{\infty}(\sigma) / \varpi, R_{\infty}^{\mathrm{inv}}(\sigma) / \varpi\right)=r \sum_{i=1}^{m} e\left(R_{\infty}(\sigma) /\left(\varpi, \mathfrak{q}_{i}\right), R_{\infty}^{\mathrm{inv}}(\sigma) / \varpi\right), \\
& e\left(R_{\infty}(\sigma) / \varpi, R_{\infty}^{\mathrm{inv}}(\sigma) / \varpi\right)=\sum_{\mathfrak{q}} e\left(R_{\infty}(\sigma) /(\varpi, \mathfrak{q}), R_{\infty}^{\mathrm{inv}}(\sigma) / \varpi\right),
\end{aligned}
$$

where the last sum is taken over all the irreducible components $V(\mathfrak{q})$. Since $e\left(R_{\infty}(\sigma) /(\varpi, \mathfrak{q}), R_{\infty}^{\text {inv }}(\sigma) / \varpi\right) \neq 0$ we deduce from (42)-(44) that (b) implies (c). We have

$$
\begin{aligned}
R_{\infty}(\sigma) /\left(y_{1}, \ldots, y_{h+j}\right) & \cong R_{F, S}^{\psi}(\sigma), \\
M_{\infty}(\sigma) /\left(y_{1}, \ldots, y_{h+j}\right) M_{\infty}(\sigma) & \cong S_{\sigma, \psi}(U, \mathcal{O})_{\mathfrak{m}} .
\end{aligned}
$$

Thus, if $M_{\infty}(\sigma)$ is supported on the whole of $\operatorname{Spec} R_{\infty}(\sigma)$ then $S_{\sigma, \psi}(U, \mathcal{O})_{\mathfrak{m}}$ is supported on the whole of $\operatorname{Spec} R_{F, S}^{\psi}(\sigma)$. Since $S_{\sigma, \psi}(U, \mathcal{O})_{\mathfrak{m}}$ is a free $\mathcal{O}$-module of finite rank, we deduce that (c) implies (d).

If (d) holds then it follows from Corollary 3.16 that $f_{1}, \ldots, f_{s}, \varpi$ is a part of a

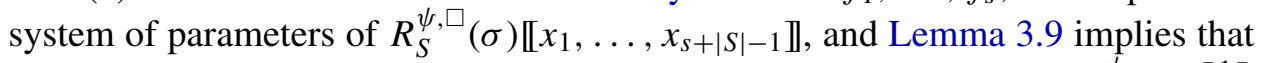
every irreducible component of that ring contains a closed point of $R_{F, S}^{\psi}(\sigma)\left[\frac{1}{2}\right]$. Since every such component is of the form $\mathfrak{q} \llbracket\left[x_{1}, \ldots, x_{s+|S|-1} \rrbracket\right.$, we deduce that every irreducible component of $R_{S}^{\psi, \square}(\sigma)$ contains a closed point of $R_{F, S}^{\psi}(\sigma)\left[\frac{1}{2}\right]$. It follows from the second part of (d) that the support of $S_{\sigma, \psi}(U, \mathcal{O})_{\mathfrak{m}}$ meets every irreducible component of $R_{S}^{\psi, \square}(\sigma)$. It follows from Lemma 3.14 that (d) implies (a). Since $S_{\sigma, \psi}(U, \mathcal{O})\left[\frac{1}{2}\right]$ is a finite-dimensional $L$-vector space, the last assertion is a direct consequence of (d).

3D. Small weights. Let $\tilde{\mathbf{1}}$ be the trivial representation of $\mathrm{GL}_{2}\left(\mathbb{Z}_{2}\right)$ on a free $\mathcal{O}$ module of rank 1 . We let st be the space of functions $f: \mathbb{P}^{1}\left(\mathbb{F}_{2}\right) \rightarrow \mathcal{O}$ such that $\sum_{x \in \mathbb{P}^{1}\left(\mathbb{F}_{2}\right)} f(x)=0$ equipped with the natural action of $\mathrm{GL}_{2}\left(\mathbb{Z}_{2}\right)$. The reduction of $\tilde{\mathbf{1}}$ modulo $\varpi$ is the trivial representation, the reduction of st modulo $\varpi$ is isomorphic to $k^{2}$, which we will also denote by st. These are the only smooth irreducible $k$-representations of $\mathrm{GL}_{2}\left(\mathbb{Z}_{2}\right)$.

The purpose of this subsection is to verify that the equivalent conditions of Proposition 3.17 hold when, for all $v \mid 2, \sigma_{v}$ is either $\tilde{\mathbf{1}}$ or $\tilde{\mathrm{st}}$, under the assumption that $\left.\bar{\rho}\right|_{G_{v}}$ does not have scalar semisimplification at any place $v \mid 2$. If $\sigma$ is the trivial representation then the result will follow from the modularity lifting theorem of [Khare and Wintenberger 2009b; Kisin 2009b]. In the general case, our assumption implies that any semistable lift of $\left.\bar{\rho}\right|_{G_{F v}}$ with Hodge-Tate weights $(0,1)$ is crystalline (see Corollary 2.38). This implies that $S_{\tilde{\mathbf{1}}, \psi}(U, \mathcal{O})_{\mathfrak{m}}$ and $S_{\sigma, \psi}(U, \mathcal{O})_{\mathfrak{m}}$ and $R_{F, S}^{\psi}(\tilde{\mathbf{1}})$ and $R_{F, S}^{\psi}(\sigma)$ coincide.

If $p>2$, the results of this section are proved in [Gee 2011] by a characteristic- $p$ argument. 
Proposition 3.18. Assume that $\psi$ is trivial on $U \cap\left(\mathbb{A}_{F}^{f}\right)^{\times}, \sigma_{v}=\tilde{\mathbf{1}}$ for all $v \mid 2$ and $\left.\bar{\rho}\right|_{G_{v}}$ does not have scalar semisimplification for any $v \mid 2$. Then $R_{F, S}^{\psi}(\sigma)$ is a finite $\mathcal{O}$-module of rank at least 1.

Proof. It follows from Lemma 2.2 in [Taylor 2003] that there is a finite solvable, totally real extension $F^{\prime}$ of $F$ such that, for all places $w$ of $F^{\prime}$ above a place $v \in S$, we have $F_{w}^{\prime}=F_{v}$, except if $v \mid 2$ and $\left.\bar{\rho}\right|_{G_{v}}$ is unramified, in which case $F_{w}^{\prime}$ is an unramified extension of $\mathbb{Q}_{2}$ and $\left.\bar{\rho}\right|_{G_{F_{w}^{\prime}}}$ is trivial. Let $S^{\prime}$ be the places of $F^{\prime}$ above the places $S$ of $F$. By changing $F$ by $F^{\prime}$ we are in position to apply Proposition 9.3 of [Khare and Wintenberger 2009b], part (II) of which says that the ring $R_{F^{\prime}, S^{\prime}}^{\psi}(\sigma)$ is a finite $\mathcal{O}$-module. We now argue as in the last paragraph of the proof of Theorem 10.1 of [Khare and Wintenberger 2009b]. The restriction to $G_{F^{\prime}, S^{\prime}}$ induces a map between the deformation functors and hence a homomorphism $R_{F^{\prime}, S^{\prime}}^{\psi}(\sigma) \rightarrow R_{F, S}^{\psi}(\sigma)$. Let $\rho_{F, S}^{\psi}: G_{F, S} \rightarrow \mathrm{GL}_{2}\left(R_{F, S}^{\psi}(\sigma)\right)$ be the universal deformation. Since $R_{F^{\prime}, S^{\prime}}^{\psi}(\sigma) / \varpi$ is finite, the image of $G_{F^{\prime}, S^{\prime}}$ in $\operatorname{GL}_{2}\left(R_{F, S}^{\psi}(\sigma) / \varpi\right)$ under $\rho_{F, S}^{\psi}$ is a finite group. Since $F^{\prime} / F$ is finite the image of $G_{F, S}$ in $\operatorname{GL}_{2}\left(R_{F, S}^{\psi}(\sigma) / \varpi\right)$ is a finite group. Lemma 3.6 in [Khare and Wintenberger 2009a] implies that $R_{F, S}^{\psi}(\sigma) / \varpi$ is finite. Since $\operatorname{dim} R_{F, S}^{\psi}(\sigma) \geq 1$ by Corollary 3.16, we conclude that $\operatorname{dim} R_{F, S}^{\psi}(\sigma)=1$ and $\varpi$ is a system of parameters for $R_{F, S}^{\psi}(\sigma)$, which implies that $R_{F, S}^{\psi}(\sigma)$ is a finite $\mathcal{O}$-module of rank at least 1 .

Corollary 3.19. Assume that $\psi$ is trivial on $U \cap\left(\mathbb{A}_{F}^{f}\right)^{\times}, \sigma_{v}=\tilde{\mathbf{1}}$ for all $v \mid 2$ and $\left.\bar{\rho}\right|_{G_{v}}$ does not have scalar semisimplification for any $v \mid 2$. If $S_{\sigma, \psi}(U, \mathcal{O})_{\mathfrak{m}} \neq 0$ then the equivalent conditions of Proposition 3.17 hold.

Proof. Since $S_{\sigma, \psi}(U, \mathcal{O})_{\mathfrak{m}}$ is nonzero and $\mathcal{O}$-torsion-free, there is a maximal ideal $\mathfrak{n}$ of $R_{F, S}^{\psi}\left[\frac{1}{2}\right]$ such that $S_{\sigma, \psi}(U, \mathcal{O})_{\mathfrak{n}} \neq 0$. This implies that $\bar{\rho}$ satisfies hypotheses $(\alpha)$ and $(\beta)$ made in Section 8.2 of [Khare and Wintenberger 2009b].

Let $\mathfrak{n}$ be any maximal ideal of $R_{F, S}^{\psi, \square}(\sigma)\left[\frac{1}{2}\right]$, and let $\rho_{\mathfrak{n}}$ be the corresponding representation of $G_{F, S}$. It follows from Theorem 9.7 in [Khare and Wintenberger 2009b] or Theorem 3.3.5 of [Kisin 2009b] that there is a Hilbert eigenform $f$ over $F$ such that $\rho_{\mathfrak{n}} \cong \rho_{f}$. Let $\pi=\bigotimes_{v}^{\prime} \pi_{v}$ be the corresponding automorphic representation of $\mathrm{GL}_{2}\left(\mathbb{A}_{F}^{f}\right)$. If $v$ is a finite place, where $D$ ramifies, then, because of the way we have set up our deformation problem, $\left.\rho_{\mathfrak{n}}\right|_{G_{F_{v}}}$ is isomorphic to $\left(\begin{array}{c}\gamma_{v} \chi_{\mathrm{cyc}} * \\ 0\end{array} \gamma_{v}\right)$, where $\gamma_{v}$ is an unramified character. The restriction of the 2-adic cyclotomic character to $G_{F_{v}}$ is an unramified character which sends the arithmetic Frobenius to $q_{v} \in \mathbb{Z}_{2}^{\times}$. Since $\rho_{\mathfrak{n}}$ arises from a Hilbert modular form, the representation $\left.\rho_{\mathfrak{n}}\right|_{G_{F_{v}}}$ cannot be split, as in this case we would obtain a contradiction to the purity of $\rho_{\mathfrak{n}}$; see [Blasius 2006, §2.2]. Hence, $\left.\rho_{\mathfrak{n}}\right|_{G_{F_{v}}}$ is nonsplit, and this implies that $\pi_{v}$ is a twist of the Steinberg representation by an unramified character, at all $v$, where $D$ is ramified. By Jacquet-Langlands correspondence there is an eigenform $f^{D} \in S_{\sigma, \psi}(U, \mathcal{O})_{\mathfrak{m}}$ 
with the same Hecke eigenvalues as $f$. This implies that $S_{\sigma, \psi}(U, \mathcal{O})_{\mathfrak{m}}$ is supported on $\mathfrak{n}$. Proposition 3.18 implies that part (d) of Proposition 3.17 holds.

Lemma 3.20. Fix a place $w$ of $F$ above 2. Let $\sigma$ and $\sigma^{\prime}$ be such that for all $v \mid 2, v \neq w$, we have $\sigma_{v}=\sigma_{v}^{\prime}$, which is equal to either $\tilde{\mathbf{1}}$ or $\tilde{\mathrm{st}}$, and $\sigma_{w}=\tilde{\mathbf{1}}$ and $\sigma_{w}^{\prime}=\tilde{\text { st. }}$. Assume that $\psi$ is trivial on $U \cap\left(\mathbb{A}_{F}^{f}\right)^{\times}$, and $\left.\bar{\rho}\right|_{G_{F}}$ does not have scalar semisimplification. Then the rings $R_{F, S}^{\psi}(\sigma)$ and $R_{F, S}^{\psi}\left(\sigma^{\prime}\right)$ are equal. Moreover, if $\mathfrak{n}$ is a maximal ideal of $R_{F, S}^{\psi}(\sigma)\left[\frac{1}{2}\right]$ then $S_{\sigma, \psi}(U, \mathcal{O})_{\mathfrak{m}}$ is supported on $\mathfrak{n}$ if and only if $S_{\sigma^{\prime}, \psi}(U, \mathcal{O})_{\mathfrak{m}}$ is supported on $\mathfrak{n}$.

Proof. The ring $R_{w}^{\psi, \square}(\tilde{\mathbf{1}})$ parametrizes crystalline lifts of $\left.\bar{\rho}\right|_{G_{F_{w}}}$ with Hodge-Tate weights $(0,1)$. The ring $R_{w}^{\psi, \square}$ ( (st) parametrizes semistable lifts of $\left.\bar{\rho}\right|_{G_{F_{w}}}$ with HodgeTate weights $(0,1)$. Since both rings are reduced and $\mathcal{O}$-torsion-free, we have a surjection $R_{w}^{\psi, \square}(\tilde{\mathrm{st}}) \rightarrow R_{w}^{\psi, \square}(\tilde{\mathbf{1}})$. The assumption that $\left.\bar{\rho}\right|_{G_{F_{w}}}$ does not have scalar semisimplification implies that every such semistable lift is automatically crystalline, hence the map is an isomorphism. This implies that the global deformation rings are equal; see Corollary 2.38.

We will deduce the second assertion from the Jacquet-Langlands correspondence and the compatibility of local and global Langlands correspondence. Let $\tau$ be either $\sigma$ or $\sigma^{\prime}$. We fix an isomorphism $i: \overline{\mathbb{Q}}_{p} \cong \mathbb{C}$, let $\tau_{\mathbb{C}}=\tau \otimes_{\mathcal{O}} \mathbb{C}$ and let $\tau_{\mathbb{C}}^{*}$ be the $\mathbb{C}$ linear dual of $\tau$. Since $U \cap\left(\mathbb{A}_{F}^{f}\right)^{\times}$acts trivially on $\tau$ by assumption, we may consider $\tau_{\mathbb{C}}^{*}$ as a representation of $U\left(\mathbb{A}_{F}^{f}\right)^{\times}$, on which $\left(\mathbb{A}_{F}^{f}\right)^{\times}$acts by $\psi$. Let $U^{\prime}=\prod_{v} U_{v}^{\prime}$ be an open subgroup of $U$ such that $U_{v}^{\prime}=U_{v}$, if $v \nmid 2$ and $U_{v}^{\prime}=\left\{g \in U_{v}: g \equiv 1(\bmod 2)\right\}$ for all $v \mid 2$. Then $U^{\prime}$ acts trivially on $\tau$. Let $C^{\infty}\left(D^{\times} \backslash\left(D \otimes_{F} \mathbb{A}_{F}\right)^{\times} / U^{\prime}\right)$ be the space of smooth $\mathbb{C}$-valued functions on $D^{\times} \backslash\left(D \otimes_{F} \mathbb{A}_{F}\right)^{\times}$which are invariant under $U^{\prime}$. Since $U^{\prime}$ is a normal subgroup of $U, U$ acts on this space by right translations. It follows from [Kisin 2009c, §3.1.14; Taylor 2006, Lemma 1.3] that we have an isomorphism

$$
S_{\tau, \psi}(U, \mathcal{O}) \otimes_{\mathcal{O}} \mathbb{C} \cong \operatorname{Hom}_{U\left(\mathbb{A}_{F}^{f}\right)^{\times}}\left(\tau, C^{\infty}\left(D^{\times} \backslash\left(D \otimes_{F} \mathbb{A}_{F}\right)^{\times} / U^{\prime} D_{\infty}^{\times}\right)\right) .
$$

This isomorphism is equivariant for the Hecke operators at $v \notin S$. The action of $R_{F, S}^{\psi, \square}(\tau)$ on $S_{\tau, \psi}(U, \mathcal{O})_{\mathfrak{m}}$ factors through the action of the Hecke algebra $\mathbb{T}_{\tau, \psi}(U)$. Let $\mathfrak{n}$ be a maximal ideal of $\mathbb{T}(U)_{\tau, \psi}\left[\frac{1}{2}\right]$. The isomorphism above implies that $S_{\tau, \psi}(U, \mathcal{O})_{\mathfrak{n}}$ is nonzero if and only if there is an automorphic form

$$
f^{D} \in C^{\infty}\left(D^{\times} \backslash\left(D \otimes_{F} \mathbb{A}_{F}\right)^{\times} / U^{\prime} D_{\infty}^{\times}\right),
$$

on which the Hecke operators for $v \notin S$ act by the eigenvalues given by the map $\mathbb{T}_{\tau, \psi}(U) \rightarrow \kappa(n) \stackrel{i}{\rightarrow} \mathbb{C}$. Additionally, $\operatorname{Hom}_{U\left(\mathbb{A}_{F}^{f}\right) \times}\left(\tau_{\mathbb{C}}^{*}, \pi\right) \neq 0$, where $\pi=\otimes_{v}^{\prime} \pi_{v}$ is the automorphic representation corresponding to $f^{D}$.

If $S_{\sigma, \psi}(U, \mathcal{O})_{\mathfrak{n}}$ is nonzero then the above implies that $\operatorname{Hom}_{U_{w}}\left(\mathbf{1}, \pi_{w}\right) \neq 0$, which implies that $\pi_{w}$ is an unramified principal series representation, which implies that 
$\operatorname{Hom}_{U_{w}}\left(\tilde{\mathrm{st}}, \pi_{w}\right) \neq 0$. Since $\sigma_{v}=\sigma_{v}^{\prime}$ for all $v \neq w$, we conclude that $S_{\sigma^{\prime}, \psi}(U, \mathcal{O})_{\mathfrak{n}}$ is nonzero.

If $S_{\sigma^{\prime}, \psi}(U, \mathcal{O})_{\mathfrak{n}}$ is nonzero then the same argument shows that $\operatorname{Hom}_{U_{w}}\left(\tilde{\mathrm{st}}, \pi_{w}\right) \neq 0$, which implies that $\pi_{w}$ is either an unramified principal series representation, in which case $\operatorname{Hom}_{U_{w}}\left(\mathbf{1}, \pi_{w}\right) \neq 0$ and thus $S_{\sigma, \psi}(U, \mathcal{O})_{\mathfrak{n}} \neq 0$, or $\pi_{w}$ is a special series. We would like to rule the last case out. By Jacquet-Langlands correspondence to $\pi$ we may associate an automorphic representation $\pi^{\prime}=\otimes_{v}^{\prime} \pi_{v}^{\prime}$ of $\mathrm{GL}_{2}\left(\mathbb{A}_{F}\right)$ such that $\pi_{v}=\pi_{v}^{\prime}$ for all $v$, where $D$ is split. In particular, $\pi_{w}^{\prime}=\pi_{w}$. Let $\rho_{\mathfrak{n}}$ be the representation of $G_{F, S}$ corresponding to the maximal ideal $\mathfrak{n}$ of $R_{F, S}^{\psi}\left[\frac{1}{2}\right]$. By the compatibility of local and global Langlands correspondence, if $\pi_{w}^{\prime}$ is special then $\left.\rho\right|_{G_{F w}}$ is semistable noncrystalline. However, this cannot happen, as explained above.

Corollary 3.21. Assume that $\psi$ is trivial on $U \cap\left(\mathbb{A}_{F}^{f}\right)^{\times}, \sigma_{v}$ is either $\tilde{\mathbf{1}}$ or $\tilde{\mathrm{st}}$ for all $v \mid 2$, and $\left.\bar{\rho}\right|_{G_{v}}$ does not have scalar semisimplification for any $v \mid 2$. If $S_{\sigma, \psi}(U, \mathcal{O})_{\mathfrak{m}} \neq 0$ then the equivalent conditions of Proposition 3.17 hold.

Proof. If $\sigma_{v}=\tilde{\mathbf{1}}$ for all $v \mid 2$ then the assertion is proved in Corollary 3.19. Using this case and Lemma 3.20 we may show that part (d) of Proposition 3.17 is verified for all $\sigma$ as above.

3E. Computing Hilbert-Samuel multiplicity. Let $\sigma=\bigotimes_{v \mid 2} \sigma_{v}$ be a continuous representation of $U$ on a finitely generated $\mathcal{O}$-module $W_{\sigma}$, where the $\sigma_{v}$ are of the form (30) or (31). Let $\psi:\left(\mathbb{A}_{F}^{f}\right)^{\times} / F^{\times} \rightarrow \mathcal{O}^{\times}$be a continuous character such that $U \cap\left(\mathbb{A}_{F}^{f}\right)^{\times}$acts on $W_{\sigma}$ by the character $\psi$. Let $\bar{\sigma}$ and $\bar{\psi}$ be representations obtained by reducing $\sigma$ and $\psi$ modulo $\varpi$. We assume that $U$ satisfies (29), which implies that the subgroups $U_{Q_{n}}$ also satisfy (29). Hence, the functor $\sigma \mapsto S_{\sigma, \psi}\left(U_{Q_{n}}, \mathcal{O}\right)$ is exact. We note that since $R_{F, S}^{\psi, \square}$ is formally smooth over $R_{F, S}^{\psi}$, it is a flat $R_{F, S^{-}}^{\psi}$ module; therefore, the functor $\otimes_{R_{F, S}^{\psi}} R_{F, S}^{\psi, \square}$ is exact, and so is the localization at $\mathfrak{m}_{Q_{n}}$. Hence the functor

$$
\sigma \mapsto M_{n}(\sigma)=R_{F, S_{Q_{n}}}^{\psi, \square} \otimes_{R_{F, S_{Q_{n}}}^{\psi}} S_{\sigma, \psi}\left(U_{Q_{n}}, \mathcal{O}\right)_{\mathfrak{m}_{Q_{n}}}
$$

is exact. Following [Kisin 2009a, §2.2.5] we fix a $U$-invariant filtration on $\bar{\sigma}$ by $k$-subspaces

$$
0=L_{0} \subset L_{1} \subset \cdots \subset L_{s}=W_{\sigma} \otimes_{\mathcal{O}} k
$$

such that, for $i=0,1, \ldots, s-1, \sigma_{i}:=L_{i+1} / L_{i}$ is absolutely irreducible. Since the functor in (45) is exact, this induces a filtration on $M_{n}(\sigma) \otimes_{\mathcal{O}} k$, which we denote by

$$
0=M_{n}^{0}(\sigma) \subset M_{n}^{1}(\sigma) \subset \cdots \subset M_{n}^{s}(\sigma)=M_{n}(\sigma) \otimes_{\mathcal{O}} k
$$


such that, for $i=0,1, \ldots, s-1$, we have

$$
M_{n}^{i+1}(\sigma) / M_{n}^{i}(\sigma) \cong M_{n}\left(\sigma_{i}\right) .
$$

Each representation $\sigma_{i}$ is of the form $\bigotimes_{v \mid 2} \sigma_{i, v}$, where $\sigma_{i, v}$ is either the trivial representation, in which case we let $\tilde{\sigma}_{i, v}=\tilde{\mathbf{1}}$, or st, in which case we let $\tilde{\sigma}_{i, v}:=\tilde{\text { stt. We }}$ let $\tilde{\sigma}_{i}:=\bigotimes_{v \mid 2} \tilde{\sigma}_{i, v}$ and consider it as a representation of $U$ by letting $U_{v}$ for $v$ not above 2 act trivially. We note that, since both $\tilde{\mathbf{1}}$ and $\tilde{\text { st }}$ have trivial central character, $U \cap\left(\mathbb{A}_{F}^{f}\right)^{\times}$acts trivially on $\tilde{\sigma}_{i}$. We choose a continuous character $\xi: F^{\times} \backslash\left(\mathbb{A}_{F}^{f}\right)^{\times} \rightarrow \mathcal{O}^{\times}$such that $\psi \equiv \xi(\bmod \varpi)$ and the restriction of $\xi$ to $U \cap\left(\mathbb{A}_{F}^{f}\right)^{\times}$ is trivial. For example, we could choose $\xi$ to be a Teichmüller lift of $\bar{\psi}$. Let

$$
M_{n}\left(\tilde{\sigma}_{i}\right)=R_{F, S_{Q_{n}}^{\xi, \square}} \otimes_{R_{F, S_{Q_{n}}}^{\xi}} S_{\tilde{\sigma}_{i}, \xi}\left(U_{Q_{n}}, \mathcal{O}\right)_{\mathfrak{m}_{Q_{n}}} .
$$

The exactness of the functor in (45), used with $\tilde{\sigma}_{i}$ and $\xi$ instead of $\sigma$ and $\psi$, and (47) give us an isomorphism

$$
\alpha_{i, n}: M_{n}^{i+1}(\sigma) / M_{n}^{i}(\sigma) \cong M_{n}\left(\sigma_{i}\right) \cong M_{n}\left(\tilde{\sigma}_{i}\right) \otimes_{\mathcal{O}} k .
$$

The isomorphism $\alpha_{i, n}$ is equivariant for the action of the Hecke operators outside $S_{Q_{n}}$, since they act by the same formulas on all the modules. Hence (48) is an isomorphism of $R_{S}^{\square} \llbracket x_{1}, \ldots, x_{g} \rrbracket$-modules. We let $\mathfrak{a}_{i, n}$ be the $R_{F, S_{Q_{n}}}^{\xi, \square}\left(\tilde{\sigma}_{i}\right)$-annihilator of $M_{n}\left(\tilde{\sigma}_{i}\right) \otimes_{\mathcal{O}} k$. Since the action of $R_{S}^{\square} \llbracket x_{1}, \ldots, x_{g} \rrbracket$ on $M_{n}(\sigma)$ and $M_{n}\left(\tilde{\sigma}_{i}\right)$ factors through $R_{F, S_{Q_{n}}}^{\psi, \square}(\sigma)$ and $R_{F, S_{Q_{n}}}^{\xi, \square}\left(\tilde{\sigma}_{i}\right)$, respectively, we obtain a surjection

$$
\varphi_{i, n}: R_{F, S_{Q_{n}}}^{\psi, \square}(\sigma) \rightarrow R_{F, S_{Q_{n}}}^{\xi, \square}\left(\tilde{\sigma}_{i}\right) / \mathfrak{a}_{i, n} .
$$

Proposition 3.22. We may patch in such a way that:

- There is an $R_{\infty}(\sigma)$-module $M_{\infty}(\sigma)$ as in Section $3 C$.

- There is a filtration

$$
0=M_{\infty}^{0}(\sigma) \subset M_{\infty}^{1}(\sigma) \subset \cdots \subset M_{\infty}^{s}(\sigma)=M_{\infty}(\sigma) \otimes_{\mathcal{O}} k
$$

by $R_{\infty}(\sigma)$-submodules.

- For each $1 \leq i \leq s$ there is an $R_{\infty}\left(\tilde{\sigma}_{i}\right)$-module $M_{\infty}\left(\tilde{\sigma}_{i}\right)$ as in Section $3 C$ and a surjection $\varphi_{i}: R_{\infty}(\sigma) \rightarrow R_{\infty}\left(\tilde{\sigma}_{i}\right) / \mathfrak{a}_{i}$, where $\mathfrak{a}_{i}$ is the $R_{\infty}\left(\tilde{\sigma}_{i}\right)$-annihilator of $M_{\infty}\left(\tilde{\sigma}_{i}\right) \otimes_{\mathcal{O}} k$, which allows us to consider $M_{\infty}\left(\tilde{\sigma}_{i}\right) \otimes_{\mathcal{O}} k$ as an $R_{\infty}(\sigma)$-module.

- For each $1 \leq i \leq s$ there is an isomorphism of $R_{\infty}(\sigma)$-modules

$$
\alpha_{i}: M_{\infty}^{i}(\sigma) / M_{\infty}^{i-1}(\sigma) \cong M_{\infty}\left(\tilde{\sigma}_{i}\right) \otimes_{\mathcal{O}} k
$$

Proof. We modify the proof of [Khare and Wintenberger 2009b, Proposition 9.3], which in turn is a modification of the proof of [Kisin 2009c, Proposition 3.3.1]. Let $\Delta(\sigma)_{m}:=\left(D(\sigma)_{m}, L(\sigma)_{m}, D^{\prime}(\sigma)_{m}\right)$ be the patching data of level $m$ as in the proof 
of [Khare and Wintenberger 2009b, Proposition 9.3], where $\sigma$ indicates the fixed weight and inertial type we are working with. In particular, $D(\sigma)_{m}$ and $D^{\prime}(\sigma)_{m}$ are finite $R_{S}^{\psi, \square}(\sigma) \llbracket x_{1}, \ldots, x_{g} \rrbracket$-algebras, where $g=h+j+t-d$, and $L(\sigma)_{m}$ is a module over $D(\sigma)_{m}$ satisfying a number of conditions, listed in the proof of [Khare and Wintenberger 2009b, Proposition 9.3]. Our patching data of level $m$ consists of tuples

$$
\Delta_{m}:=\left(\Delta(\sigma)_{m},\left\{L(\sigma)_{m}^{i}\right\}_{i=0}^{s},\left\{\Delta\left(\tilde{\sigma}_{i}\right)_{m}\right\}_{i=1}^{s},\left\{\varphi_{i, m}\right\}_{i=1}^{s},\left\{\alpha_{i, m}\right\}_{i=1}^{s}\right),
$$

where $\left\{L(\sigma)_{m}^{i}\right\}_{i=0}^{s}$ is a filtration of $L(\sigma)_{m} \otimes_{\mathcal{O}} k$ by $D(\sigma)_{m}$-submodules, $\varphi_{i, m}$ : $D(\sigma)_{m} \rightarrow D\left(\tilde{\sigma}_{i}\right)_{m} / \mathfrak{a}_{i, m}$ is a surjection of $R_{S}^{\square} \llbracket x_{1}, \ldots, x_{g} \rrbracket$-algebras, where $\mathfrak{a}_{i, m}$ is the $D\left(\tilde{\sigma}_{i}\right)_{m}$-annihilator of $L\left(\tilde{\sigma}_{i}\right) \otimes_{\mathcal{O}} k$, and $\alpha_{i, m}$ is an isomorphism of $D(\sigma)_{m}$ modules between $L(\sigma)_{m}^{i} / L(\sigma)_{m}^{i-1}$ and $L\left(\tilde{\sigma}_{i}\right) \otimes_{\mathcal{O}} k$, where the action of $D(\sigma)_{m}$ on this last module is given by $\varphi_{i, m}$.

An isomorphism of patching data between $\Delta_{m}$ and $\Delta_{m}^{\prime}$ is a tuple $\left(\beta,\left\{\beta_{i}\right\}_{i=1}^{s}\right)$, where $\beta: \Delta_{m}(\sigma) \cong \Delta_{m}^{\prime}(\sigma)$ and $\beta_{i}: \Delta_{m}\left(\tilde{\sigma}_{i}\right) \cong \Delta_{m}\left(\tilde{\sigma}_{i}\right)$ are isomorphisms of patching data, in the sense of [Khare and Wintenberger 2009b, Proposition 9.3], which respect the filtration and the maps $\left\{\varphi_{i, m}\right\}_{i=1}^{s},\left\{\alpha_{i, m}\right\}_{i=1}^{s}$. There are only finitely many isomorphism classes of patching data of level $m$, since there are only finitely many isomorphism classes of patching data of level $m$ in the sense of [Khare and Wintenberger 2009b, Proposition 9.3], and a finite $\mathcal{O}$-module can admit only finitely many filtrations and there are only finitely many maps between two finite modules.

We then proceed as in the proof of [Khare and Wintenberger 2009b, Proposition 9.3]. In particular, the integers $a, r_{m}, n_{0}$ and ideals $\mathfrak{c}_{m}$ and $\mathfrak{b}_{n}$ are those defined in [loc. cit.]. For an integer $n \geq n_{0}+1$ and for $m$ with $n \geq m \geq 3$, let $\Delta_{n, m}(\sigma)=$ $\left(D(\sigma)_{n, m}, L(\sigma)_{n, m}, D^{\prime}(\sigma)_{n, m}\right)$ be the patching data of level $m$ as in the proof of [Khare and Wintenberger 2009b, Proposition 9.3]. Then

$$
\begin{aligned}
& D(\sigma)_{n, m}=R_{n+a}(\sigma) /\left(\mathfrak{c}_{m} R_{n+a}(\sigma)+\mathfrak{m}_{R_{n+a}(\sigma)}^{\left(r_{m}\right)}\right), \\
& L(\sigma)_{n, m}=M_{n+a}(\sigma) / \mathfrak{c}_{m} M_{n+a}(\sigma),
\end{aligned}
$$

where $R_{n}(\sigma):=R_{F, S_{Q_{n}}}^{\psi, \square}(\sigma)$. We define $\Delta_{n, m}\left(\tilde{\sigma}_{i}\right)$ analogously with $\tilde{\sigma}_{i}$ instead of $\sigma$ and with $\xi$ instead of $\psi$. We let $\left(L(\sigma)_{n, m}^{i}\right)_{i=1}^{s}$ be the filtration obtained by reducing (46) modulo $\mathfrak{c}_{m}$. Similarly, we let $\left\{\varphi_{i, n, m}\right\}_{i=1}^{s},\left\{\alpha_{i, n, m}\right\}_{i=1}^{s}$ be the maps obtained by reducing (48) and (49) modulo $\mathfrak{c}_{m}$. Then

$$
\left.\Delta_{n, m}:=\left(\Delta(\sigma)_{n, m},\left\{L(\sigma)_{n, m}^{i}\right\}_{i=0}^{s},\left\{\Delta\left(\tilde{\sigma}_{i}\right)_{n, m}\right\}_{i=1}^{s},\left\{\varphi_{i, n, m}\right\}_{i=1}^{s},\left\{\alpha_{i, n, m}\right\}_{i=1}^{s}\right\}\right)
$$

is a patching datum of level $m$ in our sense. Since there are only finitely many isomorphism classes of patching data of level $m$, after replacing the sequence

$$
\left(\left(R_{n+a}(\sigma), M_{n+a}(\sigma)\right),\left\{\left(R_{n+a}\left(\tilde{\sigma}_{i}\right), M_{n+a}\left(\tilde{\sigma}_{i}\right)\right)\right\}_{i=1}^{s}\right)_{n \geq n_{0}+1}
$$


by a subsequence, we may assume that, for each $m \geq n_{0}+4$ and all $n \geq m$, we have $\Delta_{m, n}=\Delta_{m, m}$. The patching data $\Delta_{m, m}$ form a projective system; see [Kisin 2009c, Proposition 3.3.1]. We obtain the desired objects by passing to the limit.

We need to control the image of $R_{\infty}^{\text {inv }}(\sigma)$ under $\varphi_{i}$. Following [Khare and Wintenberger 2009b] we let $\mathrm{CNL}_{\mathcal{O}}$ be the category of complete local noetherian $\mathcal{O}$-algebras with a fixed isomorphism of the residue field with $k$, and whose maps are local $\mathcal{O}$-algebra homomorphisms. If $A \in \mathrm{CNL}_{\mathcal{O}}$ then we let $\mathrm{Sp}_{A}: \mathrm{CNL}_{\mathcal{O}} \rightarrow$ Sets be the functor $\operatorname{Sp}_{A}(B)=\operatorname{Hom}_{\mathrm{CNL}_{\mathcal{O}}}(A, B)$. Let $G$ be a finite abelian group. We let $G^{*}$ be the group scheme defined over $\mathcal{O}$ such that, for every $\mathcal{O}$-algebra $A$, $G^{*}(A)=\operatorname{Hom}_{\text {Groups }}\left(G, A^{\times}\right)$. Assume that we are given a free $G^{*}$ action on $\operatorname{Sp}_{A}$. This means that, for all $B \in \mathrm{CNL}_{\mathcal{O}}, G^{*}(B)$ acts on $\operatorname{Sp}_{A}(B)$ without fixed points. By Proposition 2.6(1) in [Khare and Wintenberger 2009b] the quotient $G^{*} \backslash \mathrm{Sp}_{A}$ exists in $\mathrm{CNL}_{\mathcal{O}}$ and is represented by $\left(A^{\mathrm{inv}}, \mathfrak{m}_{A}^{\mathrm{inv}}\right) \in \mathrm{CNL}_{\mathcal{O}}$. Moreover, $\mathrm{Sp}_{A}$ is a $G^{*}$-torsor over $\mathrm{Sp}_{A^{\text {inv }}}$.

Lemma 3.23. Let $\left(A, \mathfrak{m}_{A}\right)$ and $\left(B, \mathfrak{m}_{B}\right)$ be in $\mathrm{CNL}_{\mathcal{O}}$. Assume that $G^{*}$ acts freely on $\mathrm{Sp}_{A}$ and $\mathrm{Sp}_{B}$ and we are given $a G^{*}$-equivariant closed immersion $\mathrm{Sp}_{B} \hookrightarrow \mathrm{Sp}_{A}$. Then the map induces a closed immersion $\mathrm{Sp}_{B^{\text {inv }}} \hookrightarrow \mathrm{Sp}_{A^{\text {inv }}}$.

Proof. Since $G^{*}$ acts trivially on $\mathrm{Sp}_{A^{\text {inv }}}$, by the universal property of the quotient, the map $\mathrm{Sp}_{B} \rightarrow \mathrm{Sp}_{A} \rightarrow \mathrm{Sp}_{A^{\text {inv }}}$ factors through $\mathrm{Sp}_{B^{\text {inv }}} \rightarrow \mathrm{Sp}_{A^{\text {inv }}}$. Hence, we obtain the following commutative diagram in $\mathrm{CNL}_{\mathcal{O}}$ :

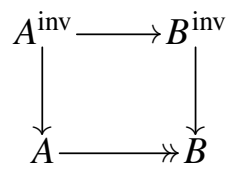

Since $\mathrm{Sp}_{A}$ is a $G^{*}$-torsor over $\mathrm{Sp}_{A^{\text {inv }}}$, it follows from [SGA $3_{\text {II }} 1970$, Exposé VIII, Proposition 4.1] that $A$ is a free $A^{\text {inv }}$-module of rank $|G|$. Similarly, $B$ is a free $B^{\text {inv }}$ module of rank $|G|$. It follows from the commutative diagram that the surjection $A \rightarrow B$ induces a surjection $A / \mathfrak{m}_{A}^{\text {inv }} A \rightarrow B / \mathfrak{m}_{B}^{\text {inv }} B$. Since both $k$-vector spaces have dimension $|G|$, the map is an isomorphism and this implies that the image of $\mathfrak{m}_{A}^{\text {inv }}$ is equal to $\mathfrak{m}_{B}^{\text {inv }}$. Hence, the top horizontal arrow in the diagram is surjective.

Let $\mathrm{CNL}_{\mathcal{O}}^{[m]}$ be the full subcategory of $\mathrm{CNL}_{\mathcal{O}}$ consisting of objects $\left(A, \mathfrak{m}_{A}\right)$ such that $\mathfrak{m}_{A}^{m}=0$. We have a truncation functor $\mathrm{CNL}_{\mathcal{O}} \rightarrow \mathrm{CNL}_{\mathcal{O}}^{[m]}, A \mapsto A^{[m]}:=A / \mathfrak{m}_{A}^{m}$. If $A$ represents the functor $X$, we denote by $X^{[m]}$ the functor represented by $A^{[m]}$. For group chunk actions, we refer the reader to [Khare and Wintenberger 2009b, §2.6].

Lemma 3.24. Let $\left(A, \mathfrak{m}_{A}\right)$ and $\left(B, \mathfrak{m}_{B}\right)$ be in $\mathrm{CNL}_{\mathcal{O}}$. Assume that $G^{*}$ acts freely on $X:=\mathrm{Sp}_{A}$ and $Y:=\mathrm{Sp}_{B}$ and we are given an isomorphism $X^{[m]} \cong Y^{[m]}$ compatible with the group chunk $\left(G^{*}\right)^{[m]}$-action. If $m$ is large enough then the image of $\mathfrak{m}_{A}^{\mathrm{inv}} A$ in $A / \mathfrak{m}_{A}^{m}=B / \mathfrak{m}_{B}^{m}$ is equal to the image of $\mathfrak{m}_{B}^{\text {inv }} B$. 
Proof. Let $X^{\text {inv }}$ and $Y^{\text {inv }}$ denote the quotients of $X$ and $Y$ by $G^{*}$. Then we have isomorphisms

$$
G^{*} \times X \cong X \times_{X^{\text {inv }}} X, \quad G^{*} \times Y \cong Y \times_{Y^{\text {inv }}} Y,
$$

where the map is given by $(g, x) \mapsto(x, g x)$. We define $Z:=X^{[m]}=Y^{[m]}$ and $C:=A / \mathfrak{m}_{A}^{m}=B / \mathfrak{m}_{B}^{m}$. The restriction of the above isomorphism to $\mathrm{CNL}_{\mathcal{O}}^{[m]}$ gives us isomorphisms

$$
\left(G^{*} \times Z\right)^{[m]} \cong\left(Z \times_{X^{\text {inv }}} Z\right)^{[m]}, \quad\left(G^{*} \times Z\right)^{[m]} \cong\left(Z \times_{Y^{\text {inv }}} Z\right)^{[m]} .
$$

Thus we have an isomorphism

$$
\left(Z \times_{X^{\text {inv }}} Z\right)^{[m]} \cong\left(Z \times_{Y^{\text {inv }}} Z\right)^{[m]},
$$

where the map is given by $\left(z_{1}, z_{2}\right) \mapsto\left(z_{1}, z_{2}\right)$. On rings this isomorphism reads $\left(C \otimes_{A^{\text {inv }}} C\right)^{[m]} \cong\left(C \otimes_{B^{\text {inv }}} C\right)^{[m]}, c_{1} \otimes c_{2} \mapsto c_{1} \otimes c_{2}$.

Both $A / \mathfrak{m}_{A}^{\text {inv }} A$ and $B / \mathfrak{m}_{B}^{\text {inv }} B$ are $k$-vector spaces of dimension $|G|$. In particular, if $m>|G|$ then $\mathfrak{m}_{A}^{m} \subset \mathfrak{m}_{A}^{\text {inv }} A$ and $\mathfrak{m}_{B}^{m} \subset \mathfrak{m}_{B}^{\text {inv }} B$. So we obtain a map $C \rightarrow A / \mathfrak{m}_{A}^{\text {inv }} A$. If $m>2|G|$ then by base changing along this map, we obtain an isomorphism

$$
A / \mathfrak{m}_{A}^{\text {inv }} A \otimes_{k} A / \mathfrak{m}_{A}^{\text {inv }} A \cong A / \mathfrak{m}_{A}^{\text {inv }} A \otimes_{B^{\text {inv }}} A / \mathfrak{m}_{A}^{\text {inv }} A .
$$

If the image of $B^{\text {inv }}$ in $A / \mathfrak{m}_{A}^{\text {inv }} A$ is not equal to $k$ then, for some $b \in B^{\text {inv }}, 1 \otimes b$ and $b \otimes 1$ will be linearly independent over $k$ in the left-hand side of the above isomorphism and linearly dependent in the right-hand side. This implies that the image of $B^{\text {inv }}$ in $A / \mathfrak{m}_{A}^{\text {inv }} A$ is equal to $k$. Thus $\mathfrak{m}_{B}^{\text {inv }} C \subset \mathfrak{m}_{A}^{\text {inv }} C$ and by symmetry we obtain the other inclusion.

Let $G_{n}$ be the Galois group of the maximal abelian extension of $F$, of degree a power of 2, which is unramified outside $Q_{n}$ and split at primes in $S$. Let $G_{n, 2}=$ $G_{n} / 2 G_{n}$. It follows from [Khare and Wintenberger 2009b, Lemma 5.1(f)] that $G_{n, 2} \cong(\mathbb{Z} / 2 \mathbb{Z})^{t}$. Let $G_{n, 2}^{*}$ be the group scheme defined over $\mathcal{O}$ such that, for every $\mathcal{O}$-algebra $A, G_{n, 2}^{*}(A)=\operatorname{Hom}_{\text {Groups }}\left(G_{n, 2}, A^{\times}\right)$. For a local artinian augmented $\mathcal{O}$-algebra $A$ and $\chi \in G_{n, 2}^{*}(A)$, if $\rho_{A}$ is a $G_{F, S_{Q_{n}}}$-representation lifting $\bar{\rho}$ to $A$ then so is $\rho_{A} \otimes \chi$. Moreover, since $\chi^{2}$ is trivial, $\rho_{A}$ and $\rho_{A} \otimes \chi$ have the same determinant. This induces an action of $G_{n, 2}^{*}$ on

$$
\operatorname{Spf} R_{F, S_{Q_{n}}}^{\square}, \quad \operatorname{Spf} R_{F, S_{Q_{n}}}^{\psi, \square}(\sigma), \quad \text { and } \quad \operatorname{Spf} R_{F, S_{Q_{n}}}^{\xi, \square}\left(\tilde{\sigma}_{i}\right) .
$$

It follows from [Khare and Wintenberger 2009b, Lemma 5.1] that this action is free. Proposition 2.6 of [Khare and Wintenberger 2009b] implies that the quotient by $G_{n, 2}^{*}$ is represented by a complete local noetherian $\mathcal{O}$-algebra, which we will denote by $\left(R_{F, S_{Q_{n}}}^{\square \text {,inv }}, \mathfrak{m}_{n}^{\text {inv }}\right),\left(R_{F, S_{Q_{n}}}^{\psi, \square, \text { inv }}(\sigma), \mathfrak{m}_{n, \sigma}^{\text {inv }}\right)$ and $\left(R_{F, S_{Q_{n}}}^{\xi, \square \text {,inv }}\left(\tilde{\sigma}_{i}\right), \mathfrak{m}_{n, \tilde{\sigma}_{i}}^{\text {inv }}\right)$, respectively. 
Lemma 3.25. The map

$$
\operatorname{Spf} R_{F, S_{Q_{n}}}^{\xi, \square}\left(\tilde{\sigma}_{i}\right) / \mathfrak{a}_{i, n} \rightarrow \operatorname{Spf} R_{F, S_{Q_{n}}}^{\psi, \square}(\sigma)
$$

induced by (49) is $G_{n, 2}^{*}$-equivariant. Moreover,

$$
\varphi_{i, n}\left(\mathfrak{m}_{n, \sigma}^{\mathrm{inv}} R_{F, S_{Q_{n}}}^{\psi, \square}(\sigma)\right)=\mathfrak{m}_{n, \tilde{\sigma}_{i}}^{\mathrm{inv}} R_{F, S_{Q_{n}}}^{\xi, \square}\left(\tilde{\sigma}_{i}\right) / \mathfrak{a}_{i, n} .
$$

Proof. The first part follows from [Khare and Wintenberger 2009b, Lemma 9.1]; see the paragraph after the proof of Proposition 7.6 and the third paragraph of the proof of Lemma 9.6 of the same paper.

Let

$$
q_{\sigma}: R_{F, S_{Q_{n}}}^{\square} \rightarrow R_{F, S_{Q_{n}}}^{\psi, \square}(\sigma) \quad \text { and } \quad q_{\tilde{\sigma}_{i}}: R_{F, S_{Q_{n}}}^{\square} \rightarrow R_{F, S_{Q_{n}}}^{\xi, \square}\left(\tilde{\sigma}_{i}\right)
$$

denote the natural surjections. Since $\varphi_{i, n} \circ q_{\sigma}=q_{\tilde{\sigma}_{i}}\left(\bmod \mathfrak{a}_{n, i}\right)$, it is enough to show that $q_{\sigma}\left(\mathfrak{m}_{n}^{\text {inv }} R_{F, S_{Q_{n}}}^{\square}\right)=\mathfrak{m}_{n, \sigma}^{\text {inv }} R_{F, S_{Q_{n}}}^{\psi, \square}(\sigma)$ for all $\sigma$ and $\psi$ as above. This follows from Lemma 3.23.

Let $\mathfrak{m}_{\sigma}^{\text {inv }}$ and $\mathfrak{m}_{\tilde{\sigma}_{i}}^{\text {inv }}$ be the maximal ideals of $R_{\infty}^{\text {inv }}(\sigma)$ and $R_{\infty}^{\text {inv }}\left(\tilde{\sigma}_{i}\right)$, respectively.

Proposition 3.26. The surjection $\varphi_{i}: R_{\infty}(\sigma) \rightarrow R_{\infty}\left(\tilde{\sigma}_{i}\right) / \mathfrak{a}_{i}$ maps $\mathfrak{m}_{\sigma}^{\text {inv }} R_{\infty}(\sigma)$ onto the image of $\mathfrak{m}_{\tilde{\sigma}_{i}}^{\text {inv }} R_{\infty}\left(\tilde{\sigma}_{i}\right)$. In particular,

$$
e\left(M_{\infty}^{i}(\sigma) / M_{\infty}^{i-1}(\sigma), R_{\infty}^{\mathrm{inv}}(\sigma) / \varpi\right)=e\left(M_{\infty}\left(\tilde{\sigma}_{i}\right) \otimes_{\mathcal{O}} k, R_{\infty}^{\mathrm{inv}}\left(\tilde{\sigma}_{i}\right) / \varpi\right) .
$$

Proof. If $(A, \mathfrak{m})$ is a complete local noetherian algebra then by $A^{[r]}$ we denote the ring $A / \mathfrak{m}^{r}$. We will use the same notation as in the proof of the previous proposition. It is shown in the course of the proof of part (I) of [Khare and Wintenberger 2009b, Proposition 9.3] that

$$
R_{\infty}(\sigma) \cong{\underset{m}{\lim }}_{m, m}^{\prime \prime}(\sigma)
$$

where $D_{m, n}^{\prime \prime}(\sigma)=R_{n+a}(\sigma)^{\left[r_{m}^{\prime}\right]}$. Moreover, it is shown that the map is $\left(\widehat{\mathbb{G}}_{m}[2]\right)^{t}$ equivariant by fixing an identification of $G_{n+a, 2}$ with $(\mathbb{Z} / 2 \mathbb{Z})^{t}$.

For each fixed $r \geq 0$ we have

$$
R_{\infty}(\sigma)^{[r]} \cong \underset{m}{\lim } D_{m, m}^{\prime \prime}(\sigma)^{[r]} .
$$

Hence, by choosing $m$ large enough we may assume that $R_{\infty}(\sigma)^{[r]}=D_{m, m}^{\prime \prime}(\sigma)^{[r]}$ with $r \leq r_{m}^{\prime}$. Since $\left(\widehat{\mathbb{G}}_{m}[2]\right)^{t}$-action on $\operatorname{Sp}_{R_{\infty}(\sigma)}$ and on $\operatorname{Sp}_{R_{n+a}(\sigma)}$ is free by [Khare and Wintenberger 2009b, Lemmas 5.1 and 9.4], we are in the situation of Lemma 3.24. Hence the image of $\mathfrak{m}_{\sigma}^{\text {inv }} R_{\infty}(\sigma)$ in $D_{m, m}^{\prime \prime}(\sigma)^{[r]}$ is equal to the image of $\mathfrak{m}_{m+a, \sigma}^{\text {inv }} R_{m+a}(\sigma)$. It follows from Lemma 3.25 that the composition

$$
R_{\infty}(\sigma) \longrightarrow R_{m+a}(\sigma)^{[r]} \stackrel{\varphi_{i, m}}{\longrightarrow}\left(R_{m+a}\left(\tilde{\sigma}_{i}\right) / \mathfrak{a}_{i, m}\right)^{[r]}
$$


maps $\mathfrak{m}_{\sigma}^{\text {inv }} R_{\infty}(\sigma)$ onto the image of $\mathfrak{m}_{\tilde{\sigma}_{i}}^{\text {inv }} R_{\infty}\left(\tilde{\sigma}_{i}\right)$. The action of $R_{m+a}\left(\tilde{\sigma}_{i}\right)$ on $L_{m, m}\left(\tilde{\sigma}_{i}\right)$ factors through $R_{m+a}\left(\tilde{\sigma}_{i}\right)^{\left[r_{m}^{\prime}\right]}$. Since by construction

$$
\varphi_{i}={\underset{m}{\lim }}_{\varphi_{i, m}}, \quad R_{\infty}\left(\tilde{\sigma}_{i}\right)={\underset{m}{\lim }}_{R_{m+a}}\left(\tilde{\sigma}_{i}\right)^{\left[r_{m}^{\prime}\right]}, \quad M_{\infty}\left(\tilde{\sigma}_{i}\right)={\underset{m}{\lim }}_{L_{m, m}}\left(\tilde{\sigma}_{i}\right),
$$

we deduce that $\varphi_{i}$ maps $\mathfrak{m}_{\sigma}^{\text {inv }} R_{\infty}(\sigma)$ onto the image of $\mathfrak{m}_{\tilde{\sigma}_{i}}^{\text {inv }} R_{\infty}\left(\tilde{\sigma}_{i}\right)$.

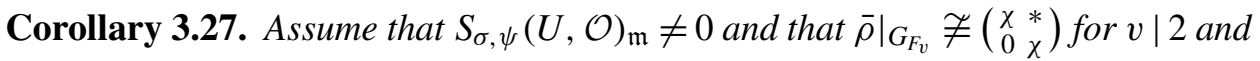
any character $\chi: G_{F_{v}} \rightarrow k^{\times}$. Then the equivalent conditions of Proposition 3.17 hold, and any $\rho: G_{F, S} \rightarrow \mathrm{GL}_{2}(\mathcal{O})$ corresponding to a maximal ideal of $R_{F, S}^{\psi}(\sigma)\left[\frac{1}{2}\right]$ is modular.

Proof. We will verify that part (b) of Proposition 3.17 holds. We first note that, since $S_{\sigma, \psi}(U, \mathcal{O})_{\mathfrak{m}} \neq 0$ and $U$ satisfies (29), there is an $i$ such that $S_{\tilde{\sigma}_{i}, \xi}(U, k)_{\mathfrak{m}} \neq 0$. This implies that $S_{\tilde{\sigma}_{i}, \xi}(U, \mathcal{O})_{\mathfrak{m}} \neq 0$, and it follows from Lemma 3.20 that $S_{\tilde{\sigma}_{i}, \xi}(U, \mathcal{O})_{\mathfrak{m}} \neq 0$ for all $1 \leq i \leq s$ and $S_{\tilde{\mathbf{1}}, \xi}(U, \mathcal{O})_{\mathfrak{m}} \neq 0$. In particular, the rings $R_{S}^{\xi, \square}\left(\tilde{\sigma}_{i}\right)$ are nonzero and equal to $R_{S}^{\xi, \square}(\tilde{\mathbf{1}})$. Corollary 3.21 implies that for all $1 \leq i \leq s$ the equality

$$
2^{t} r e\left(R_{S}^{\xi, \square}\left(\tilde{\sigma}_{i}\right) / \varpi\right)=e\left(M_{\infty}\left(\tilde{\sigma}_{i}\right) / \varpi, R_{\infty}^{\mathrm{inv}}\left(\tilde{\sigma}_{i}\right) / \varpi\right)
$$

holds. Since the Hilbert-Samuel multiplicity is additive in short exact sequences, we have

$$
e\left(M_{\infty}(\sigma) / \varpi, R_{\infty}^{\mathrm{inv}}(\sigma) / \varpi\right)=\sum_{i=1}^{s} e\left(M_{\infty}^{i}(\sigma) / M_{\infty}^{i-1}(\sigma), R_{\infty}^{\mathrm{inv}}(\sigma) / \varpi\right) .
$$

Proposition 3.26 implies that for all $1 \leq i \leq s$ we have

$$
e\left(M_{\infty}^{i}(\sigma) / M_{\infty}^{i-1}(\sigma), R_{\infty}^{\mathrm{inv}}(\sigma) / \varpi\right)=e\left(M_{\infty}\left(\tilde{\sigma}_{i}\right) / \varpi, R_{\infty}^{\mathrm{inv}}\left(\tilde{\sigma}_{i}\right) / \varpi\right) .
$$

Thus

$$
e\left(M_{\infty}(\sigma) / \varpi, R_{\infty}^{\mathrm{inv}}(\sigma) / \varpi\right)=2^{t} r \sum_{i=1}^{s} e\left(R_{S}^{\xi, \square}\left(\tilde{\sigma}_{i}\right) / \varpi\right) .
$$

Thus to verify part (b) of Proposition 3.17 it is enough to show that

$$
e\left(R_{S}^{\psi, \square}(\sigma) / \varpi\right) \leq \sum_{i=1}^{s} e\left(R_{S}^{\xi, \square}\left(\tilde{\sigma}_{i}\right) / \varpi\right) .
$$

If $A$ and $B$ are complete local $\kappa$-algebras with residue field $\kappa$ then it is shown in [Kisin 2009a, Proposition 1.3.8] that $e\left(A \widehat{\otimes}_{\kappa} B\right)=e(A) e(B)$. Since $\psi$ is congruent to $\xi$ modulo $\varpi$, inequality (55) reduces to the following inequality on HilbertSamuel multiplicities of potentially semistable rings at all $v \mid 2$ :

$$
e\left(R_{v}^{\psi, \square}\left(\sigma_{v}\right) / \varpi\right) \leq \sum_{i=1}^{s_{v}} e\left(R_{v}^{\xi, \square}\left(\tilde{\sigma}_{v, i}\right) / \varpi\right) .
$$


Here the $\sigma_{v, i}$ are irreducible $k$-representation of $\mathrm{GL}_{2}\left(\mathbb{F}_{2}\right)$ which appear as graded pieces of a $\mathrm{GL}_{2}\left(\mathbb{Z}_{2}\right)$-invariant filtration on $\sigma_{v} \otimes_{\mathcal{O}} k$. Inequality (56) is proved in the local part of the paper; see Remark 2.39.

3F. Modularity lifting. Let $F$ be a totally real field in which 2 splits completely.

Definition 3.28. An allowable base change is a totally real solvable extension $F^{\prime}$ of $F$ such that 2 splits completely in $F^{\prime}$.

Lemma 3.29. Assume that $[F: \mathbb{Q}]$ is even. Let $\bar{\rho}: G_{F} \rightarrow \mathrm{GL}_{2}(k)$ be a continuous absolutely irreducible representation. If there is a Hilbert eigenform $f$ such that $\bar{\rho} \cong \bar{\rho}_{f}$ then there is a Hilbert eigenform $g$ of parallel weight 2 such that $\bar{\rho} \cong \bar{\rho}_{g}$ and at $v \mid 2$ the corresponding representation $\pi_{v}$ of $\mathrm{GL}_{2}\left(F_{v}\right)$ is either an unramified principal series or a twist of Steinberg representation by an unramified character. Moreover, if $\left.\bar{\rho}\right|_{G_{F_{v}}} ¥\left(\begin{array}{ll}\chi & * \\ 0 & \chi\end{array}\right)$ for all $v \mid 2$ and any character $\chi: G_{F_{v}} \rightarrow k^{\times}$then we may assume that $\pi_{v}$ is an unramified principal series representation for all $v \mid 2$.

Proof. Let $D$ be the totally definite quaternion algebra with center $F$ split at all the finite places. Let $f^{D} \in S_{\tau, \psi}(U, \mathcal{O})$ be the eigenform on $D$ associated to $f$ by the Jacquet-Langlands correspondence, where $U=\prod_{v} U_{v}$ is a compact open subgroup of $\left(D \otimes_{F} \mathbb{A}_{F}^{f}\right)^{\times}$such that $U_{v}=\mathrm{GL}_{2}\left(\mathcal{O}_{F_{v}}\right)$ for all $v \mid 2$, and $U$ is sufficiently small, so that (29) holds, and $\tau=\bigotimes_{v \mid 2} \tau_{v}$ is a locally algebraic representation of $U$. Let $\mathfrak{m}$ be the maximal ideal of the Hecke algebra $\mathbb{T}_{S, \mathcal{O}}^{\text {univ }}$ corresponding to $\bar{\rho}$. Then $f^{D} \in S_{\tau, \psi}(U, \mathcal{O})_{\mathfrak{m}}$, and hence $S_{\tau, \psi}(U, \mathcal{O})_{\mathfrak{m}}$ is nonzero.

Let $\bar{\tau}$ denote the reduction of a $U$-invariant lattice in $\tau$, and let $\bar{\psi}$ denote $\psi$ modulo $\varpi$. Since $U$ satisfies (29) the functor $\sigma \mapsto S_{\sigma, \psi}(U, \mathcal{O})$ is exact. The localization functor is also exact. Hence there is an irreducible subquotient $\sigma$ of $\bar{\tau}$ such that $S_{\sigma, \bar{\psi}}(U, k)_{\mathfrak{m}}$ is nonzero. Such a $\sigma$ is of the form $\bigotimes_{v \mid 2} \sigma_{v}$, where $\sigma_{v}$ is a representation of $\mathrm{GL}_{2}\left(\mathbb{F}_{2}\right)$. Thus $\sigma_{v}$ is either trivial, in which case we let

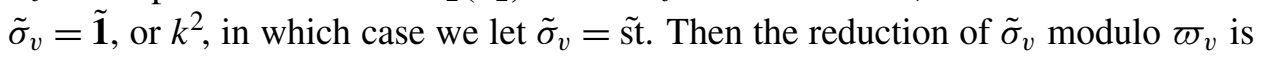
isomorphic to $\sigma_{v}$ and $F_{v}^{\times} \cap U_{v}$ acts trivially on $\tilde{\sigma}_{v}$. Let $\tilde{\sigma}:=\bigotimes_{v \mid 2} \tilde{\sigma}_{v}$. Choose a lift $\xi:\left(\mathbb{A}_{F}^{f}\right)^{\times} / F^{\times} \rightarrow \mathcal{O}^{\times}$of $\bar{\psi}$, which is trivial on $U \cap\left(\mathbb{A}_{F}^{f}\right)^{\times}$. The exactness of the functor $\sigma \mapsto S_{\sigma, \xi}(U, \mathcal{O})$ implies that $S_{\tilde{\sigma}, \xi}(U, \mathcal{O})_{\mathfrak{m}}$ is nonzero, since its reduction modulo $\varpi$ is equal to $S_{\sigma, \xi}(U, k)_{\mathfrak{m}}$. We may take any eigenform $g^{D} \in S_{\tilde{\sigma}, \tilde{\psi}}(U, \mathcal{O})_{\mathfrak{m}}$ and then using Jacquet-Langlands transfer it to a Hilbert modular form, which will have the prescribed properties. The last part follows from Lemma 3.20.

Theorem 3.30. Let $F$ be a totally real field where 2 is totally split, and let

$$
\rho: G_{F, S} \rightarrow \mathrm{GL}_{2}(\mathcal{O})
$$

be a continuous representation. Suppose: 
(i) $\bar{\rho}: G_{F, S} \stackrel{\rho}{\rightarrow} \mathrm{GL}_{2}(\mathcal{O}) \rightarrow \mathrm{GL}_{2}(k)$ is modular with nonsolvable image.

(ii) If $v \mid 2$ then $\left.\rho\right|_{G_{F_{v}}}$ is potentially semistable with distinct Hodge-Tate weights.

(iii) $\operatorname{det} \rho$ is totally odd.

(iv) If $v \mid 2$ then $\left.\bar{\rho}\right|_{G_{F_{v}}} \not\left(\begin{array}{ll}\chi & * \\ 0 & \chi\end{array}\right)$, for any character $\chi: G_{F_{v}} \rightarrow k^{\times}$.

Then $\rho$ is modular.

Proof. Let $\psi=\chi_{\text {cyc }}^{-1} \operatorname{det} \rho$, where $\chi_{\text {cyc }}$ is the 2 -adic cyclotomic character. By solvable base change it is enough to prove the assertion for the restriction of $\rho$ to $G_{F^{\prime}}$, where $F^{\prime}$ is a totally real solvable extension of $F$. Using Lemma 2.2 of [Taylor 2003] we may find an allowable base change $F^{\prime}$ of $F$ such that $\left[F^{\prime}: \mathbb{Q}\right]$ is even and $\left.\bar{\rho}\right|_{G_{F^{\prime}}}$ is unramified outside places above 2 . We may further assume that if $\rho$ is ramified at $v \nmid 2$ then the image of inertia is unipotent. Let $\Sigma$ be the set of places outside 2 where $\rho$ is ramified. If $v \in \Sigma$ then

$$
\left.\rho\right|_{G_{F_{v}^{\prime}}} \cong\left(\begin{array}{cc}
\gamma_{v} \chi_{\mathrm{cyc}} & * \\
0 & \gamma_{v}
\end{array}\right)
$$

where $\gamma_{v}$ is an unramified character such that $\gamma_{v}^{2}=\left.\psi\right|_{G_{F_{v}^{\prime}}}$.

Since $\bar{\rho}$ is assumed to be modular, Lemma 3.29 implies that $\bar{\rho} \cong \bar{\rho}_{f}$, where $f$ is a Hilbert eigenform of parallel weight 2 , and an unramified principal series at $v \mid$ 2. Using Lemma 3.5.3 of [Kisin 2009c] (see also Theorem 8.4 of [Khare and Wintenberger 2009b]) there is an admissible base change $F^{\prime \prime} / F^{\prime}$ such that $\left.\rho\right|_{G_{F^{\prime \prime}}}$ is ramified at an even number of places outside 2 . We still denote this set by $\Sigma$, and there is a Hilbert eigenform $g$ over $F^{\prime \prime}$ such that $\left.\bar{\rho}\right|_{G_{F^{\prime \prime}}} \cong \bar{\rho}_{g}$, and such that $g$ has parallel weight 2 , is special of conductor 1 at $v \in \Sigma$, and is unramified otherwise.

Let $D$ be the quaternion algebra with center $F^{\prime \prime}$ ramified exactly at all infinite places and all $v \in \Sigma$. Choose a place $v_{1}$ of $F^{\prime \prime}$ as in Lemma 3.2 and such that $\bar{\rho}$ is unramified at $v_{1}$ and $\bar{\rho}\left(\right.$ Frob $\left._{v_{1}}\right)$ has distinct eigenvalues. Let $S$ be the union of infinite places, $\Sigma$, places above 2 and $v_{1}$. Let $U=\prod_{v} U_{v}$ be an open subgroup of $\left(D \otimes_{F^{\prime \prime}} \mathbb{A}_{F^{\prime \prime}}^{f}\right)^{\times}$such that $U_{v}=\mathcal{O}_{D_{v}}^{\times}$if $v \neq v_{1}$ and $U_{v_{1}}$ is unipotent upper triangular modulo $\varpi_{v_{1}}$. We note that Lemma 3.2 implies that $U$ satisfies (29). Let $\mathfrak{m}$ be the maximal ideal in the Hecke algebra $\mathbb{T}_{S, \mathcal{O}}^{\text {univ }}$ corresponding to $\bar{\rho}$.

Let $g^{D}$ be the eigenform on $D$ corresponding to $g$ via the Jacquet-Langlands correspondence. Then $g^{D} \in S_{\sigma, \psi^{\prime}}(U, \mathcal{O})_{\mathfrak{m}}$, where $\sigma$ is the trivial representation of $U$ and $\psi^{\prime}:\left(\mathbb{A}_{F^{\prime \prime}}^{f}\right)^{\times} \rightarrow \mathcal{O}^{\times}$is a suitable character congruent to $\psi$ modulo $\varpi$. In particular, $S_{\sigma, \psi^{\prime}}(U, \mathcal{O})_{\mathfrak{m}} \neq 0$. It follows from Lemma 3.20 that $S_{\sigma, \psi^{\prime}}(U, \mathcal{O})_{\mathfrak{m}} \neq 0$ for all $\sigma=\bigotimes_{v \mid 2} \sigma_{v}$, where $\sigma_{v}$ is either $\tilde{\mathbf{1}}$ or st. Since $U$ satisfies (29), we deduce that $S_{\sigma, \psi}(U, k)_{\mathfrak{m}} \neq 0$ for any irreducible smooth $k$-representation $\sigma$ of $\prod_{v \mid 2} \mathrm{GL}_{2}\left(\mathbb{Z}_{2}\right)$. Since $U$ satisfies (29), we deduce via Lemma 3.1 .4 of [Kisin 2009c] that $S_{\sigma, \psi}(U, \mathcal{O})_{\mathfrak{m}} \neq 0$ for any continuous finite-dimensional representation $\sigma$ of $\prod_{v \mid 2} \mathrm{GL}_{2}\left(\mathbb{Z}_{2}\right)$ on which $U \cap\left(\mathbb{A}_{F^{\prime \prime}}^{f}\right)^{\times}$acts by $\psi$. 
For $v \mid 2$ suppose that $\left.\rho\right|_{G_{F_{v}^{\prime \prime}}}$ has Hodge-Tate weights $\boldsymbol{w}_{v}=\left(a_{v}, b_{v}\right)$ with $b_{v}>a_{v}$ and inertial type $\tau_{v}$. Let $\sigma_{v}$ be defined by (30) and let $\sigma=\bigotimes_{v \mid 2} \sigma_{v}$. The above implies that $S_{\sigma, \psi}(U, \mathcal{O})_{\mathfrak{m}} \neq 0$ and, since $\left.\rho\right|_{G_{F^{\prime \prime}}}$ defines a maximal ideal of $R_{F^{\prime \prime}, S}^{\psi}\left[\frac{1}{2}\right]$, the assertion follows from Corollary 3.27.

\section{Acknowledgements}

The local part was written at about the same time as [Colmez et al. 2014], and it would not have happened if not for the competitive nature of the correspondence I had with Gabriel Dospinescu at the time. I thank Gaëtan Chenevier for the correspondence on 2-dimensional 2-adic determinants. The global part owes a great deal to the work of Khare and Wintenberger [2009b] and Kisin [2009a; 2009b; 2009c] as will be obvious to the reader. I thank Mark Kisin and JeanPierre Wintenberger for answering my questions about their work. I would like to especially thank Toby Gee for his explanations of the Taylor-Wiles-Kisin patching method and for pointing out the right places in the literature to me. I thank Lennart Gehrmann, Jochen Heinloth and Shu Sasaki for a number of stimulating discussions. I thank Gabriel Dospinescu, Matthew Emerton, Toby Gee and Jack Thorne for their comments on the earlier draft. I thank Patrick Allen for pointing out an error in the earlier draft, and for subsequent correspondence, which led to a fix. I thank the referees for their careful reading of the paper.

\section{References}

[Barthel and Livné 1994] L. Barthel and R. Livné, "Irreducible modular representations of GL 2 of a local field”, Duke Math. J. 75:2 (1994), 261-292. MR 1290194 Zbl 0826.22019

[Berger and Breuil 2010] L. Berger and C. Breuil, "Sur quelques représentations potentiellement cristallines de $\mathrm{GL}_{2}\left(\mathbb{Q}_{p}\right)$ ", pp. 155-211 in p-adic representations of p-adic groups, II: Representations of $\mathrm{GL}_{2}\left(\mathbb{Q}_{p}\right)$ and $(\phi, \Gamma)$-modules, edited by L. Berger et al., Astérisque 330, Société Mathématique de France, Paris, 2010. MR 2642406 Zbl 1243.11063

[Blasius 2006] D. Blasius, "Hilbert modular forms and the Ramanujan conjecture", pp. 35-56 in Noncommutative geometry and number theory, edited by C. Consani and M. Marcolli, Aspects Math. E37, Vieweg, Wiesbaden, 2006. MR 2327298 Zbl 1183.11023

[Böckle 2010] G. Böckle, "Deformation rings for some mod 3 Galois representations of the absolute Galois group of $\mathbf{Q}_{3}$ ”, pp. 529-542 in p-adic representations of p-adic groups, II: Representations of $\mathrm{GL}_{2}\left(\mathbb{Q}_{p}\right)$ and $(\phi, \Gamma)$-modules, edited by L. Berger et al., Astérisque 330, Société Mathématique de France, Paris, 2010. MR 2642411 Zbl 1223.11141

[Breuil 2003a] C. Breuil, "Sur quelques représentations modulaires et $p$-adiques de $\mathrm{GL}_{2}\left(\mathbb{Q}_{p}\right), \mathrm{I}$ ", Compositio Math. 138:2 (2003), 165-188. MR 2018825 Zbl 1044.11041

[Breuil 2003b] C. Breuil, "Sur quelques représentations modulaires et $p$-adiques de $\mathrm{GL}_{2}(\mathbb{Q} p)$, II", J. Inst. Math. Jussieu 2:1 (2003), 23-58. MR 1955206 Zbl 1165.11319

[Breuil and Emerton 2010] C. Breuil and M. Emerton, "Représentations p-adiques ordinaires de $\mathrm{GL}_{2}\left(\mathbb{Q}_{p}\right)$ et compatibilité local-global”, pp. 255-315 in p-adic representations of p-adic groups, III: 
Global and geometrical methods, edited by L. Berger et al., Astérisque 331, Société Mathématique de France, Paris, 2010. MR 2667890 Zbl 1251.11043

[Chenevier 2009] G. Chenevier, "Sur la variété des caractères $p$-adiques du groupe de Galois absolu de $\mathbb{Q}_{p}$ ", preprint, 2009, available at http://gaetan.chenevier.perso.math.cnrs.fr/articles/lieugalois.pdf.

[Chenevier 2014] G. Chenevier, "The $p$-adic analytic space of pseudocharacters of a profinite group and pseudorepresentations over arbitrary rings", pp. 221-285 in Automorphic forms and Galois representations (Durham, 2011), vol. 1, edited by P. K. F. Diamond and M. Kim, LMS Lecture Note Series 414, Cambridge University Press, 2014. Zbl 1310.11002

[Colmez 2010] P. Colmez, "Représentations de $\mathrm{GL}_{2}\left(\mathbb{Q}_{p}\right)$ et $(\phi, \Gamma)$-modules", pp. 281-509 in $p$-adic representations of $p$-adic groups, II: Representations of $\mathrm{GL}_{2}\left(\mathbb{Q}_{p}\right)$ and $(\phi, \Gamma)$-modules, edited by L. Berger et al., Astérisque 330, Société Mathématique de France, Paris, 2010. MR 2642409 Zbl 1218.11107

[Colmez et al. 2014] P. Colmez, G. Dospinescu, and V. Paškūnas, "The $p$-adic local Langlands correspondence for $\mathrm{GL}_{2}\left(\mathbb{Q}_{p}\right)$ )", Camb. J. Math. 2:1 (2014), 1-47. MR 3272011 Zbl 1312.11090

[Colmez et al. 2015] P. Colmez, G. Dospinescu, and V. Paškūnas, "Irreducible components of deformation spaces: wild 2-adic exercises”, Int. Math. Res. Not. 2015:14 (2015), 5333-5356. MR 3384443 Zbl 06513140

[Darmon et al. 1997] H. Darmon, F. Diamond, and R. Taylor, "Fermat's last theorem", pp. 2-140 in Elliptic curves, modular forms \& Fermat's last theorem (Hong Kong, 1993), edited by J. Coates and S. T. Yau, Int. Press, Cambridge, MA, 1997. MR 1605752

[Dospinescu 2015] G. Dospinescu, "Extensions de représentations de de Rham et vecteurs localement algébriques”, Compos. Math. 151:8 (2015), 1462-1498. MR 3383164 Zbl 06484396

[Emerton 2010a] M. Emerton, "Ordinary parts of admissible representations of $p$-adic reductive groups, I: Definition and first properties”, pp. 355-402 in p-adic representations of p-adic groups, III: Global and geometrical methods, edited by L. Berger et al., Astérisque 331, Société Mathématique de France, Paris, 2010. MR 2667882 Zbl 1205.22013

[Emerton 2010b] M. Emerton, "Ordinary parts of admissible representations of $p$-adic reductive groups, II: Derived functors”, pp. 403-459 in p-adic representations of p-adic groups, III: Global and geometrical methods, edited by L. Berger et al., Astérisque 331, Société Mathématique de France, Paris, 2010. MR 2667883 Zbl 1205.22014

[Emerton 2011] M. Emerton, "Local-global compatibility in the $p$-adic Langlands programme for $\mathrm{GL}_{2} / \mathbb{Q}$ ”, preprint, 2011, available at http://www.math.uchicago.edu/ emerton/pdffiles/lg.pdf.

[Emerton and Gee 2014] M. Emerton and T. Gee, "A geometric perspective on the Breuil-Mézard conjecture”, J. Inst. Math. Jussieu 13:1 (2014), 183-223. MR 3134019 Zbl 1318.11061

[Emerton and Paškūnas 2010] M. Emerton and V. Paškūnas, "On the effaceability of certain $\delta$-functors", pp. 461-469 in p-adic representations of p-adic groups, III: Global and geometrical methods, edited by L. Berger et al., Astérisque 331, Société Mathématique de France, Paris, 2010. MR 2667892 Zbl 1198.22010

[Fontaine 1994] J.-M. Fontaine, "Représentations p-adiques semi-stables", pp. 113-184 in Périodes p-adiques (Bures-sur-Yvette, 1988), Astérisque 223, Société Mathématique de France, Paris, 1994. MR 1293972 Zbl 0865.14009

[Gabriel 1962] P. Gabriel, "Des catégories abéliennes", Bull. Soc. Math. France 90 (1962), 323-448. MR 0232821 Zbl 0201.35602

[Gee 2011] T. Gee, “Automorphic lifts of prescribed types”, Math. Ann. 350:1 (2011), 107-144. MR 2785764 Zbl 1276.11085 
[Gee and Kisin 2014] T. Gee and M. Kisin, "The Breuil-Mézard conjecture for potentially BarsottiTate representations", Forum Math. Pi 2 (2014), art. ID e1. MR 3292675 Zbl 06391704

[Henniart 2002] G. Henniart, "Sur l'unicité des types pour GL2", (2002). Appendix to C. Breuil and A. Mézard, "Multiplicités modulaires et représentations de $\mathrm{GL}_{2}\left(\mathbb{Z}_{p}\right)$ et de $\operatorname{Gal}\left(\overline{\mathbb{Q}}_{p} / \mathbb{Q}_{p}\right)$ en $l=p$ ", Duke Math. J. 115:2 (2002), 205-310. Zbl 1042.11030

[Hu and Tan 2015] Y. Hu and F. Tan, "The Breuil-Mezard conjecture for non-scalar split residual representations", Ann. Sci. Éc. Norm. Supér. (4) 48:6 (2015), 1383-1421. MR 3429471 Zbl 1334.11041

[Katz and Messing 1974] N. M. Katz and W. Messing, "Some consequences of the Riemann hypothesis for varieties over finite fields", Invent. Math. 23 (1974), 73-77. MR 0332791 Zbl 0275.14011

[Khare and Wintenberger 2009a] C. Khare and J.-P. Wintenberger, "On Serre's conjecture for 2-dimensional mod $p$ representations of $\operatorname{Gal}(\overline{\mathbb{Q}} / \mathbb{Q})$ ”, Ann. of Math. (2) 169:1 (2009), 229-253. MR 2480604 Zbl 1196.11076

[Khare and Wintenberger 2009b] C. Khare and J.-P. Wintenberger, "Serre's modularity conjecture, II", Invent. Math. 178:3 (2009), 505-586. MR 2551764 Zbl 1304.11042

[Kisin 2008] M. Kisin, "Potentially semi-stable deformation rings", J. Amer. Math. Soc. 21:2 (2008), 513-546. MR 2373358 Zbl 1205.11060

[Kisin 2009a] M. Kisin, “The Fontaine-Mazur conjecture for GL2”, J. Amer. Math. Soc. 22:3 (2009), 641-690. MR 2505297 Zbl 1251.11045

[Kisin 2009b] M. Kisin, "Modularity of 2-adic Barsotti-Tate representations", Invent. Math. 178:3 (2009), 587-634. MR 2551765 Zbl 1304.11043

[Kisin 2009c] M. Kisin, "Moduli of finite flat group schemes, and modularity", Ann. of Math. (2) 170:3 (2009), 1085-1180. MR 2600871 Zbl 1201.14034

[Kisin 2010] M. Kisin, "Deformations of $\mathrm{G}_{\mathbb{Q}_{p}}$ and $\mathrm{GL}_{2}\left(\mathbb{Q}_{p}\right)$ representations", pp. 511-528 in p-adic representations of p-adic groups, II: Representations of $\mathrm{GL}_{2}\left(\mathbb{Q}_{p}\right)$ and $(\phi, \Gamma)$-modules, edited by L. Berger et al., Astérisque 330, Société Mathématique de France, Paris, 2010. MR 2642410 Zbl 1233.11126

[Paškūnas 2009] V. Paškūnas, “On some crystalline representations of $\mathrm{GL}_{2}\left(\mathbb{Q}_{p}\right)$ ", Algebra Number Theory 3:4 (2009), 411-421. MR 2525557 Zbl 1173.22015

[Paškūnas 2010a] V. Paškūnas, "Admissible unitary completions of locally $\mathbb{Q}_{p}$-rational representations of $\mathrm{GL}_{2}(F)$ ”, Represent. Theory 14 (2010), 324-354. MR 2608966 Zbl 1192.22009

[Paškūnas 2010b] V. Paškūnas, "Extensions for supersingular representations of $\mathrm{GL}_{2}\left(\mathbb{Q}_{p}\right)$ ), pp. 317-353 in p-adic representations of p-adic groups, III: Global and geometrical methods, edited by L. Berger et al., Astérisque 331, Société Mathématique de France, Paris, 2010. MR 2667891 Zbl 1204.22013

[Paškūnas 2013] V. Paškūnas, "The image of Colmez's Montreal functor”, Publ. Math. Inst. Hautes Études Sci. 118 (2013), 1-191. MR 3150248 Zbl 1297.22021

[Paškūnas 2014] V. Paškūnas, "Blocks for $\bmod p$ representations of $\mathrm{GL}_{2}\left(\mathbb{Q}_{p}\right)$ )", pp. 231-247 in Automorphic forms and Galois representations (94th London Mathematical Society (LMS) - EPSRC Durham symposium, UK, July 18-28, 2011), vol. 2, edited by P. K. F. Diamond and M. Kim, LMS Lecture Note Series 415, Cambridge University Press, 2014. Zbl 1310.11003

[Paškūnas 2015a] V. Paškūnas, “On 2-adic deformations”, preprint, 2015. arXiv 1509.00320

[Paškūnas 2015b] V. Paškūnas, “On the Breuil-Mézard conjecture”, Duke Math. J. 164:2 (2015), 297-359. MR 3306557 Zbl 06416950

[Saito 2009] T. Saito, "Hilbert modular forms and p-adic Hodge theory", Compos. Math. 145:5 (2009), 1081-1113. MR 2551990 Zbl 1259.11060 
[Schneider and Teitelbaum 2002] P. Schneider and J. Teitelbaum, "Banach space representations and Iwasawa theory”, Israel J. Math. 127 (2002), 359-380. MR 1900706 Zbl 1006.46053

[Serre 2000] J.-P. Serre, Local algebra, Springer, Berlin, 2000. MR 1771925 Zbl 0959.13010

[SGA 3 II 1970] M. Demazure and A. Grothendieck, Schémas en groupes, Tome II: Groupes de type multiplicatif, et structure des schémas en groupes généraux, Exposés VIII-XVIII (Séminaire de Géométrie Algébrique du Bois Marie 1962-1964), Lecture Notes in Math. 152, Springer, Berlin, 1970. MR 43 \#223b Zbl 0209.24201

[Taylor 2003] R. Taylor, “On icosahedral Artin representations, II”, Amer. J. Math. 125:3 (2003), 549-566. MR 1981033 Zbl 1031.11031

[Taylor 2006] R. Taylor, "On the meromorphic continuation of degree two L-functions", Doc. Math. Extra Vol. (2006), 729-779. MR 2290604 Zbl 1138.11051

[Thorne 2016] J. A. Thorne, "A 2-adic automorphy lifting theorem for unitary groups over CM fields", preprint, 2016, available at http://www.math.harvard.edu/ thorne/p_equals_2.pdf.

Communicated by Brian Conrad

Received 2015-09-01 Revised 2016-04-22 Accepted 2016-05-22

paskunas@uni-due.de

Universität Duisburg-Essen, Fakultät für Mathematik, Thea-Leymann-Str. 9, 45127 Essen, Germany 


\section{Algebra \& Number Theory}

msp.org/ant

\section{EDITORS}

MANAGING EDITOR

Bjorn Poonen

Massachusetts Institute of Technology

Cambridge, USA

\author{
EDITORIAL BOARD CHAIR \\ David Eisenbud \\ University of California \\ Berkeley, USA
}

BOARD OF EDITORS

$\begin{aligned} \text { Dave Benson } & \text { University of Aberdeen, Scotland } & \text { Susan Montgomery } & \text { University of Southern California, USA } \\ \text { Richard E. Borcherds } & \text { University of California, Berkeley, USA } & \text { Shigefumi Mori } & \text { RIMS, Kyoto University, Japan } \\ \text { John H. Coates } & \text { University of Cambridge, UK } & \text { Raman Parimala } & \text { Emory University, USA } \\ \text { J-L. Colliot-Thélène } & \text { CNRS, Université Paris-Sud, France } & \text { Jonathan Pila } & \text { University of Oxford, UK } \\ \text { Brian D. Conrad } & \text { Stanford University, USA } & \text { Anand Pillay } & \text { University of Notre Dame, USA } \\ \text { Hélène Esnault } & \text { Freie Universität Berlin, Germany } & \text { Victor Reiner } & \text { University of Minnesota, USA } \\ \text { Hubert Flenner } & \text { Ruhr-Universität, Germany } & \text { Peter Sarnak } & \text { Princeton University, USA } \\ \text { Sergey Fomin } & \text { University of Michigan, USA } & \text { Joseph H. Silverman } & \text { Brown University, USA } \\ \text { Edward Frenkel } & \text { University of California, Berkeley, USA } & \text { Michael Singer } & \text { North Carolina State University, USA } \\ \text { Andrew Granville } & \text { Université de Montréal, Canada } & \text { Vasudevan Srinivas } & \text { Tata Inst. of Fund. Research, India } \\ \text { Joseph Gubeladze } & \text { San Francisco State University, USA } & \text { J. Toby Stafford } & \text { University of Michigan, USA } \\ \text { Roger Heath-Brown } & \text { Oxford University, UK } & \text { Ravi Vakil } & \text { Stanford University, USA } \\ \text { Craig Huneke } & \text { University of Virginia, USA } & \text { Michel van den Bergh } & \text { Hasselt University, Belgium } \\ \text { Kiran S. Kedlaya } & \text { Univ. of California, San Diego, USA } & \text { Marie-France Vignéras } & \text { Université Paris VII, France } \\ \text { János Kollár } & \text { Princeton University, USA } & \text { Kei-Ichi Watanabe } & \text { Nihon University, Japan } \\ \text { Yuri Manin } & \text { Northwestern University, USA } & \text { Efim Zelmanov } & \text { University of California, San Diego, USA } \\ \text { Philippe Michel } & \text { École Polytechnique Fédérale de Lausanne } & \text { Shou-Wu Zhang } & \text { Princeton University, USA }\end{aligned}$

PRODUCTION

production@msp.org

Silvio Levy, Scientific Editor

See inside back cover or msp.org/ant for submission instructions.

The subscription price for 2016 is US $\$ 290$ /year for the electronic version, and $\$ 485 /$ year (+\$55, if shipping outside the US) for print and electronic. Subscriptions, requests for back issues and changes of subscribers address should be sent to MSP.

Algebra \& Number Theory (ISSN 1944-7833 electronic, 1937-0652 printed) at Mathematical Sciences Publishers, 798 Evans Hall \#3840, c/o University of California, Berkeley, CA 94720-3840 is published continuously online. Periodical rate postage paid at Berkeley, CA 94704, and additional mailing offices.

ANT peer review and production are managed by EditFLow ${ }^{\circledR}$ from MSP.

\section{PUBLISHED BY}

- mathematical sciences publishers

nonprofit scientific publishing

http://msp.org/

() 2016 Mathematical Sciences Publishers 


\section{Algebra \& Number Theory}

Volume $10 \quad$ No. $6 \quad 2016$

Modular elliptic curves over real abelian fields and the generalized Fermat equation $x^{2 \ell}+y^{2 m}=z^{p}$

SAMUELE ANNI and SAMIR SIKSEK

Geometry and stability of tautological bundles on Hilbert schemes of points

DAVID STAPLETON

Anabelian geometry and descent obstructions on moduli spaces

Stefan PATRIKIS, José Felipe Voloch and Yuri G. Zarhin

On the local Tamagawa number conjecture for Tate motives over tamely ramified fields JAY DAIGLE and MATTHIAS FLACH

Heegner divisors in generalized Jacobians and traces of singular moduli

JAN HENDRIK BRUINIER and YINGKUN LI

On 2-dimensional 2-adic Galois representations of local and global fields VYTAUTAS PAŠKŪNAS

A probabilistic Tits alternative and probabilistic identities

Michael LARSEN and ANER SHALEV 